\title{
ENSEMBLE CHARACTERISTICS OF VISUAL EVOKED CORTICAL POTENTIALS IN NOISE
}

By

TIMOTHY C. DOYLE

\begin{abstract}
A DISSERTATION PRESENTED TO THE GRAdUATE COUNCIL OF
THE UNIVERSITY OF FLORIDA

IN PARTIAL FULFILLMENT OF THE REQUIREMENTS FOR THE DEGREE OF DOCTOR OF PHILOSOPHY
\end{abstract}

UNIVERSITY OF FLORIDA

1969 


\section{ACKNOILEDGMENTS}

The author wishes to express his sincere appreciation to Dr. D.G. Childers, chairman of his supervisory comnittee, for his counse 1 and encouragement. He also wishes to thank Dr. N.W. Perry and Dr. J.R. Smith, the other members of his supervisory cominittee, and Dr. A.H. Nevis for their guidance. He would like to acknowledge the Visual Sciences Laboratory and staff for their facilities and help. 


\section{CHAPTER}

1 INTRODUCTION . . . . . . . . . . . . . . . . 1

2 SEQUENTIAL AND ENSERBLE AVERAGING ........... 4

2.1 Sequential Averaging ........... 47

2.2 Variability of the VER .......... 10

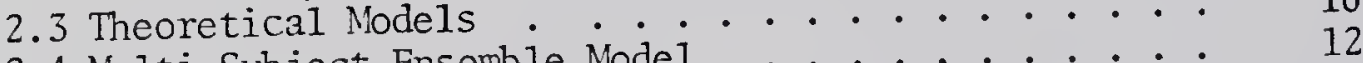

2.4 Multi-Subject Ensemble Model ............... 13

2.5 Ensemble Averaging . . . . . . . . . . 15

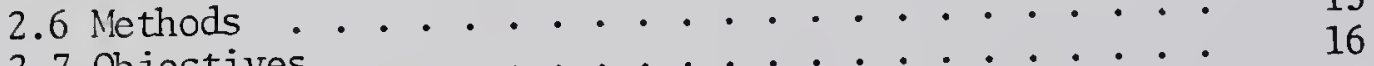

2.7 Objectives . . . . . . . . . . . . . . . . . .

3 RESULTS OF FNSEMBLE AVERAGING . . . . . . . . 19

3.1 Introduction .................. 19

3.2 Selection of Bandwidth . . . . . . . . . 21

3.3 Ensemble Evoked Response ............ 22

3.4 Noise in the Ensemble Evoked Response . . . . . . 28

3.5 Cuality of the Ensemble Evoked Response Estimate . • 32

3.6 Variations in the Ensemble Evoked Response with

Stimulus Number . . . . . . . . . . 36

3.7 Average of Ensemble Evoked Responses . . . . . . 39

3.8 Surnary . . . . . . . . . . . . . . 41

4 THEORETICAI ASSUMPTIONS UNDERLYING RESPONSE AVERAGLNG .. 43

4. I Stationarity of the Background Noise . . . . . . . 44

4.2 Dependence of Signal and Noise . . . . . . . 56

4.3 Additivity of Signal and Noise . . . . . . . 59

4.4 Sumary ........................ 67

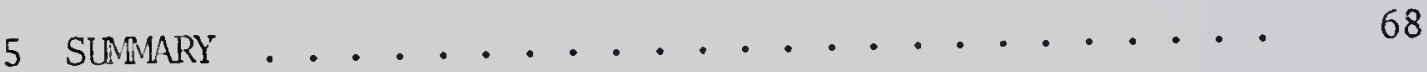


APPENDIX

A EXPERIMENTAL PROCEDURE . . . . . . . . . . . . 75

B SUPPLEMENTARY FIGURES AND TABLES . . . . . . . . 78

REFERENCES ............................... 92

BIOGRAPHICAL SKETCH ................... 97 
3.1 Average Correlations for Figure 3.2 . . . . . . 25

3.2 Average Correlations for Figure 3.4 . . . . . . 28

4.1 Maximum Number of Standard Deviations of Ensemble Control Average about a Zero Mean ........... . 47

4.2 Maximum Number of Standard Deviations of \pm Estimate about a Zero Mean ............. 48

4.3 Maximum Number of Standard Deviations (times 0.1 ) of EER Estimate about Zero Mean . . . . . . . . 49

4.4 RMS Values in $\mu v$ of \pm Averages Under Stimulus and Non-Stimulus Conditions...........

B.1

through

B.7 Response Correlations Coefficients . . . . . . . 88

B. 8

through

B.13 EER Correlation Coefficients for the 0.3 to $50 \mathrm{~Hz}$

Bandwidth ............. . . 89

B. 14

through

B.20 Control Correlation Coefficients ......... 90

B.21 Values for $\tilde{C}_{f}(t, t-\tau)$ in Figure $4.1 \ldots 91$ 


\section{LIST OF FIGURES}

2.1 Ensemble Versus Sequential Averaging .......... 14

3.1 Bandwidth Comparison .............. 21

3.2 Ensemble Evoked Response . . . . . . . . . . . 23

3.3 Superimposed Ensemble Evoked Response . . . . . . . . 27

3.4 Ensemble Control Averages . . . . . . . . . . 29

3.5 Noise Amplitude in EER . . . . . . . . . . . . 31

3.6 Superimposed Ensemble Standard Deviations . . . . . . . 32

3.7 EER Confidence Intervals . . . . . . . . . . . 34

3.8 VER Confidence Intervals . . . . . . . . . . . 35

3.9 Amplitude Fluctuations of the Primary Response . . . . . 38

3.10 Latency Fluctuations of the Three Primary Components . . . 38

3.11 Average of Response and Control Ensemble Averages . . . 40

4.1 Ensemble Autocovariance Function for First Control . . 51

4.2 Superimposed Ensemble Standard Deviations . . . . . . 53

4.3 Histogram Estimates of Ensemble pdf for First Control . . 55

4.4 S/N Ratio Versus Number of Summations . . . . . . . . 65

B.1 EER for Two Bandwidths . . . . . . . . . . . 79

B.2 Ensemble Control Average for Two Bandwidths . . . . . 80

B.3 \pm Ensemble Responses for Two Bandwidths . . . . . . 81

B.4 4 Ensemble Control Averages for Two Bandwidths . . . . 82

B.5 Ensemble Standard Deviations Under Stimulus and Non-Stimulus Conditions . . . . . . . . . 83

B.6 Ensemble Autocovariance Function for First Response . . 84 
B.7 Ensemble Autocovariance Function for Fourth Response. . . 85

B. 8 Histogram Estimates of Ensemble pdf for First Response . . 86

B.9 S/N Ratio Versus Number of Sumations . . . . . . . . 87 
Abstract of Dissertation Presented to the Graduate Council in Partial Fulfillment of the Requirements for the Degree of Doctor of Philosopiny

ENSEMBLE CHARACTERISTICS OF VISUAL EVORED CORTICAL POTENTIALS IN NOISE

By

$$
\begin{gathered}
\text { Timothy C. Doyle } \\
\text { August, } 1969
\end{gathered}
$$

Chairman: Dr. D.G. Childers

Major Department: Electrical Engineering

To date, most investigations of evoked potentials monitored at the human scalp have utilized the average of a series of responses recorded from a single subject to define components of the evoked response, ER. This technique has provided a fairly consistent estimate of an individual's average ER. However, with this method it is not uncommon to obtain quite dissimilar averages from different subjects under identical experimental conditions. This contrast in the ER estimates across a population has hindered the interpretation of the effects of stimulus parameters on the ER and frustrated the attempt to use this average for clinical diagnosis.

The present study investigated one method of obtaining information about the character of the response to visual stimuli common to a considered normal subject population. The estimation technique utilized was to average across an ensemble of fifty subject EEG's containing evoked activity. This method permitted estimation of common inter-subject response activity evoked by a single stimulus. 
By averaging the resultant waveforms of this ensemble average across stimuli, a reliable, relatively noise-free inter-subject response template was obtained.

The resemblance of the ensemble of cortical potentials to a classical stochastic process enabled the author to investigate three prevalent assumptions about the statistical character of the evoked response and background noise activity. These three assumptions are: (1) the response and background noise are independent; (2) the background noise is stationary; and (3) the response and noise are additive. The results presented in this dissertation lend some measure of support to each of the three assumptions. 
CHAPTER 1

INTRODUCTION

The time-varying electroencephalographic potential specifically elicited by a single controlled sensory stimulus is referred to as an evoked potential or evoked response (ER). An invariant evoked response to controllable stimulus parameters, monitored from the human scalp, would provide an excellent empirical diagnostic tool for brain research. However, this has eluded all investigators because of two inherent limitations. First, the parameters affecting the ER, such as physiological variability of the subject during stimulation, are usually unknown and uncontrollable. Second, the observability of a single response is severely handicapped by the interaction of the desired signal (ER) with the background bioelectric on-going activity.

The physiological variability, or state of the subject, introduces a random variable into what might otherwise have been a deterministic response. The on-going background activity masks and distorts the ER such that it usually is undetectable at the scalp. Thus, these two sources of variability deny the investigator anything but tenuous conclusions about the relationship of a single bioelectric response to specific stimulus parameters. In order to determine the general characteristics of an ER, several data processing techniques have been utilized. The most common technique is the sequential 
average (see Chapter 2) of a series of responses recorded from an individual subject. This average is fairly consistent for a particular subject under identical stimulus conditions. However, the averages obtained from several subjects often appear to be quite dissimilar. Thus, it is difficult to establish a certain average response waveform, elicited by specific stimulus parameters across a subject population.

In the present study the average of a single response across a subject population is investigated. This average provides an estimate of the characteristics of the response to a particular stimulus which are conmon to an ensemble of subjects. From these common characteristics one can develop a model or template of the average response activity across the ensemble. The changes in this response average with repetitions of a specific stimulus can also be investigated. In addition, the collection of subject EEG waveforms can be viewed as an ensemble of independent sample functions from a stochastic process. Thus, assumptions underlying the averaging process can be examined by classical techniques. These assumptions generally involve the statistical nature of the evoked signal and background noise.

Therefore, the two major objectives of this investigation are: (1) to develop a better understanding of the common characteristics or norm of the evoked responses within a subject population; and (2) to provide an empirical technique to evaluate the prevalent statistical assumptions about the character of the evoked response and background potentials. Since at this time the response signal cannot be separated from the background noise, the results are 
limited to some combination of the signal and noise rather than either by itself. The characteristics of the noise under non-stimulus conditions are observed, but these may provide little insight into the background noise activity during stimulation. Therefore, the second objective is theoretically unattainable at the outset. However, the results obtained in this study should contribute to the knowledge of the statistical character of evoked on-going activity.

The results of this investigation are divided into two chapters. Chapter 3 explores the inter-subject norm or ensemble average and the transient changes in this average. Also examined are the non-stimulus control estimates of the average background activity. Chapter 4 contains an examination of three assumptions about the signal and noise which are necessary to theoretically justify results obtained by these averaging techniques. In this examination the statistical character of the ensemble of ER's with background noise and the ensemble of resting bioelectrical potentials is investigated. To facilitate understanding of the results presented in these chapters, Chapter 2 provides an overview of the background material and methodology for this investigation. 


\section{SEQUENTIAL AND ENSEMBLE AVERAGING}

The peak-to-peak amplitude of the potentials recorded from the scalp usually have a range of 9 to $50 \mu \mathrm{v}$ (Cobb, 1963). In most cases the amplitude of the evoked activity in these potentials is below the threshold of observability. It therefore becomes necessary for the researcher to preprocess the scalp potentials to extract some characteristics of the ER. Generally, these processing techniques require reiterative measures and estimate the response characteristics in terms of probability statements or statistical averages.

\subsection{Sequential Averaging}

To date the most powerful statistical descriptor of the ER is the sequential average which was implemented photographically by Galambos and Davis (1943) and later electromechanically by Dawson (1954) and electronically by Barlow (1957). Today this average is usually calculated by a general or special purpose digital computer. The sequential average is essentially the cumulativs sum or average of successive trains of the scalp potentials which attend a series of repeated identical stimuli. The mathematical expression for this average is

$$
\tilde{m}_{i}(t)=\frac{1}{N} \sum_{j=0}^{N-1} f_{i}(t+j T) \quad \text { for } 0 \leqq t \leqq T
$$

where $f_{i}(t)$ is the time varying EEG (electroencephalogram) recorded from subject $i$ and the stimulus cccurs periodically $N$ times at $t=0, T$, $2 \mathrm{~T} . . . T_{\text {The }}$ indicates the above is an estimate of the true mean 
$m(t)$ for the range of $t$ between 0 and $T$. If the stimuli are visual, the result of this average is called a visual evoked response (VER). Since the present study employs visual stimuli, most of the discussion will focus on responses evoked in this modality.

The basic advantage of sequential averaging over other data processing methods is that it extracts an estimate (e.g., the VER) of the response waveshape from the random potential fluctuations monitored at the scalp. Other data processing techniques such as autocorrelation (Barlow, 1959), spectral analysis (Childers, et al., 1968), matched filtering (Negin, 1968), and synchronous correlation (Regan, 1966a) may detect the periodicity, frequency composition, presence, or phase locked components of the ER, respectively, but they do not produce the waveshape information provided by averaging. In addition, the apparent underlying integration mechanisms noted in most complex neurological systems tend to justify or lend a degree of rationale to the averaging technique, as Rosenblith (1959, p. 540) reasons:

In the handling of sensory information, organisms behave as if they were acting on the basis of activity averaged over substantial regions of the nervous system, such a weighted averaging process could be carried out by comparing the outputs of a large number of neural elements. Since there are at present no devices of performing this task (even if one knew exactly where and how to perform it), one chooses to average over an ensemble of responses to repeated identical stimuli in order to bring out certain typical aspects of behavior of the nervous system.

The major disadvantages of sequential areraging are: (1) only those components of the ER which are time locked to the stimulus will be extracted; (2) the responses to succeeding stimuli may differ and distort the average response estimate because of the effects of those 
preceding them; and (3) the resultant average of many responses is not identical under invariant stimulus conditions. By extracting only the time locked components, the averaging process obscures any phase and frequency deviations of the individual responses. In addition, the degree of amplitude variation of these time locked components is hidden within the resultant waveform. However, it is impossible to improve the averaging process by weighting and phase locking each response without the a priori knowledge of the specific angle or amplitude modulation which may affect a series of responses. Recent studies by Harris and Woody (1969) and Woody (1967) have attempted to estimate the degree of angle modulation of the ER by a correlation technique. From this estimate, they have suggested an adaptive filter to improve signal extraction by averaging. However, to most investigators such modulation is a perturbing factor distorting the estimate obtained by averaging.

The second disadvantage is imposed by the practical limitation that the large number of stimuli necessary to extract an average response is generally presented over a relatively short period of time. This limitation introduces two disturbing factors. The first factor can be termed overlap interference, i.e., the transient tail of one response overlaps and distorts the initial components of the subsequent response. This usually occurs only with relatively high stimulus rates. The critical stimulus frequency at which this interaction occurs is not clearly established, but the concensus of evidence indicates that it lies in the region of $0.7 \mathrm{~Hz}$ (Barlow, 1960) to $2.7 \mathrm{~Hz}$ (Kitzsato, 1966). There is some indication that higher rates 
are permissible if the stimulus is presented at random intervals (Ruchkin, 1965).

The second disturbing factor is habituation, which can generally be described as the decrease in the subject's attention to monotonous repetitive stimulation, which changes the successive responses. Since the extraction by averaging of a reliable ER requires many repetitions of the stimulus (see Perry and Childers, 1969) most investigators discuss habituation in terms of the changes in consecutive averages of large groups of successive responses. Therefore short term habituation, particularly transient changes of the ER's to the initial stimuli, is difficult to record. However, Zerlin and Davis (1967) utilizing a highly responsive subject, have noted a systematic downward drift in the amplitude of individual responses to successive auditory stimuli. This result agrees with the less specific gradual waxing and waning decrease in amplitude of consecutive averages (VER's) found by many authors (e.s., Perry and Copenhaver, 1965; Haider, et al., 1954; and Garcia-Austt, et a1., 1963). Bogacz, et al. (1960) have also noted that habituation to visual stimuli is also reflected in increased phase lag (latency) of certain averaged response components.

\subsection{Variability of the VER}

The third shortcoming of sequential averaging, the variability of the resultant, poses the greatest dilenma to the researcher using this tool. The VER's taken on different occasions from a single subject are relatively stable over a period of time (Dustman and Beck, 1965); however, when one compares the VER's cbtained under jdentical stimulus conditions from a population of subjects, variability of the waveform 
makes it difficult to interpret the effect of certain stimulus parameters on the human visual system. Some investigators have attempted to recuce this inter-subject variability by expanding the resultant waveform into orthogonal sets for comparison, with little success (see Donchin, 1966 and John, et al., 1964). Others have organized a system of classification of the certain maxima and minina of the VER waveform in terms of amplitudes and time delays (latencies) from stimulus onset (see Bergamini and Bergamasco, 1967 and Kooi and Bagchi, 1964). With this latter technique, investigators have been able to occasionally detect trends of change common to a subject population with gross variations in the stimulus parameters. But such findings are again hampered severely by the variability of the VER. This variability appears to be the product of changes in the psychological state of the subject (e.g., attention) and the inherent statistical character of the ER and background activity (Werre and Smith, 1964).

In an attempt to understand the statistical properties of the ER and background noise, a vast amount of effort has been concentrated on the classification of the physiological origin of these scalp potentials. However, to date there is only a rudimentary knowledge of the mechanisms which underlie the generation of these EEG waveforms (Elul, 1967). The present evidence indicates that EEG potentials arise from some complex integration of excitatory and inhibitory post synaptic potentials, psp (Humphrey, 1968; Creutzfeldt and Kuhnt, 1967; Eccles, 1966 and Amassian, et al., 1964). The reiationship between these subcranial potentials and the bioelectric waveform monitored at the scalp has been investigated in animals by De Jucchi, 
et al. (1962). They have hypothesized that the scalp acts as a further averager of psp's. In similar investigations involving human subjects, Cooper, et al. (1965) demonstrate that only an average of those potentials which are widely synchronized can be observed in the scalp. It is therefore interesting, as Rosenblith (1959) notes, that even with this gross averaging of psp's by the scalp electrodes, we still find variability of the ER to identical stimuli.

The amount of this variability produced by the interaction of the response signal with the background activity (noise) is generally unknown. To date, most investigations of this interaction have concentrated on only one component of the noise (resting EEG), the alpha rhythn. This component is defined by Brazier, et al. (1961, p. 647) as the "rhythm, usually with frequency of $8-1.3 \mathrm{c} / \mathrm{sec}$, of almost sinusoidal form, in the posterior areas, present during relaxation when the eyes are closed, attenuated during attention, particularly visual." The physiological origin of this regular rhythmic activity is yet undetermined. However, Andersen and Andersson (1968) point to the thalamus as the source of the rhythmic impulses which trigger the psp's monitored at the scalp. This agrees with the observations of Creutzfeldt, et al. (1966). The alpha rhythm is the most striking component of the resting EEG of the majority of normal adulis. This compcnent is so prevalent that a resonant peak around $10 \mathrm{~Hz}$ can readily be seen in the EEG frequency spectrum (Walter, et a!., 1967). In addition, the late rhythmic component (after discharge) of the ER and VER has the general form and dimensions of the spcntareous alphu wave (Bishop and Clare, 1953 and Barlow, 1950). It is therefore not mexpected that studies involving the relatjonsinip 
of this predominant EEG component to evoked responses, particularly those elicited by visual stimuli, received precedence.

A review of the studies focusing on this interaction with the VER is provided in the recent work by Childers and Perry (1969). They note that many investigators suspect two forms of 8 to $13 \mathrm{~Hz}$. activity: the first, the predominant non-provoked EEG activity, and the second, the components of the evoked response which are alphalike in character. The evidence presented indicates that if two distinct forms of activity exist, they probably share common neural ensembles. However, this relationship is unclear and Childers and Perry stress the need for further experimentation to secure the additional information necessary to determine the interaction of these components and other background activity with the ER.

\subsection{Theoretical Mode1s}

Several investigators (e.g., Ruchkin, 1969; Kitasato and Hatsuda, 1965; Bendat, 1964; and Goldstein and Weiss, 1962) attempt to understand the mechanisms of the VER and hence its variability by developing theoretical models of the statistical character of scalp potentials. These studies generally rely on the hypothesis that the signal (ER) and noise (background activity) are additive. This assumption permits a certain amount of mathematical tractability to these models but contains little physiological basis. However, it is justified in part because of the ability to extract a somewhat reproducible estimate of the signal from the scalp potentials by sequential averaging. For example, if the signal and noise were multiplicative, this 
extraction technique would provide conpletely inconsistent results.

In order to facilitate statistical analysis of the scalp potentials, these model developers generate an artificial stochastic process. This process is constructed by segmenting (usually at the points of stimulation) the provoked EEG record into an ensemble of shorter records. The statistical moments of this ensemble can then be calculated from the empirical data and compared to the theoretical model (e.g., the first moment is the sequential average). Unfortunately, few authors have progressed past the modeling stage to compare the theory with practice. This gap exists, because, as Barlow (1967) notes, the theoretical ability to separate the statistical components (i.e., signal and noise) does not have an experimental counterpart. Such a separation would require a much greater knowledge of the character of either the signal and/or noise and their interdependence than is available at the present.

In addition, the assumptions made about the stochastic model are generally violated in the empirical ensemble. For example, the empirical statistics are derived from temporally related members, which requires a stationary ergodic random process. This is highly improbable if one notes the existence of a time varying mean (the VER signal). Also the members would probably have some statistical dependence, reducing the quality of the empirical statistics. Finally, each subject would have to be examined separately, which would eliminate the estimation of the inter-subject statistics. However, is there a better stochastic model and is such a model feasible? This question initiated the present study and led to the 
development of the following model of a multi-subject random process.

\subsection{Multi-Subject Ensemble Mode1}

It was decided that a more appropriate empirical stochastic process might be generated from an ensemble of individual EEG's (i.e., each EEG which contains evoked activity is recorded from a different subject). Such an ensemble would eliminate the temporal and probably the statistical dependence of its members. Thus, this ensemble would more accurately comply with the classical definition of a stochastic process as a process composed of an ensemble of independent simultaneously monitored sample functions. The empirical ensemble statistics would then provide a more precise evaluation of the statistical assumptions necessary to theoretically justify averaging to extract a consistent response estimate. In addition, these ensemble statistics, particularly the first moment (average) of the ensemble, would provide information about common inter-subject characteristics of the ER.

The inherent limitations of this artificial process are two-fold. First, the individual records are not simultaneously monitored, but serially recorded over a several month period. Second, the evoked activity in the member functions may result from several stochastic processes instead of simply one. The first restriction becomes significant if the nature of a normal adult EEG is dependent upon the day of the year or the hour of the day. Fortunately, however, the present evidence points to a relatively stable individual EEG, particularly in frequency composition (Berkhout and valter, 1968 and 
Johnson and Ulett, 1959). The second limitation becomes important if the population of ER's can be divided into discrete classes. To date, there has been some indication (Perry, et al., 1968) that such sub-divisions might exist; however, the method of classification and the number of classes is yet undetermined. Therefore, the present research effort will assume a continuum of EEG and response types which can be modeled under the same stochastic process.

\subsection{Ensemble Averaging}

The ensemble mean or average provides an estimate of the common inter-subject response to a single stimulus. The mathematical expression for this average is

$$
\tilde{m}_{f}(t)=\frac{1}{N} \sum_{i=1}^{N} f_{i}(t)
$$

where $f_{i}(t)$ is the EEG containing evoked activity recorded from subject $i$ out of a population of $N$ subjects. This ensemble average, VER, and unprocessed scalp potentials are schematically represented in Figure 2.1. This figure demonstrates the basic difference in the ensemble and sequential average (i.e., VER). The ensemble average provides an estimate of the inter-subject average respense to a single stimulus and the VER provides the inter-stimulus average response for a single subject. The ensemble average eliminates the inter-subject response variability while it reflects the changes in the average response to a series of individual stimuli. The VER average eliminates the inter-stimulus response variability while it reflects the 


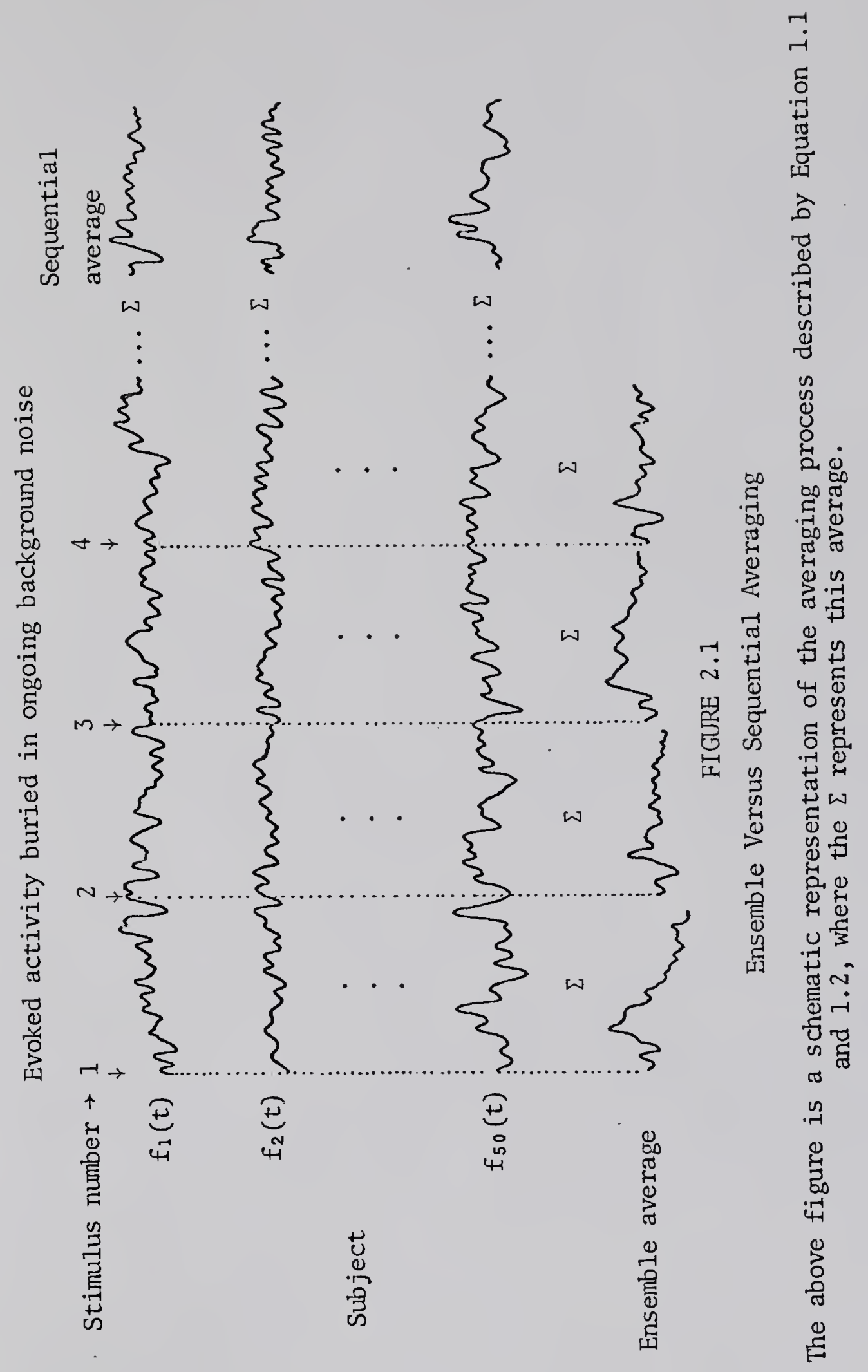


individuality of a subject's average response.

The average of a set of VER's from a subject ensemble equals the average of the set of ensemble averages for a series of stimuli, provided both averages are obtained from the sane inclusive collection of data. This double average provides an overall estimate of the response activity by sacrificing all variability information. This overall average has been suggested by Dawson, et al. (1968) to reduce all excess information about the response. Therefore, this single estimate might provide a general inter-subject, inter-stimulus response norm to specific stinulus parameters.

\subsection{Methods}

In the initial stages of this investigation it was decided that an ensemble size (subject population) of fifty would provide reasonable statistical accuracy and remain within the realm of experimental practicability. Both stimulus (signal) and non-stimulus (noise) trials were recorded from each of the subjects. The stimulus was a monocular periodic flash of light which had a repetition rate of about one flash per second. The stimulus was visually occluded during noise (control) trials. The potentials recorded in these trials were monitored from a pair of scalp electrodes vertically oriented on the occiput midline. These potentials were amplified by a factor of $100 \mathrm{~K}$; separated into two data channels (i.e., a wide band, 0.3 to $50 \mathrm{~Hz}$, channel and a narrow band, 0.3 to $15 \mathrm{~Hz}$, channe1); and recorded on magnetic tape.

These recorded potentials were then digitized and stored in an 
IBM 1800 computer in order to facilitate necessary mathematical computation. Computer memory limitations restricted the amount of data analyzed. Therefore, most of the results presented in this study are derived from activity following ten stimulus triggers (both signal and noise trials) selected from the periodic (approximately $1 \mathrm{~Hz}$ ) stimulus train. The triggers chosen are the first through fifth, tenth, twentieth, thirtieth, fortieth and fiftieth in the repetitive stimulus train. All of the computation was performed on the first 950 milliseconds of data following each trigger. A more detailed discussion of the data collection and analysis procedure is provided in Appendix A.

\subsection{Objectives}

The two objectives of this study, as stated in the introductory chapter, are: (1) to develop a better understanding of the common characteristics or norm of the evoked responses within a subject population and (2) to provide an empirical technique to evaluate the prevalent statistical assumptions about the character of the ER and background potentials. The inter-subject ensembles recorded under stimulus and non-stimulus conditions provide data from which both these objectives can be pursued. The average across the ensemble under stimulus conditions furnishes an estimate of the common intersubject evoked activity. The data provided by these ensembles is appropriate for the investigation of theoretical assumptions about the EEG stochastic process since these potentials are a reasonable facsimile of such a process. 
In pursuit of the first objective the ensemble average across fifty subjects under stimulus conditions is examined. It is anticipated that the results from this average will reveal the components of the evoked activity which are the most consistent across the subject population. These components should reappear after each stimulus with some degree of variability. The variability would probably be the result of transient changes (e.g., habituation) in the inter-subject response with the number of stimuli, variations introduced by the inherent error of the estimation process, or background noise potentials which may remain in the average. A transient variation should result in some overall trend of change in the response average while noise interference and estimation error should affect the average in a more random manner. The transient trends of response estimate and confidence in this estimate across a series of stimuli will be investigated. The ensemble average under non-stimulus conditions is expected to provide an approximation of the amount of noise contained in the response estimate. The average of a collection of ensemble averages should eliminate transient variability, reduce the components of the background noise and provide a fairly stable inter-subject response tenplate.

In order to theoretically justify the above response estimation by averaging, it is necessary that (1) the background noise come from a relatively wide serise stationary random process; (2) the signal and noise processes be statistically independent; and (3) the signal and noise be additive. Since the signal and noise potentials cannot be 
separated at the present time, the above properties are generally assumed to be true. The second objective of this study, evaluation of these assumptions, is hindered by the inability to segregate the signal and noise. However, by hypothesizing one or two of these assumptions an attempt can be made to empirically assess the third. For example, by hypothesizing the signal and noise to be statistically independent, the inter-subject ensemble under non-stimulus conditions should approximate the noise activity under stimulus conditions. The stationarity (i.e., time dependence of the ensemble statistics) of the background noise can then be examined using the resting EEG as noise approximation. Evaluation of the second assumption can be obtained in a similar manner by hypothesizing the third assumption and examining the \pm ensemble average and ensemble variance under stimulus and non-stimulus conditions. The assessment of the third assumption is more difficult. However, by hypothesizing the first two assumptions we can examine the growth of the signal to noise $(\mathrm{S} / \mathrm{N})$ ratio, speculating the signal to be the ensemble average and the noise the I ensemble average under stimulus conditions. If the signal and noise are additive, the $\mathrm{S} / \mathrm{N}$ ratio should grow linearly with the nunber of averages.

The results presented in the two succeeding chapters attempt to realize the above objectives. The response activity common to the subject ensemble is first examined, followed by the evaluation of the statistical assumptions about the character of the ER and background noise. 
CHAPTER 3

RESULTS OF ENSEMBLE AVERAGING

\subsection{Introduction}

In this chapter the inter-subject average response to a monocular visual stimulus is examined. The average across the fifty subject ensemble record (see Figure 2.1) is utilized to extract an estimate of the response activity from the random scalp potentials. This ensemble average should provide an inter-subject response norm if: (1) the assumptions mentioned in the previous chapter hold; (2) there exist stimulus locked response components which are common to the subject population; and (3) the amplitude of the average background activity in this estimate is much less than the amplitude of these response components. The first condition, the validity of assumptions about the response signal and background noise necessary to justify averaging, is the subject of Chapter 4. The present chapter will hypothesize these assumptions to be true and exanine the ensemble average for any inter-subject stimulus locked response components. In addition, an estimate of the amount of background noise contained in this average is investigated.

In order to simplify discussion of the ensemble average evoked response estimate the term ensemble evoked response (EER) will be utilized. This term is analogous to the term visual evoked response 
(VER) in that it is not intended to imply that the average response estimate contains only evoked activity. Theoretically the VER and EER contain noise components whose variance decreases directly with the number of sums in the average (see Perry and Childers, 1969). An estimate of the amount of noise in the EER is obtained from the average across the ensemble under non-stimulus conditions. This average provides a series of control waveforms, each attending a particular occluded stimuli, whose amplitudes should provide an estimate of the background noise in the EER. This background noise activity which is not related to the stimulus will introduce variations in the EER from one stimulus to the next.

As noted in the previous chapter, other parameters which could affect the EER across stimuli are deviations of the estimate about the true mean and variations in this response mean with stimulus number. The deviation of the EER from the true mean is the result of the variance of the potentials across the ensemble (see Bendat and Piersol, 1966). Both the response signal and background noise contribute to this variance since it is unlikely that the response activity from every subject is identical and the noise is random. However, since the signal activity generally has less amplitude (Perry and Childers, 1969) and greater similarity across the ensemble than the noise potential, the latter should account for most of the ensemble variance. Therefore the estimation deviations of the EER should be primarily the result of the background noise. The range of these deviations can be predicted utilizing the Tchebycheff inequality and 
ensemble variance. This prediction takes the form of confidence intervals about the EER.

In order to examine the second parameter, the variations in the EER caused by changes in the actual inter-subject response, it is necessary to separate response signal from the noise activity in the ensemble average. Since this is not possible at this time, the present investigation will generally be restricted to variations in the more prominent components of the EER's which are fairly consistent across stimuli. Since the stimuli are identical, these variations should result in some trend of change rather than random fluctuations.

\subsection{Selection of Eandwidth}

The EER and other results presented in this chapter and the succeeding chapter are obtained from the stimulus provoked and resting EEG ensembles mentioned in Chapter 2. In addition, most of the discussion of these results is limited to narrow band filtered 0.3 to $15 \mathrm{~Hz}$ ) potentials. Although the fidelity of this more restrictive

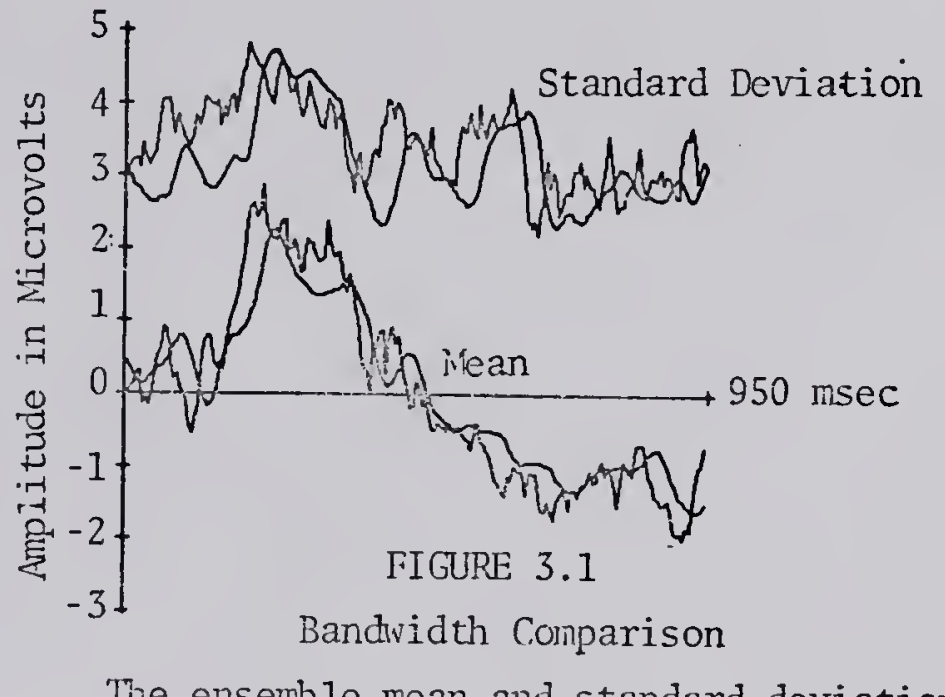

The ensemble mean and standard deviation for two bandwidths are superimposed. 
bandwidth is not as good as the wider band potentials $(0.3$ to $50 \mathrm{~Hz})$, it still retains the significant EEG potential fluctuations, as Figure 3.1 demonstrates. In this figure the first and second ensemble moments for both bandwidths are superimposed and reflect only minor variations, including a slight phase shift introduced by the filter. In addition, the upper limit of $15 \mathrm{~Hz}$ exceeds the minimum range of ten times the frequency of stimulation suggested by Childers, et al. (1968) to recover a VER average with good fidelity. Therefore, only the narrow band averages which provide an accurate description of the EEG activity will usually be discussed. However, many of the results presented here are duplicated for the wider band potentials in Appendix B. This appendix also contains other results which are not pertinent to the discussion. Any reference to these results will have a prefix $B$.

\subsection{Ensemble Evoked Response}

The ensemble evoked response, EER, to the selected photic stimuli is provided in Figure 3.2. Each average is designated by the number signifying which stimulus within the train evoked it. In each waveform the flash stimulus occurs at the extreme left of each 950 millisecond response average. The first four ensemble response averages can also be found in Figure 2.1, which depicts the method by which the averages were obtained. The waveforms are segmented into seven 135 millisecond intervals to facilitate comparison and discussion. Again, it should be pointed out that each EER is only an estinate of the actual evoked potential and contains both evoked and background activity. 
Interval

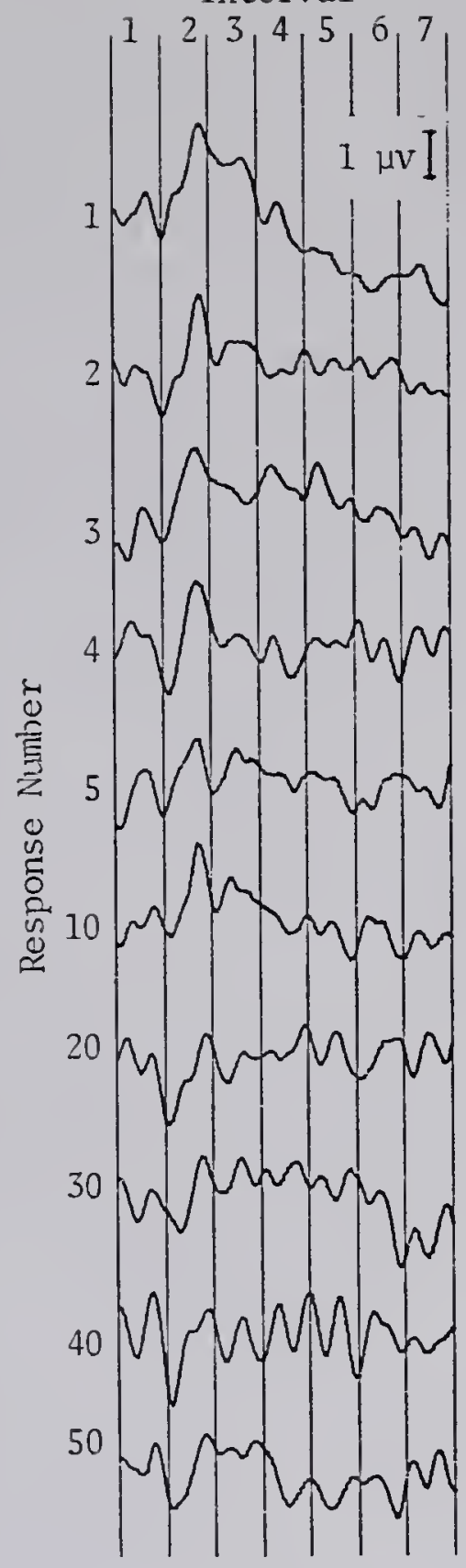

FIGURE 3.2

Ensemble Evoked Response

Ensemble averages for ten selected stimuli are divided into 7 intervals of 135 miliiseconds.
A cursory glance at the EER's in Figure 3.2 provides the reader with little indication that the fluctuating waveforms contain any response activity. However, if one utilizes the inverted sinusoid in the second interval of the fourth response as an ER template, the most common attribute of the waveform set becomes more apparent. This inverted sinusoid, although sometimes partially distorted, appears in the second interval of each response in the figure. The potential fluctuations across the ten EER's also share a degree of likeness in the latter portion of the first and initial part of the third intervals. However, neither approaches the conformity found in the second interval.

In order to better establish the bounds of the response activity it is necessary to find some quantitative measure of this similarity across the EER's. The degree of sinilarity between two responses for a specific interval can be quantified by a correlation 
coefficient which would provide a total of 45 correlations per interval for the ten EER's of Figure 3.2. This coefficient, $\rho$, can be expressed as:

$$
\rho_{i}=\frac{\Psi x y}{\Psi_{x} \Psi_{y}}
$$

where $\Psi_{x y}$ is the time covariance between EER's $x$ and $y$ in interval $i$ and $\Psi_{x}$ and $\psi_{y}$ are the time standard deviations of these waveforms in this interval. How to reduce these paired correlations to a single measure of the similarity across the entire set of EER's becomes the major concern. The method chosen in this study was to average the correlation coefficient for one interval. This average provides a conservative measure of the conformity across the ten responses since most of the correlation coefficients between waveform pairs must be significant to obtain a significant average correlation. The average correlation reduces the effects of sporadic deviations in these correlation coefficients, which randomly appear between paired waveform segments. The significance of these correlations is questionable, since they do not have any consistent relation to the stimulus.

The correlation coefficients for each paired EER (Figure 3.2) at a specific interval are provided in Tables $B .1$ through $B .7$ in Appendix B. The averages and maxima and minima of these correlations are provided in Table 3.1. The average correlations reinforce the previous observation that the activity in the second interval across the ten EER's is the most consistent. In fact, even the minimum 
TABLE 3.1

Average Correlations for Figure 3.2

$\begin{array}{lrrrrrrr}\text { INTERVAL } & 1 & 2 & 3 & 4 & 5 & 6 & 7 \\ \rho_{\text {ave }} & .30 & .88 & .12 & .05 & .06 & .04 & -.07 \\ \rho_{\max } & .89 & .99 & .88 & .88 & .94 & .87 & .98 \\ \rho_{\min } & -.45 & .58 & -.71 & -.92 & -.87 & -.92 & -.87\end{array}$

correlation of .58 is above the 1 per cent significance level, i.e., assuming samples are normally distributed and independent (Underwood, et al., 1954). The average of the correlation coefficients across the segmented EER's obtained from the wider band potentials were generally less than those in Table 3.1 (see Tables B.8 through B.13). Thus filtering the data appears to eliminate some of the variability across the response estimates.

The first interval of the EER's is the only other 135 millisecond time period where the waveforms demonstrate ary degree of consistent correlation. This consistency appears to be the result of the more common components in the latter half of this interval rather than the initial deflections after stimulus onset. In intervals 3 through 7 (i.e., 270 to 950 milliseconds after the stimulus) the EER's demonstrate negligible correlation. This variability of these late components of the EER seems to indicate that: (1) the average background activity is much greater than the average response activity during this period; (2) the individuality of the response for different subjects is greater, which increases the deviations of the estimate 
in this region; or (3) the variations with stimulus number are greater in the late response. In similar correlation tests, Perry and Childers (1969) have demonstrated a significant decrease in the average correlation coefficients of inter-subject VER's following the first 300 milliseconds of the response, and a decrease in intrasubject correlations after 500 nilliseconds. They also note that the highest correlations are generally found in the second and third 100 millisecond intervals, which is in close agreement with the previous results. Therefore, the EER and VER results seem to reject the reasoning that it is the individuality of the evoked response which introduces the variability in the late components of the ensemble average.

It is interesting to note the occurrence of alpha-like activity in the latter portion of response 40 . This singular occurrence may be the result of a particular combination of the random EEG potentials. However, these potential fluctuations have a strong resemblance to the after-discharge activity noted in the studies mentioned in Chapter 2.

In Figure 3.3 the ten EER waveforms are superimposed with the time mean removed from each average. In this figure the consistency of the responses in the region from about 60 to 300 milliseconds after the stimulus can easily be seen. Because of the similarity of the EER in this region $\left(R_{2}\right)$ it will be designated the primary response region. In this region there are three prominent peaks. These peaks are identified in Figure 3.3 as components one, two 
and three, and occur approximately $90 \mathrm{msec}, 150 \mathrm{msec}$, and $250 \mathrm{msec}$ after stimulus onset, respectively.

The average correlation coefficient in the primary response region is 0.75 , which is slightly less than the average correlation in the second interval of Figure 3.2. This decrease is probably the result of the increase in interval width from 135 to $240 \mathrm{msec}$. Thus, the second region of Figure 3.3 has more components and greater variability than the second interval of Figure 3.2. The average correlation of the third region in Figure 3.3 is 0.23 , which indicates a minor degree of similarity not previously found in the corresponding correlations in Table 3.1. This similarity is possibly the result of some low frequency activity common to the tails of the EER's which would not be detected by the correlations of the 135 millisecond

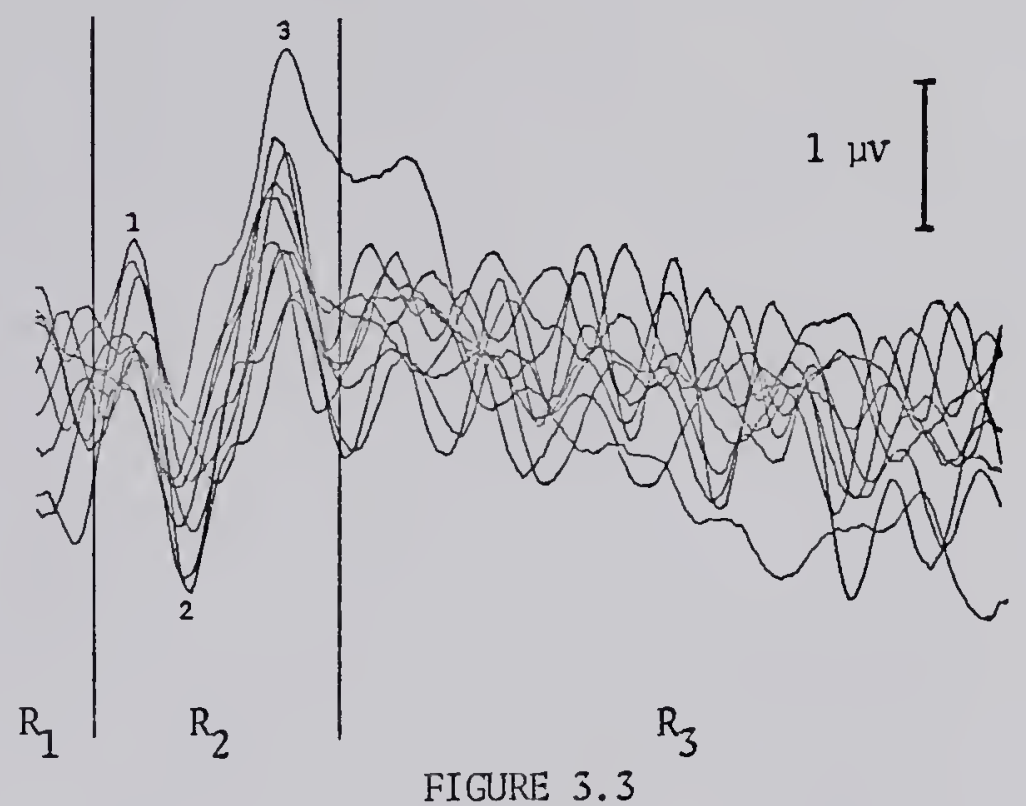

Superimposed Ensembie Evoked Response Overlapping EER's are divided into three regions for comparison. $R_{1}=0$ to $60 \mathrm{msec} ; R_{2}=60$ to $300 \mathrm{msec} ; R_{3}=300$ to $950 \mathrm{msec}$. 
intervals. However such a low frequency conponent is not visually detectable, except for a very slight negative drift in the late response components.

\subsection{Noise in the Ensemble Evoked Response}

The EER's in the previous figures contain an unknown amount of background noise. One estimate of the amplitude of the background activity which remains in the EER is the ensemble average under non-stimulus (control) conditions. The accuracy of this estimate is dependent on the relationship between the signal and the noise. For example, if the signal and noise are independent and the noise is stationary, this average provides a good measure of the amount of background activity in the EER. The waveshape of this average is important only if a particular component appears time locked to the occluded stimulus, indicating the existence of some experimental artifact.

In Figure 3.4, this average is subdivided into ten contrcl waveforms attending the ten occluded stimuli. The format of this figure is identical to that of Figure 3.2. The average correlations of the

TABLE 3.2

Average Correlations for Figure 3.4

$\begin{array}{lccccccc}\text { INTERVAL } & 1 & 2 & 3 & 4 & 5 & 6 & 7 \\ \rho_{\text {ave }} & .07 & -.02 & -.03 & .01 & -.07 & -.00 & -.07 \\ \rho_{\operatorname{lnax}} & .81 & .92 & .95 & .99 & .87 & .92 & .81 \\ \rho_{\min } & -.79 & -.99 & -.93 & -.92 & -.98 & -.93 & -.93\end{array}$


Interval

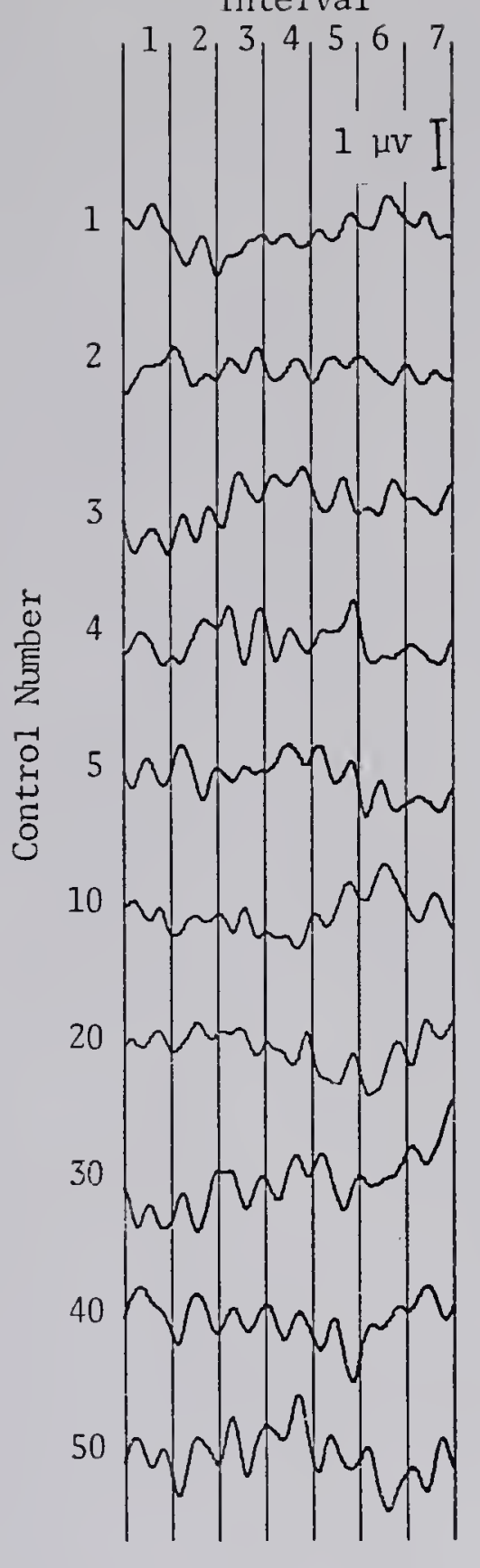

FIGURE 3.4

Ensemble Control Averages

Ensemile averages for ten occluded stimuli are divided into 7 intervals of 135 milliscconds. intervals of Figure 3.4 given in Table 3.2 demonstrate the lack of correlation between the control waveforms. These correlations are similar to the average EER correlations in the last four intervals of Table 3.1. This supports the previous observation that there may be little response activity in this region.

In every interval the root mean square of the control waveforms, $\mathrm{C}_{\mathrm{rms}}$, is less than the rms value of the responses, $R_{\text {rms }}$, in the second interval of Figures 3.2 (where these rms values have the dc removed). The minimu $R_{\text {rms }}$ is only 1.03 times the maximum $\mathrm{C}_{\mathrm{rms}}$ and the magnitude of the largest $R_{\text {rms }}$ is 9.7 times the smallest $\mathrm{C}_{\mathrm{rms}}$. The average of all ratios of $\mathrm{R}_{\mathrm{rms}}$ to $C_{\text {rms }}$ is 2.6. Therefore, assuming that the ensemble control average provides a good estimate of the amount of background activity remaining in the averaged response and that the signal and noise are additive, what is the signal to noise ratio $(S / N)$ in the EER? To date, the answer to this question cannot be resolved, since it is 
necessary to also krow the phase of the average background activity. However, with only a magnitude estimate, the upper and lower bounds of this signal to noise ratio can be found by adding and subtracting the magnitude of the control from the EER and dividing by the control amplitude, i.e.,

$$
\frac{R_{r m s}-C_{r m s}}{C_{r m s}} \leqq S / N \leqq \frac{R_{r m s}+C_{r m s}}{C_{r m s}}
$$

It should be noted that in the case when the lower bound is negative (which is a theoretically impossible event) the lower bound is set to zero.

Rather than establish these bounds, a more common practice is to estimate the signal to noise ratio in the average response by dividing the magnitude of the average response, $\mathrm{R}_{\mathrm{rms}}$, by the average noise, $C_{\text {rms }}$ (Perry, 1966), i.e.,

$$
\frac{\mathrm{S}}{\mathrm{N}}=\frac{\mathrm{R}_{\mathrm{rms}}}{\mathrm{C}_{\mathrm{rms}}}
$$

Using this estimate, the relative signal to noise measures on the previous page provide the maximum, minimum and average $\mathrm{S} / \mathrm{N}$. Thus, one would predict that the EER's in the second interval of Figure 3.2 contain approximate1y 10 to 50 per cent noise with an expected value of 28 per cent. In Chapter 4 it is shown that under certain assumptions these predicted $S / N$ values are reinforced by the degree of similarity (i.e., correlation) previously found between the EER's. Therefore, the average amplitude under control conditions probably provides a reasonabie cstimate of the amount of noise distorting the 
EER wave form.

If the overall rms value of the conirol waveforms (approxinately $0.5 \mu \mathrm{v}$ ) represents the expected noise distortion in the response average, then Figure 3.5 provides an upper and lower bound of the noise distortion expected in the EER. The actual magnitude of the background noise potentials at any instant could be several times their rms value. However, the bounds in Figure 3.5 provide an average approximation of the amplitude of the background noise which could be contained in the EER estimate. That is, the average response without noise should be expected to be within these bounds if the control waveforms provide a good estimate of background noise and if the EER is a good estimate of the average signal plus noise activity. The latter stipulation, the quality of the EER estimate, provides further confidence limits in the average evoked activity. Thus a more accurate bound on the evoked activity is a combination of the noise bounds and the confidence limits on the EER.

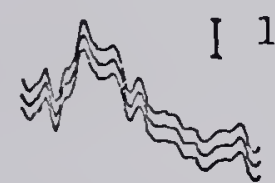

1

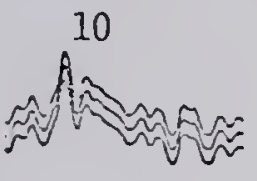

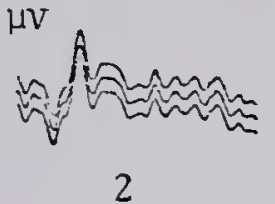

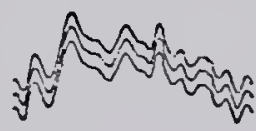

3

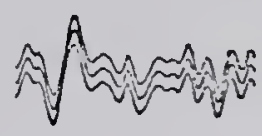

4

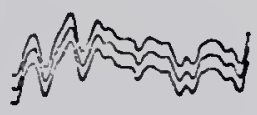

5

Response Number
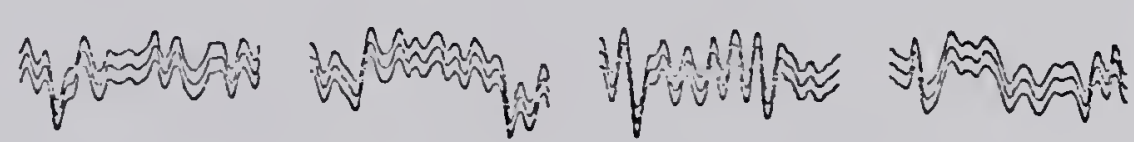

FIGURE 3.5

Noise Amplitude in EER

Overall ms control value is added and subtracted from the center EER waveform. 


\section{5 guality of the Ensemble Evoked Response Estimate}

The EER is a good estimator of the signal plus noise mean if the deviations of this estimate about the mean are relatively minor. A measure of this estimation deviation is provided by the standard deviation, $\sigma_{f}(t)$, of the underlying process. The actual value of $\sigma_{f}(t)$ is unknown. However, an unbiased estimate of $\sigma_{f}(t)$ is the ensemble standard deviation, $\tilde{\sigma}_{f}(t)$, which is calculated from the equation

$$
\tilde{\sigma}_{f}(t)=\left\{\frac{1}{N-1} \sum_{i=1}^{N}\left[f_{i}(t)-\tilde{m}_{f}(t)\right]^{2}\right\}^{1 / 2}
$$

where $N$ is the number of $f_{i}(t)$ 's, subject EEG's, and $m_{f}(t)$ is the ensemble average given by equation 2.2 .

A plot of $\tilde{\sigma}_{f}(t)$ for the potentials attending each of the ten is provided in Figure B.5. These ten waveforms are superimposed in Figure 3.6 in order to observe any stimulus effects on $\tilde{\sigma}_{f}(t)$. As

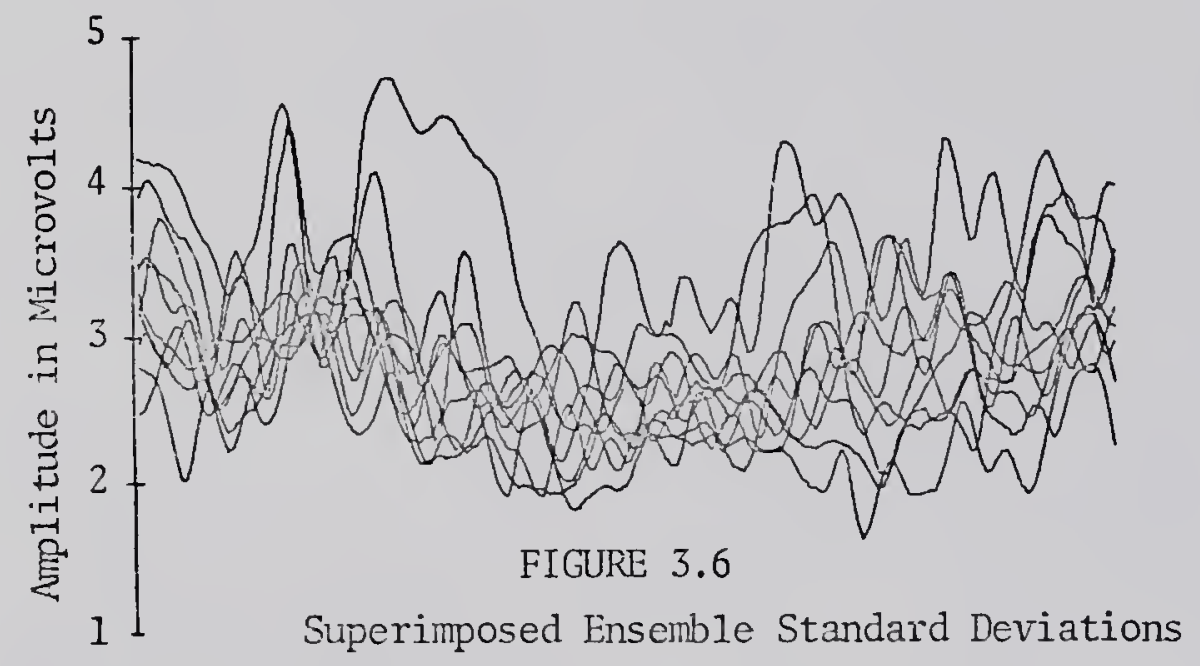

Each standard deviation attends one of the ten selected stimuli which occur at the extreme left of the figure. 
can be seen in this figure, the fluctuations of the $\tilde{\sigma}_{f}(t)$ waveforms do not appear to have any consistent relationship to the stimulus, other than a slight average increase around the primary response region.

If the ensemble standard deviations in Figure 3.6 provide a good estimate of the standard deviations of the process, then the standard deviation of the mean estimate, $\tilde{m}_{f}(t)$, is

$$
\operatorname{sd}\left[\tilde{\mathrm{m}}_{f}(t)\right] \simeq \frac{\tilde{\sigma}_{f}(t)}{\sqrt{N}}
$$

This standard deviation can be incorporated into the Tchebycheff inequality to provide reliability or confidence bounds on the EER estimate. This inequality provides a lower bound on the probability that the true mean and estimated mean will differ by less than $n$ standard deviations of the estimate. For example, we can be at least 89 per cent confident that the true mean and estimated mean (EER) will differ by no more than three standard deviations (calculated by Equation 3.5) of the estimate, regardless of the underlying probability distribution. If the underlying probability distribution is Gaussian, the confidence increases to 99 per cent.

The 89 per cent confidence intervals for ten EER's are plotted in Figure 3.7. The bounds in this figure are constructed from ten EER waveforms plus or minus three-sevenths of the ensemble standard deviations superimposed in Figure 3.6. As can be seen in Figure 3.7, or calculated from Figure 3.6, the overall width of the intervals varies from about 2 to $4 \mu v$. Although this appears to be a relatively 
large amount of variability, the shape of the bounding waveforms generally conforms to the EER waveshape, with a few exceptions. The most notable exception occurs in the first response, around 300 milliseconds after stimulus onset. This variation could have been anticipated from Figure 3.6, since the standard deviation of the potentials in this region of the first response partially stands above the remaining response deviations.

If the confidence intervals in Figure 3.7 are compared with the VER confidence intervals in Figure 3.8, the reliability of the VER is greater than the EER in two out of the three cases shown (J.M. and B.A.). In the third case (J.F.) the reliabilities are approximately equa1, if the greater amplitude of the VER is taken into consideration. In all three cases the VER's were obtained by averaging the subject's response to fifty stimuli. Therefore, for
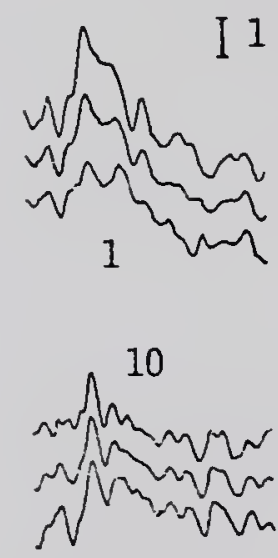

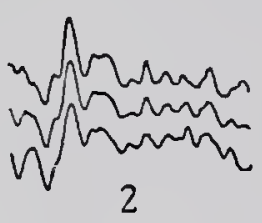

20

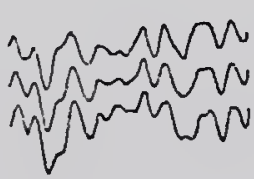

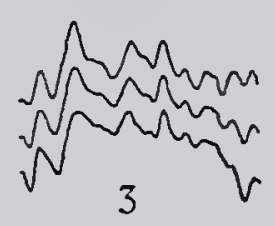

Response Number

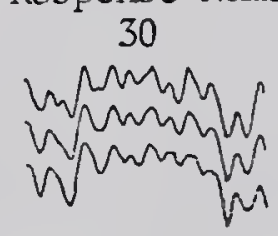

FIGURE 3.7

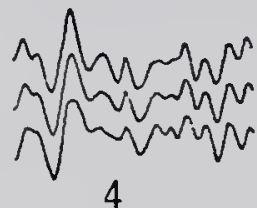

4
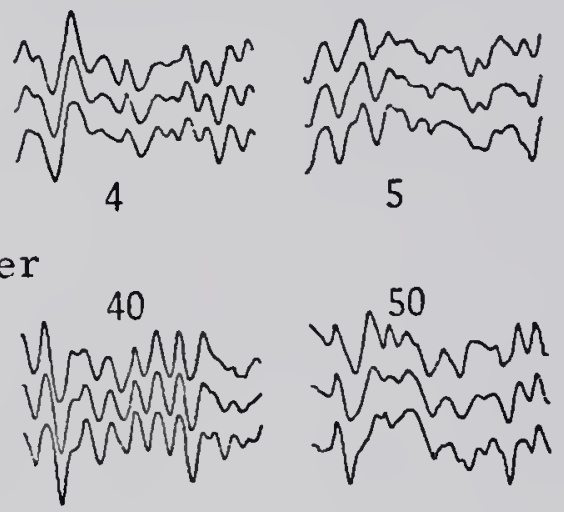

EER Confidence Intervals

The above intervals are plus and minus three standard deviations of the EER estimate. This is about $3 / 7$ of the deviations in Figure 3.6. 
the same number of averages, the EER appears to provide as good an estimate of the response activity as some VER's.

The VER amplitude is about one and one-half times that of the EER's in all of the cases shown. This larger amplitude and greater reliability of the VER estimate implies that the inter-subject response variability is greater than the variability of responses obtained from a single subject. This is what one would predict from the comparison of VER's obtained from a single subject and those obtained from several subjects. In addition, the background noise variability would influence the reliability of the estimate. Thus inter-subject EEG variability (e.g., amplitude and frequency deviation in the background activity) probably reduces the confidence in the EER estimate. One variable parameter which might reduce confidence in the VER and not in the EER would be a general change in the evoked activity with the number of stimuli. In order for this variation not to affect the EER it is necessary for this change to be fairly consistent across the stimuli for the subject population.

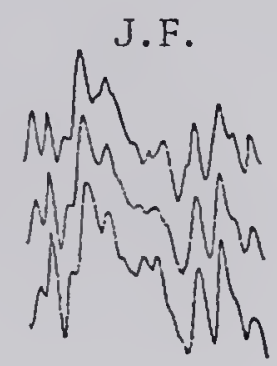

J.M. B.A.
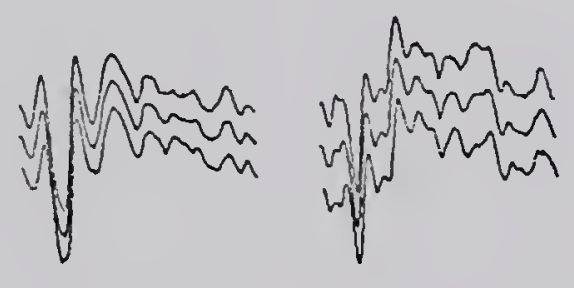

I $1 \mu v$

FIGURE 3.8

VER Confidence Intervals

Scale and procedure is the same as in Figure 3.7. Each of the above subjects are members of the ensemble. 
3.6 Variations in the Ensemble Evoked Response with Stimulus Number As noted previously, the EER provides an estinate of the changes in the evoked response (common to the subject population) with repetitions of the stimulus. That is, each EER in Figure 3.2 is an estimate of the activity which is time locked to a specific stimulus within the periodic train. In order to obtain an accurate estimate of the response changes related to the stimulus number, it is necessary to remove the EER variations resulting from the background noise. Since this cannot be accomplished at this time, the EER's across stimuli will be examined for trends of change, noting that variations caused by the background noise should be random in nature.

In a preliminary investigation of the EER's in Figure 3.2 as a function of stimulus number, little transient change is discemible. The only noticeable change is a general decrease in amplituce between the first and remaining responses. The first EER appears to contain a low frequency component, particularly evident in the latter portion of this response, which is not as predominant in the EER's that follow. In the superimposed EER's in Figure 3.3, this low frequency activity causes the initial components of the first response to rise above the other responses and the later components to descend below the remaining responses. In addition, the EER to the first stimulus contains a sustained positive component about $300 \mathrm{msec}$, which does not have a counterpart in any of the following EER's. However, the reliability of this component is questionable because of the increase in the ensemble standard deviation in this region. It therefore 
appears that not only does the average response to the first stimuli differ from those that follow, but that the predominant component (around the third peak of Figure 3.3) of this response is more variant than any other.

If the activity outside of the primary response region (60 to $300 \mathrm{msec}$ ) in Figure 3.3 is ignored and the means within the correlated section are removed from each response, the amplitude of the first response in this region does not stand out. For example, if the magnitude of the waveforms in the primazy response region are plotted as a function of stimulus number, the amplitude of the first response is no longer so predominant. A plot of both the rms and peak to peak, ptp, amplitude variations for the individual response in the interval is provided in Figure 3.9. For this figure the rms value is about a zero mean and the ptp value was measured from the second and third primary deflections indicated in Figure 3.3.

Since the responses are very similar in this region, the rms and ptp measures provide essentially the same infornation. Both demonstrate a slight decreasing trend in amplitude with the number of stimulus presentations. However, the magnitude of the amplitude fluctuations is not much smaller than the overall decrease; therefore the significance of the latter appears questionable. In a plot (Figure 3.10 ) of the changes in the time delay or latency of the three peaks in the 60 to $600 \mathrm{millisecond}$ interval no significant trend is discernible. Therefore, the above results imply that little, if any, transient change exists in the first fifty responses for 


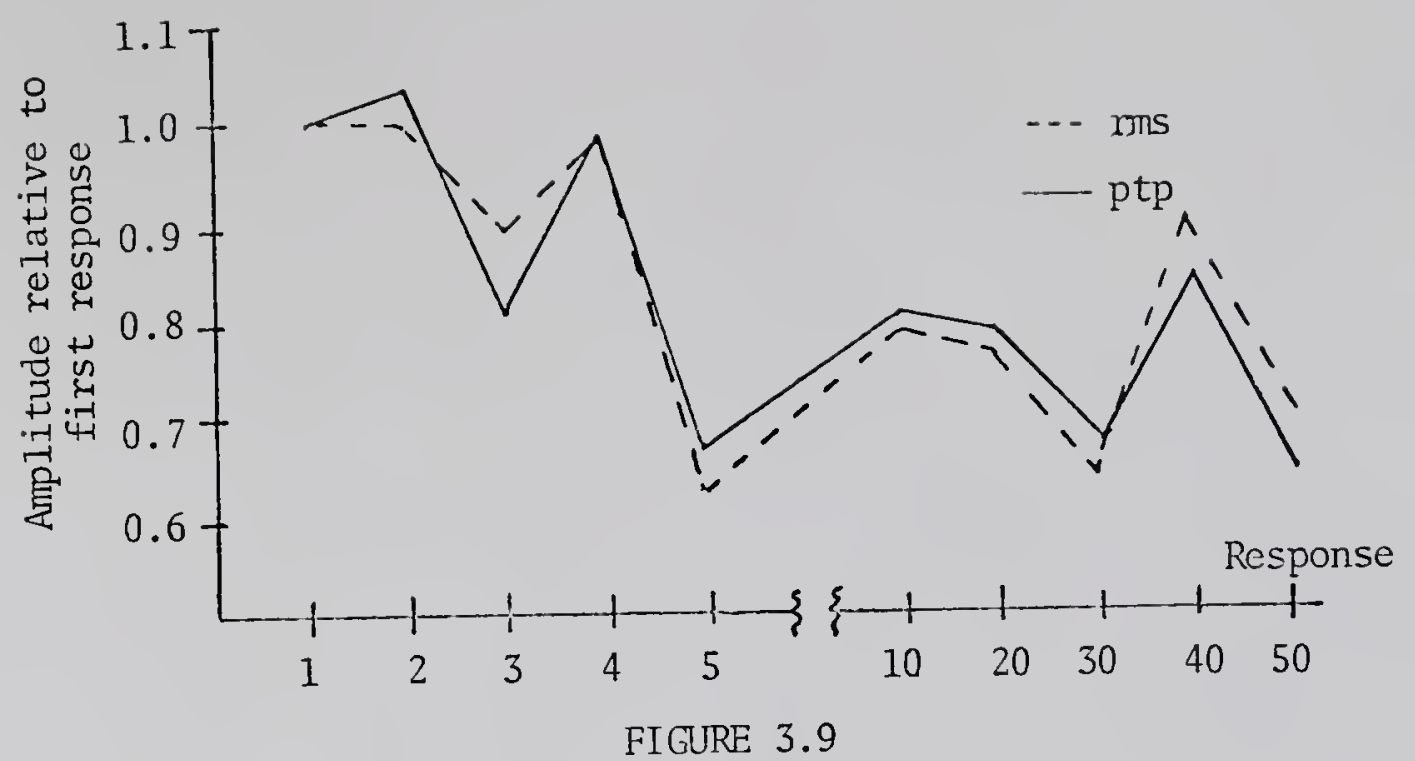

Amplitude Fluctuations of the Primary Response

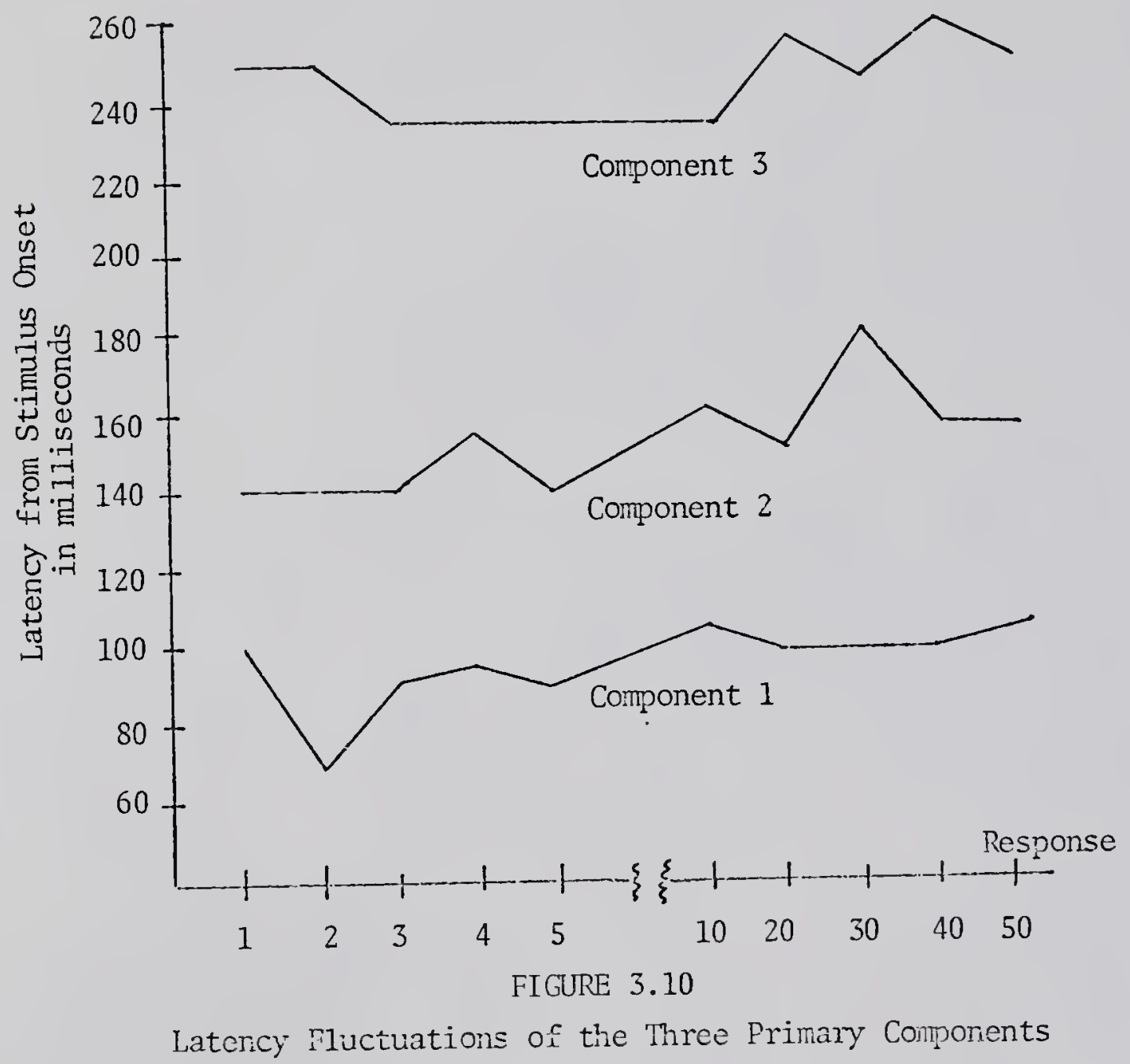


responses averaged across a subject population. An exception to this observation is the difference in the overall character between the first and remaining responses. This may be evidence of some initialization of the visual system either at the receptor or higher neural processing level. At the receptor leve1, Brazier (1967) has noted that there is a large difference in the amplitudes of the electroretinogram attending the first and second stimuli in a repetitive train. However, she also notes that the armplitude of the second ERG is smaller than any that follow, indicating that the transient change at the receptor input exists beyond the first flash. The persistent transient variations (11 to 20 seconds) noted by Regan (1966b) in responses recorded at the scalp also imply a more sustained initialization period than is found in the above EER results. However, Regan utilized a continuous sinusoidal stimulus which restricts extrapolation of his results to the present study. If initialization is not a factor then a high degree of variability in the first response might account for the relative uniqueness of the EER to the first flash. The ensemble standard deviation following the first stimulus partially supports this reasoning but it does not explain all of the component differences between the first and succeeding EER's. Therefore, system initialization and inter-subject response deviation may both contribute to this difference.

\subsection{Average of Ensemble Evoked Responses}

The results of the previous sections indicate that the EER provides a relatively stable response estimate in the region from 60 to 300 

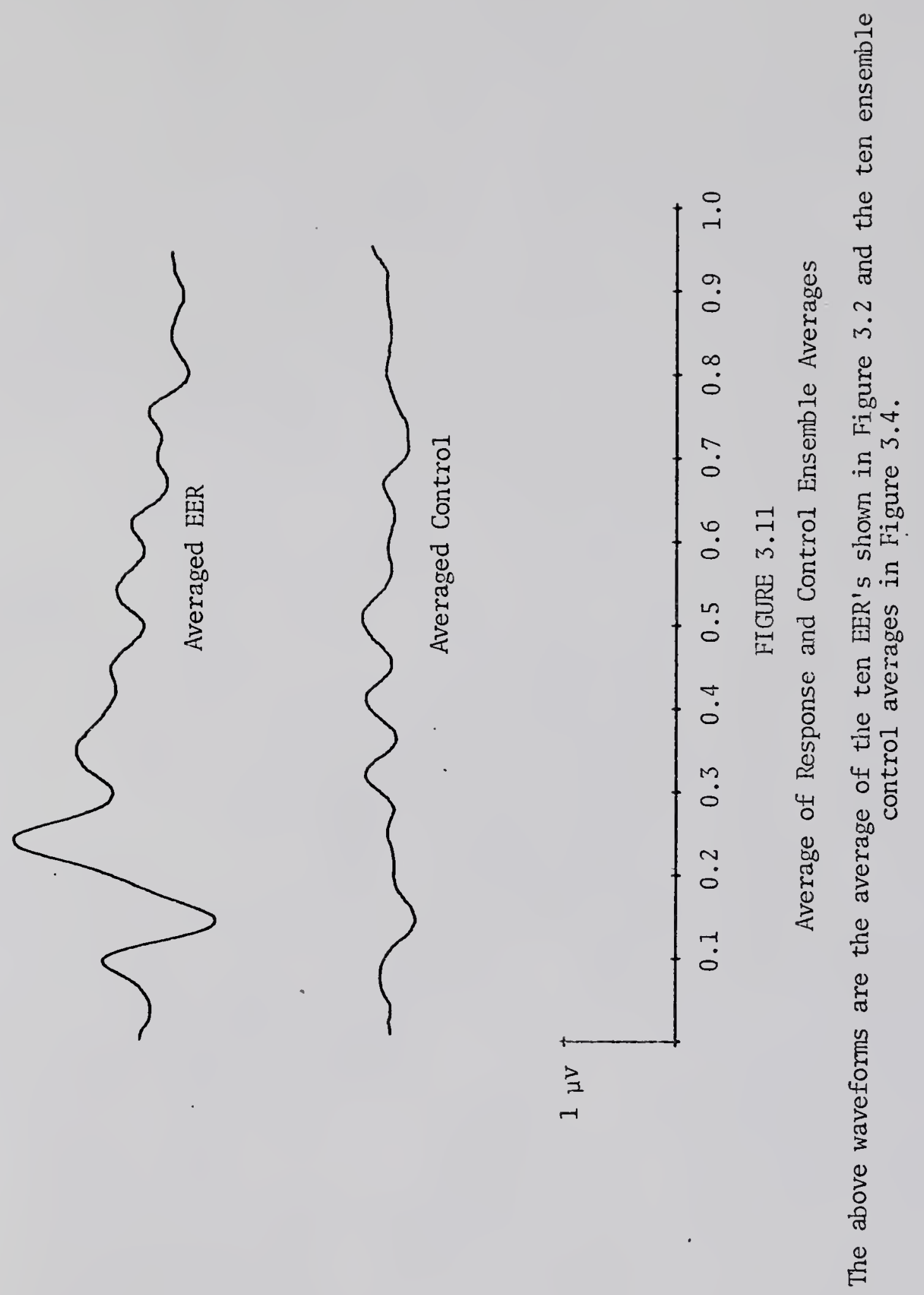
msec. If this region is stipulated to contain the primary activity of the evoked response, then very little response information should be lost by averaging EER estimates across stimuli. This average should further reduce the average background noise activity and increase the quality of the response estimate. That is, the variance of the background noise and the variance of the EER estimate should both decrease with the increase in the total number of averages from fifty to five hundred.

Figure 3.11 provides the average of the ten EER's and the ten control waveforms shown previously. As can be seen in this figure, this average seems to extract the near sinusoidal complex in the primary region and reduce the remaining components. The averaged control is a general overall reduction of the individual control waveforms presented earlier. The $\mathrm{S} / \mathrm{N}$ ratio in the primary response region of the averaged EER is estimated from the averaged control to be about 5.6. This is a two-fold improvement over the average $\mathrm{S} / \mathrm{N}$ ratio estimated for the EER to a single response from the individual control waveforms. If confidence intervals were provided for the averaged EER, each interval would be less than $0.5 \mu \mathrm{v}$ from this average. Therefore the average of the ten EER's provides a reliable, relatively noise-free inter-subject response norm.

\subsection{Sumnary}

The average over an ensemble of subject EEG's (EER) extracts a relatively consistent estimate of the response to visual stimuli. The consistency of ten EER's is found to be greatest for components 
in the 60 to $300 \mathrm{msec}$ region following stimulus onset. The variations of these components with stimulus number are generally insignificant, indicating little change, either habitual or othenvise, in the activity evoked by the first fifty stimuli. However, a general modification in the character of the entire average waveform is noted following the EER to the first stimulus. It is suggested that this change is the result of initialization of the visual system and a greater variability of the first response across the subject population. This variability of the first response is demonstrated in confidence intervals about the ten EER estimates.

The amount of noise activity in the more consistent region of the EER's is estimated by ensemble control averages. From these estimates the average $S / N$ ratio in the EER is found to be 2.6 . In order to further extract the response signal from the noise, the average of the ten EER's is calculated. This averaged EER provides a more reliable response estimate than single EER's and improves the $S / N$ ratio. The response estimate extracted by this average is almost an inverted sinusoid, beginning approximately $100 \mathrm{msec}$ after stimulus onset. 
CHAPTER 4

THEORETICAL ASSUMPTIONS UNDERLYING RESPONSE AVERAGING

In order to theoreticaily justify the results obtained from many EEG data processing techniques, it is necessary to assume certain hypotheses about the statistical character of the fluctuating potentials. Investigators who employ the averaging techniques to extract time locked signals from the incoherent background noise generally assume that: (1) the noise is a wide sense stationary random process; (2) the signal and noise are independent; and (3) the signal and noise are additive. The latter assumption is probably the most important since non-additive components cannot be separated by the linear process of averaging. However, a signal dependent on nonstationary noise can be extracted if the degree of dependency and non-stationarity is minor.

In this chapter an attempt will be made to gain positive or negative evidence about these assumptions by examining the data obtained from the subject ensemble. The results of these examinations will not, however, enable the experimentor to negate or to confirm these assumptions because of the lack of knowledge about the potentials being tested. Thus, to examine one of the above assumptions, the test utilized must hypothesize the nature of the signal and noise parameters. In many cases the test hypotheses depend on one or two of the above assurmptions, conditioning the conclusiveness of 
the result. The present study is therefore intended to provide insight into the possible workability, rather than ultimate veracity of these assumptions. Such an examination may then provide a foundation for further studies into the statistical properties of evoked signals in background noise.

\subsection{Stationarity of the Background Noise}

A random process is said to be stationary if the statistics of the process are time invariant (see Davenport and Root, 1958). For example, a large number of statistically independent sample functions which are simultaneously recorded constitute a wide sense stationary process if the mean and autocorrelation function at one instant of time equals those at all other times. In the present study the sample functions are the EEG records of the fifty subjects. The time varying function of particular interest is the ongoing activity under stimulus conditions. However, these potentials cannot be separated from the evoked response. Therefore, this investigation must rely on the resting potentials under non-stimulus conditions to estimate the background activity. It then becomes necessary to work under the constraint of the second assumption (i.e., that the signal and noise are independent) to examine the first.

Since an average is an estimate of the first moment of the ensemble, it is the stationarity of background noise mean which is the most critical to the averaging technique. If the noise mean is highly non-stationary (e.g., the averaged potential fluctuations exceed those of the response average) then the averaging process 
cannot extract a detectable signal from the background noise. Utilizing the resting EEG potentials to approximate the background activity, the noise mean estimate (ensemble control average), seen in Figure 3.4, is not time invariant. However, are these fluctuations the result of an underlying non-stationary process or caused by the variations inherent in the estimation process? In order to answer this question it is necessary to determine the inherent variance of the mean estimate of $\mathrm{N}$ sample functions. If the $\mathrm{N}$ sarmple functions are independent, which is plausible since individual subject EEG's should not depend on one another, then this variance of the estimate is (see Bendat and Piersol, 1966)

$$
\operatorname{VAR}\left[\tilde{m}_{f}(t)\right]=\frac{\sigma_{f}^{2}(t)}{N}
$$

where $\sigma_{f}^{2}(t)$ is the variance of the process which is unknown and must be estimated. This latter estimate also has variance associated with it which again must be estimated, and so forth. In order to prevent endless estimation of these statistics, the estimate of the variance, $\tilde{\sigma}_{f}^{2}(t)$, will be assumed equal to the actual variance, $\sigma_{f}^{2}(t), i . e .$,

$$
\tilde{\sigma}_{f}^{2}(t)=\sigma_{f}^{2}(t)
$$

Now utilizing the Tchebycheff inequality, the range of the mean estimate can be predicted without knowing its exact probability distribution. This inequality states that the probability that the difference between the true mean and estimate mean will be greater 
than $\mathrm{C}$ standard deviations of the estimate will be less than $1 / \mathrm{C}^{2}$, i.e.,

$$
\operatorname{PROB}\left[\left|\tilde{m}_{f}(t)-m_{f}(t)\right| \geq C\left\{s d\left[\tilde{m}_{f}(t)\right]\right\}\right] \geq \frac{1}{C^{2}}
$$

In Chapter 3 this inequality was used to provide 89 per cent confidence linits $(\mathrm{C}=3)$ about the EER and VER estimate, noting that

$$
\operatorname{sd}\left[\tilde{m}_{f}(t)\right]=\frac{\tilde{\sigma}_{f}(t)}{\sqrt{N}}
$$

Similar confidence limits can be obtained by hypothesizing a mean and using $\tilde{\sigma}_{f}(t)$ to obtain confidence limits. For example, if the noise mean, $m_{f}(t)$, is hypothesized to be stationary and equal to zero, then Equation 4.3 becomes

$$
\operatorname{PROB}\left[\left|\tilde{m}_{f}(t)\right| \geq C\left\{\tilde{\sigma}_{f}(t) / \sqrt{N}\right\}\right] \leq \frac{1}{C^{2}}
$$

Again setting $C=3$, the 89 per cent confidence intervals about the hypothesized stationary mean can be established. In addition, from Equation 4.5 another value of $C$ can be calculated from the ensemble value of $\tilde{m}_{f}(t)$, the ensemble control average, and $\tilde{\sigma}_{f}(t)$, the control standard deviation, i.e.,

$$
C \leq \sqrt{\bar{N}} \frac{\tilde{m}_{f}(t)}{\tilde{\sigma}_{f}(t)}
$$

The maximum value of $\mathrm{C}$ found in Equation 4.6 can then be compared to the threshold value of 3 -- rejecting the stationary hypothesis for $C>3$ and accepting the hypothesis for $C<3$. This is equivalent 
subject, i.e., near deteministic, then the resultant average should contain mainly background activity. The reliability of this estimate is, however, contingent upon the third assumption (i.e., that the signal and noise are additive) in addition to the second.

With this restriction in mind, the preceding test for stationarity can also be applied to the plus-minus average. The standard deviation of this estimate about the true \pm average is the same as the standard deviation of $m_{f}(t)$ provided in Equation 4.4 since $+\sigma_{f}^{2}(t)$ is the same as the variance, $\sigma_{f}^{2}(t)$, of the process (see Schimmel) under stimulus conditions. By assuming the response signal to be near deterministic, this variance is mainly the result of the background activity. If we again assume that the estimate of the variance is very near the actual variance, the values for $C_{\max }$ can be calculated. The values for $C_{\max }$ provided in Table 4.2 again indicate that the first moment of the background activity is stationary, i.e., the stationary hypothesis is accepted for every \pm average.

If the signal is assumed to be non-deterministic, it contributes to the estimated variance of the process and the below $\mathrm{C}_{\max }$ values

TABLE 4.2

Maximum Number of Standard Deviations of \pm Estimate about Zero Mean

$\begin{array}{lllllllllll}\text { Response } & 1 & 2 & 3 & 4 & 5 & 10 & 20 & 30 & 40 & 50\end{array}$

$\begin{array}{lllllllllll}C_{\max } & 1.93 & 2.34 & 2.69 & 2.00 & 2.21 & 2.14 & 2.30 & 2.49 & 2.27 & 2.82\end{array}$


increase. That is, the noise variance is now the estimated ensemble variance, $\tilde{\sigma}_{f}^{2}(t)$, minus the signal variance. In addition, the \pm average will contain some signal activity. In order to accept the stationary mean hypothesis for the ten \pm estimates the standard deviation of the signal cannot be greater than 10 per cent of the total, $\tilde{\sigma}_{f}(t)$. Such a limitation is reasonable since present magnitude estimates of the evoked response indicate that the background EEG activity has an amplitude about five to ten times as great as the response (Kitasato and Hatsuda, 1965; Perry and Childers, 1969). Therefore, with the incoherent nature of the noise and time locked character of the signal, the ensemble variability of the former should be at least ten times greater than the variations of the latter.

In order to check the validity of the previous test, it is interesting to examine the stationarity of the response average, i.e., EER. Again, assuming that the signal and noise are additive and independent, and that the signal standard deviation is at most 10 per cent of the total ensemble variance, then the values of $C_{\max }$ for the ten EER's in Figure 3.2 are given in Table 4.3.

TABLE 4.3

Maximum Number of Standard Deviations (times 0.1 ) of EER Estimate About Zero Mean

$\begin{array}{lllllllllll}\text { Response } & 1 & 2 & 3 & 4 & 5 & 10 & 20 & 30 & 40 & 50\end{array}$ $\begin{array}{lllllllllll}C_{\max } & 42.5 & 36.1 & 28.1 & 30.1 & 22.6 & 38.3 & 33.0 & 35.8 & 28.6 & 19.0\end{array}$ 
These results clearly demonstrate that the mean of the signal with noise appears to be non-stationary, which is imperative for the signal to be extracted by averaging. Even if the signal variance accounts for the entire ensemble variance, the $C_{\max }$ values calculated (i.e., 1/10 of the above results) would indicate the EER to be non-stationary six out of ten times. Therefore, the preceding stationary mean test can detect non-stationary trends under the worst case conditions, which indicates that it is a valid test.

In order for the background activity to be wide sense stationary the autocorrelation function must also be time invariant. The autocorrelation function, $R(t, t-\tau)$, is a function of both time, $t$, and the time shift of the correlation, $\tau$. For $R(t, t-\tau)$ to be time invariant the autocorrelation function at time $t_{1}$ must be the same at all other time $t_{2}$ for a given $\tau$, i.e.,

$$
R_{f}\left(t_{1}, t_{1}-\tau\right)=R_{f}\left(t_{2}, t_{2}-\tau\right)
$$

The estimate of this function is obtained utilizing Equation 4.9.

$$
\tilde{R}_{f}(t, t-\tau)=\frac{1}{N} \sum_{i=1}^{N} f_{i}(t) f_{i}(t-\tau)
$$

Using Equation 4.9, a three-dinensional plot of the ensemble autocorrelation function estimate versus $t$ and $t-\tau$ for the resting EEG ensemble (Control 1) is provided in Figure 4.1. Since the previous test indicated that the mean is stationary, this average is subtracted from the autocorrelation to eliminate any sampling 


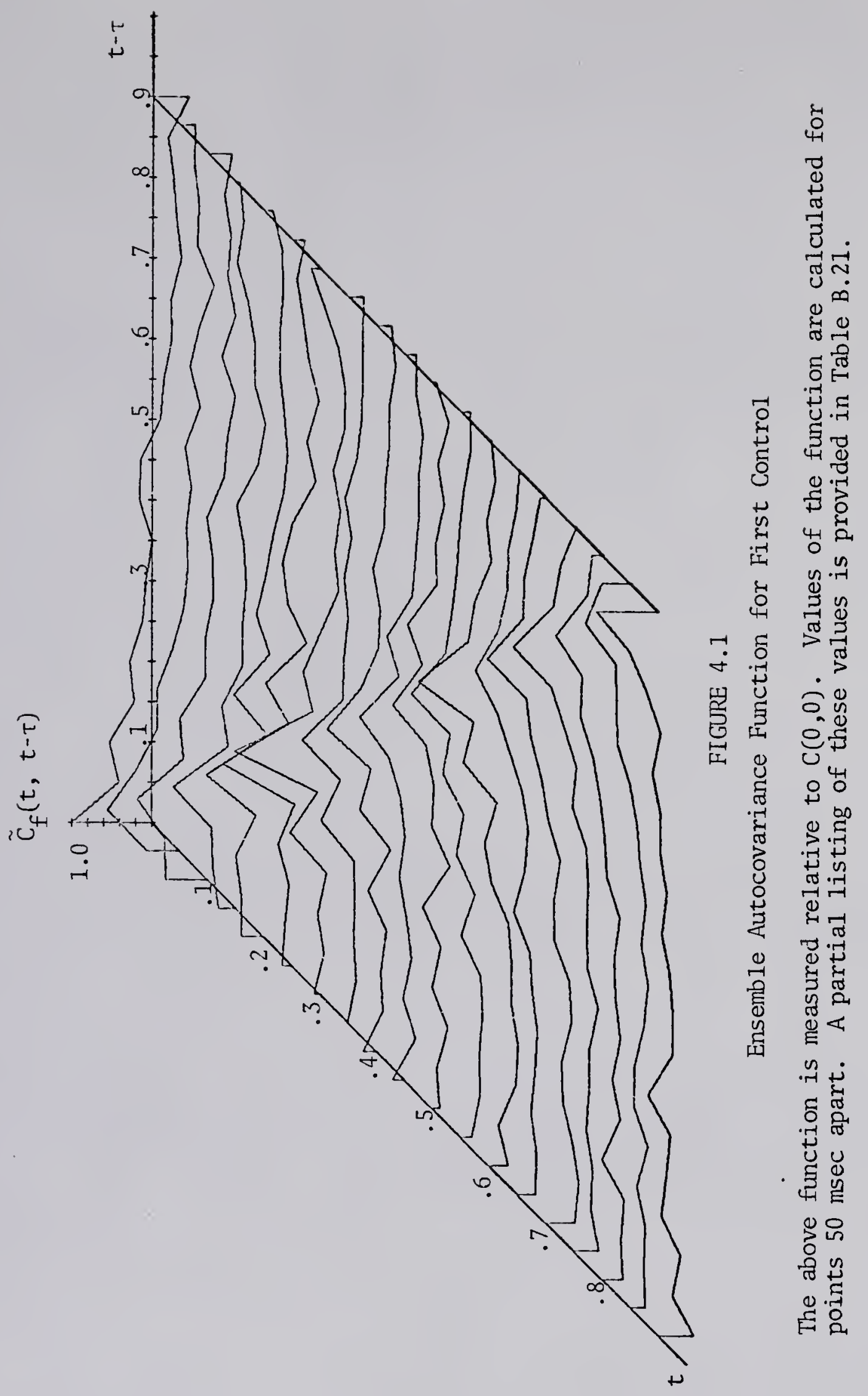


fluctuations of this first moment. Therefore the autocorrelation function in Figure 4.1 is more correctly an autocovariance function which has the form

$$
\tilde{C}_{f}(t, t-\tau)=\frac{1}{N} \sum_{i}^{N}\left[f_{i}(t)-\tilde{m}_{i}(t)\right]\left[f_{i}(t-\tau)-\tilde{m}_{i}(t-\tau)\right]
$$

The magnitude of this function, like the autocorrelation function, is a maximum for $\tau=0$, which occurs along the main diagonal of Figure 4.1. It also should be noted that the covariance function is symmetrical about $\tau=0$; therefore the super and subdiagonal values are equivalent. In order to examine the stationarity of this function one must examine the fluctuations of $\tilde{C}_{f}(t, t-\tau)$ for a fixed $\tau$ and varying $t$, which is analogous to viewing the variation $C$ along the main and off diagonal terms in Figure 4.1. Fluctuations of $\tilde{C}_{f}(t, t-\tau)$ with time are noticeable in this figure; however, their significance is questionable. From the actual values of $\tilde{C}_{f}(t, t-\tau)$ provided in Table B.21 of Appendix B, one does notice that the magnitude of these fluctuations is generally independent of the diagonal used, i.e., the time shift, $\tau$. Therefore fluctuations of the main diagonal for $\tau=0$, which is equal to the ensemble variance, should provide as good a measure as any other $\tilde{C}_{f}\left(t, t-\tau_{1}\right)$ of the time fluctuation of the correlation function.

The ensemble standard deviations of the ten control trials are superimposed in Figure 4.2. These deviations are overlapped to provide a simultaneous estimate of the degree of fluctuation and to provide a comparison with the standard deviations presented in the 
same manner in Figure 3.6. The reader interested in the individual ensemble standard deviations of each control is referred to Figure B.5. The maximum, minimum, and average values of the waveforms in Figure 4.2 are $4.5 \mu v, 1.8 \mu v$, and $2.8 \mu v$, respectively. If the standard deviation is hypothesized to be stationary and equal to the average of these waveforms, then the fluctuations of the estimated values exceed a range of 61 to 160 per cent of this average. This variation is probably greater than one would expect from the estimation error; however, without knowledge of the higher moments one cannot further quantify this statement.

These higher moments are essentially descriptors of the finer detail of the underlying probability structure of the process. Therefore the profile variations of the ensemble probability density function, pdf, should provide a rough evaluation of the degree of

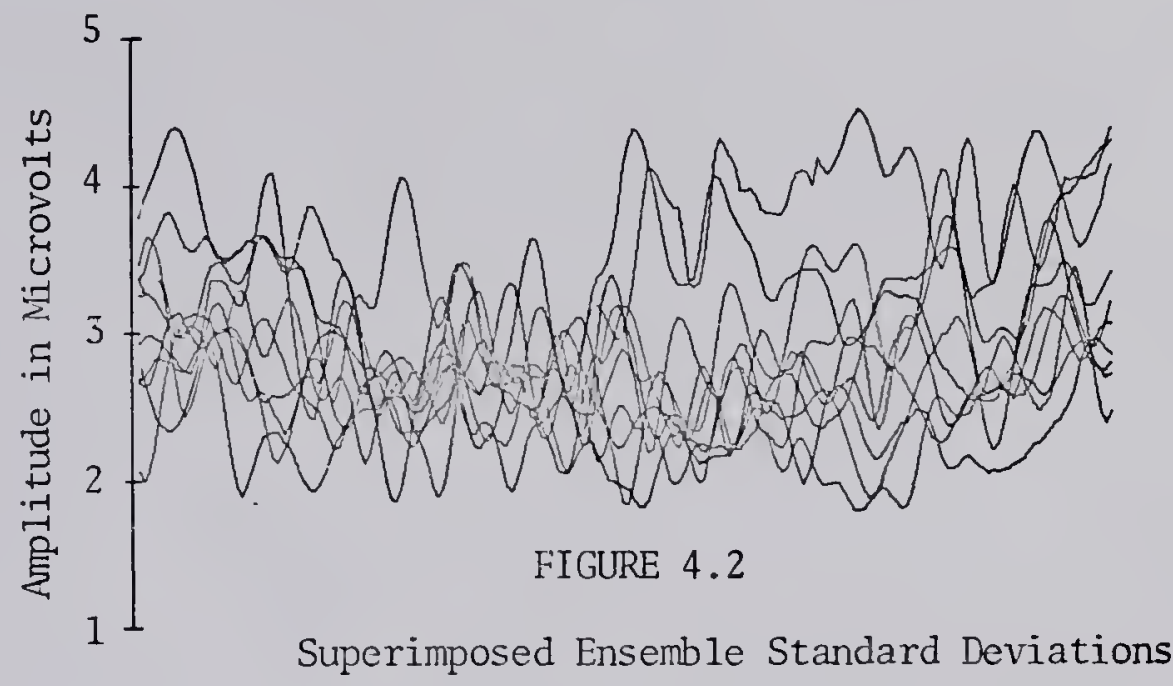

Each standard deviation attends one of the ten occluded stimuli which occur at the extreme left of the figure. 
time dependence of these moments. Previous investigations of the amplitude probability density function of individual EEG records have provided conflicting results. Elul (1968) and Saunders (1963) note that EEG amplitude histograms appear to approximate the normal (Gaussian) distribution. However, the results of a study by Campbe11, et al. (1967) generally reject the hypothesis that the EEG amplitude is normally distributed. A comparison of the ensemble histograms to the nornal distribution may provide additional information about the underlying EEG pdf.

Histogram estimates of the ensemble pdf at every 10 millisecond interval after the first occluded stimulus are shown in Figure 4.3. Each of the seven rows in this figure approximately correspond to the seven 135 millisecond intervals used previously to segment the ensemble control averages (Figure 3.4). As can be seen in Figure 4.3 , there appears to be little consistency between the ensemble histograms at different time instances. The significance of these pdf deviations with time is conditioned by the variance of the estimation process (i.e., the histogram approximation). However, the wide contrast in contour between individual pdf estimates appears to exceed the limitation of sample variability. For example, the histograns in Figure 4.3 vary from umimodal ( $t=60 \mathrm{msec})$ to bimodal $(t=90 \mathrm{msec})$, asymmetric $(t=710 \mathrm{msec})$ to near symnetric $(t=100 \mathrm{msec})$, and leptokurtic $(t=490 \mathrm{msec})$ to platykurtic $(t=330 \mathrm{msec})$.

In addition to this variability, few of the histograms resemble the normal distribution. An attempt to quantify the degree of 


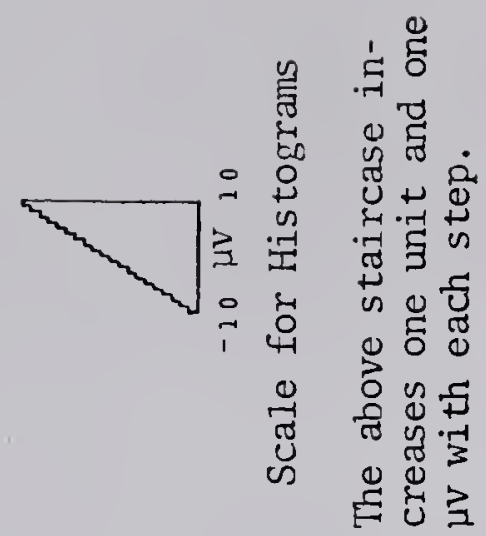

cente

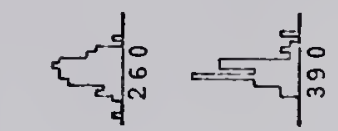

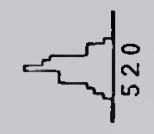

$-\mathrm{H}_{3}$

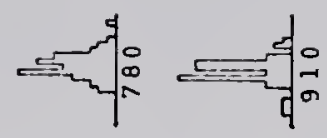

$=$ 국

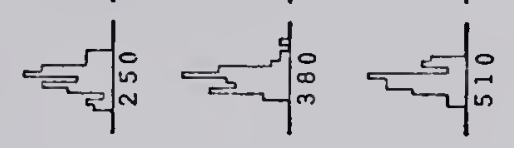

$=-j_{0}$

हो

다:

势

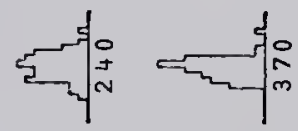

$=-10=-5$

धन क्षं:

है।

to

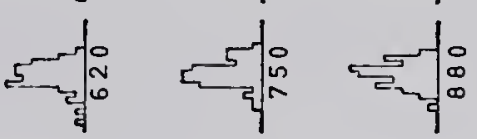

5 .

द

ज्ञ

$=$

$=$

계: 댕ㅎㅇ

$\stackrel{m}{*}$

ज्ञी धन चु०

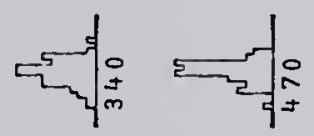

हो:

$5^{5}$

sto

ज्ञात

ris:

हैं: है:

ज्ञां

ज作京

$=-$ सें $^{2}=-$

5ी $=$ को:

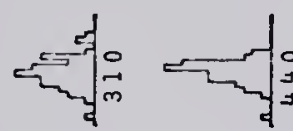

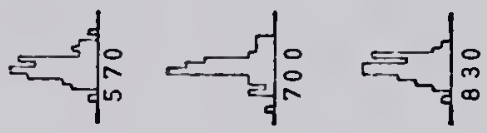

F 웡

जि: =

fio

$=-5$

ri:

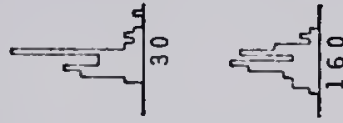

चांक्यें

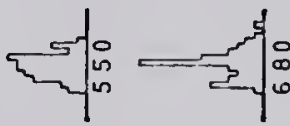

जी

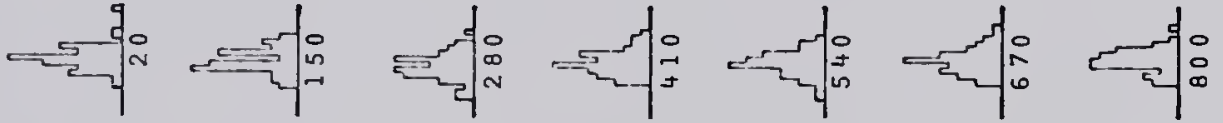

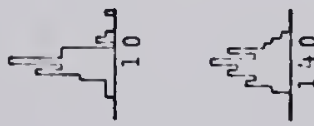

ry

c⿻一㇉:

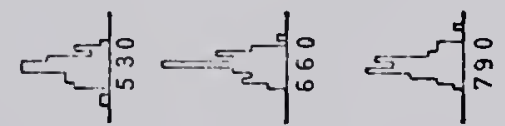


normality utilizing the Kolmogorov-Sinirnov test proved unsuccessful. Although the Kolmogorov-Smirnov test is generally more efficient than the chi-square goodness of fit test (Miller and Freund, 1965) for the number of available samples (50), the necessity of estimating the mean and variance reduced the sensitivity of the test (see Lilliefors, 1967). That is, there was a very high probability of accepting the hypothesis of normality when it was false.

\subsection{Dependence of Signal and Noise}

In the previous section we noted that if the signal and noise are additive and the signal near deterministic, then the plus-minus average should provide an average estimate of background activity. If the same averaging process is performed on the resting EEG, then a similar estimate of the noise under non-stimulus conditions is obtained. A comparison of the general character of these processed potentials should provide an estimate of variations in the average noise under stimulus and non-stimulus conditions. However, how does one make such a comparison? The waveshape of these poientials for ten signal and ten control averages (see Figures B.3 and B.4) has little meaning, since they were recorded at different times. But the degree of fluctuation in the rms measure of the \pm average should provide an estimate of average energy under both conditions. Table 4.4 provides the rms values of each plus minus control and response waveform. The range of rms values is nearly equal under both conditions. Thus, the time fluctuating averages 
TABLE 4.4

RMS Values in $\mu \mathrm{v}$ of \pm Averages

Under Stimulus and Non-Stimulus Conditions

$\begin{array}{lllllllllll}\text { Waveform } & 1 & 2 & 3 & 4 & 5 & 10 & 20 & 30 & 40 & 50\end{array}$

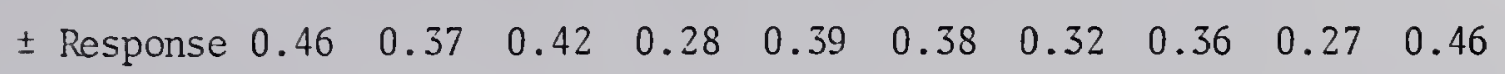

$\begin{array}{lllllllllll} \pm \text { Control } & 0.26 & 0.36 & 0.40 & 0.38 & 0.32 & 0.28 & 0.49 & 0.58 & 0.45 & 0.67\end{array}$

of the background activity and resting activity are of the same magnitude. Since the signal potentials are present in one case and not the other, the general implication is that the magnitude of the average noise is independent of the signal.

Another property of the background and resting EEG which can be investigated under the assumptions of a near deterministic signal added to noise $(s+n)$, is the ensemble standard deviation. If the signal and noise are independent, the standard deviation of the resting EEG ensemble (Figure 4.2) should have a character similar to the standard deviation of the stimulus provoked activity (Figure 3.6). That is, the ensemble standard deviation of signal plus noise, $\sigma_{s \div n}(t)$, is given by

$$
\sigma_{s+n}(t)=\left[\sigma_{s}^{2}(t)+\sigma_{n}^{2}(t)+2 \sigma_{s n}^{2}(t)\right]^{1 / 2}
$$

If the signal is near deterninistic and independent from the noise, then the signal variance, $\sigma_{S}^{2}(t)$, should be much less than the noise variance, $\sigma_{n}^{2}(t)$, and the covariance between the signal and noise, $\sigma_{\mathrm{sn}}^{2}(t)$, equals zero, which reduces Equation 4.11 to the following: 


$$
\sigma_{s+n}(t) \simeq \sigma_{n}(t)
$$

Thus, the standard deviation of the signal plus noise ensemble is primarily the result of the background noise and should be similar to the standard deviation of the resting EEG, if the stimulus does not affect the noise (i.e., signal and noise are independent). As noted previously, the time fluctuations of the standard deviations should not be the same for both cases, since these deviations were recorded at different times. If we compare the standard deviations, $\tilde{\sigma}_{s+n}(t)$ and $\tilde{\sigma}_{n}(t)$, provided in Figures 3.6 and 4.2 respectively, the overall average and peak to peak values are very similar, i.e.,

$$
\begin{array}{ll}
\text { ave }\left[\tilde{\sigma}_{s+n}(t)\right]=2.82 \mu \mathrm{v} & \text { ave }\left[\tilde{\sigma}_{n}(t)\right]=2.86 \mu \mathrm{v} \\
\operatorname{ptp}\left[\tilde{\sigma}_{s+n}(t)\right]=3.1 \mu \mathrm{v} & \operatorname{ptp}\left[\tilde{\sigma}_{n}(t)\right]=2.7 \mu \mathrm{v}
\end{array}
$$

From these measurements one would predict that the variance of the noise is independent of the signal. However, in Figure 3.6 a slight increase in the average standard deviation, $\tilde{\sigma}_{s+n}(t)$, was noted around the primary response region. Since similar trends do not appear in the standard deviations, $\tilde{\sigma}_{n}(t)$, in Figure 4.2 , this increase could imply some signal and noise dependence.

If the near deterministic signal and noise are dependent and the average value of the noise is small, then the ensemble standard deviation of signal plus noise becomes

$$
\sigma_{s+n}(t)=\left\{\sigma_{n}^{2}(t)+E[s(t) n(t)]\right\}
$$

where $E[s(t) n(t)]$ is the mathematical expectation or stochastic average of the product $s(t) n(t)$. 
The ensemble standard deviation for noise dependent on a deterministic signal should then increase when the amplitude of the signal increases. This is the case in Figure 3.6, particularly for the standard deviation of the first response (see Figure 3.1). However, if the signal is relatively non-deterministic, then the standard deviation of independent signal and noise would also tend to increase in the region of greatest signal variability. Since it is possible that the standard deviation of a random signal could be proportional to the magnitude of the signal, the existence of a random signal would also explain the slight increase in Figure 3.6. In either case this correspondence between signal magnitude and the ensemble standard deviation is minor, except for the first response; this would imply that the standard deviation of the noise is relatively independent of the signal.

A comparison of autocovariance functions under stimulus and non-stimulus conditions did not reveal any additional information about the dependence of the signal and noise. The variations of this function appeared to depend primarily on the variance, $\sigma_{f}^{2}(t)$, as noted previously (see Figures 4.1, B.6 and B.7). The histogram estimates of amplitude probability density functions under stimulus and nonstimulus conditions were too variable to attempt a comparison (see Figures 4.3 and B.8).

\subsection{Additivity of Signal and Noise}

In the previous chapter the maximum (9.68), minimum (1.03) and average (2.6) rms signal to noise, $\mathrm{S} / \mathrm{N}$, ratios were computed. These 
ratios were calculated from the ratio of the rms value of the EER's in the second interval to the rms value of the ensemble control average in any interval. In addition, the correlation coefficients were found between EER waveforms. Since the $S / N$ ratio estimates the anount of noise remaining in the EER waveforms, a decrease in the $S / N$ ratio should be reflected by a decrease in the correlation coefficients between waveforms. That is, if the signal and noise are additive, then the correlation between EER's should depend on the relative amount of uncorrelated noise and correlated signal contained in these averages.

In Chapter 3 the correlation coefficient between EER waveforms $x$ and $y$ was calculated from the time standard deviations, $\Psi_{x}$ and $\Psi_{y}$, and the time covariance $\Psi_{x y}$, i.e.,

$$
\rho=\frac{\Psi_{x y}}{\Psi_{x} \Psi_{y}}
$$

The $\mathrm{S} / \mathrm{N}$ ratios were found using the equation

$$
S / N=\frac{R_{r m s}}{C_{r m s}}=\frac{\tilde{\Psi}_{S}}{\tilde{\Psi}_{n}}
$$

where $\tilde{\Psi}_{S}$ is an estimate of the average signal time standard deviation and $\tilde{\Psi}_{n}$ is an estimate of the average noise deviation under stimulus conditions (assuming the signal and noise are independent). Both of these functions are dependent on the time variance, $\Psi$, and a mathematical relationship between the two can be established with certain constraints. 
If the EER is composed of signal plus noise, i.e.,

$$
\begin{aligned}
& x=s_{1}+n_{1} \\
& y=s_{2}+n_{2}
\end{aligned}
$$

then the time covariance is

$$
\Psi_{x y}=\psi_{s_{1} s_{2}}-\psi_{s_{1} n_{2}}-\Psi_{s_{2} n_{1}}+\Psi_{n_{1} n_{2}}
$$

If the signal and noise are statistically independent, which insures linear independence, then the two covariances between the signal and noise in Equation 4.18 are zero and Equation 4.14 becomes

$$
\rho=\frac{\Psi_{S_{1} S_{2}}+\Psi_{n_{1} n_{2}}}{\left[\left(\Psi_{S_{1}}+\Psi_{n_{1}}\right)\left(\Psi_{S_{2}}+\Psi_{n_{2}}\right)\right]^{1 / 2}}
$$

If the noise is stationary then the time standard deviation, $\Psi_{n}$, of its estimated average over equal periods of time should be the same, providing the period is sufficiently long. If we assume that the period between stimuli is sufficiently long and that the ensemble average signal is near deterministic for each stimulus, then

$$
\Psi_{S_{1}} \simeq \Psi_{S_{2}}=\Psi_{S}
$$

and

$$
\Psi_{\mathrm{n}_{1}} \simeq \Psi_{\mathrm{n}_{2}}=\Psi_{\mathrm{n}}
$$

The correlation coefficient between EER waveforms can then be written as in Equation 4.22 . 


$$
\rho=\frac{\Psi_{S_{1} S_{2}}+\Psi_{n_{1} n_{2}}}{\Psi_{S}^{2}+\Psi_{n}^{2}}
$$

Which reduces to

$$
\rho=\frac{\rho_{S}(S / N)^{2}+\rho_{n}}{1+(S / N)^{2}}
$$

where $\rho_{\mathrm{s}}$ and $\rho_{\mathrm{n}}$ are the correlation coefficients between the signal and noise and $S / N$ is defined by Equation 4.15 with the $\sim$ deleted. Since we assumed previously that the average signal was near deterministic, $\rho_{s}$ is almost unity. The noise should be incoherent with the stimulus; thus $\rho_{n}$ is near zero and Equation 4.23 reduces to

$$
\rho=\frac{(S / N)^{2}}{1+(S / N)^{2}}
$$

Therefore, if a pair of identical signals are added to stationary noise, which is independent from the signal, then the correlation coefficient between the resultant waveforms can be calculated from the $\mathrm{S} / \mathrm{N}$ ratio.

Using the three $S / N$ ratios given on page 59 , the following values of $\rho$ are found from Equation 4.24 :

$$
\begin{aligned}
& \rho_{\text {max }}=0.989 \\
& \rho_{\text {min }}=0.515 \\
& \rho_{\text {ave }}=0.871
\end{aligned}
$$

Therefore, subject to the above stipulations, one would predict the above values for correlation coefficients between the EER responses 
in the second interval. A comparison of these predicted correlations with those calculated in the previous chapter, i.e.,

$$
\begin{aligned}
& \rho_{\text {max }}=0.99 \\
& \rho_{\text {min }}=0.53 \\
& \rho_{\text {ave }}=0.88
\end{aligned}
$$

discloses that the former and latter are nearly equivalent.

The implications of this strong similarity between the predicted and measured coefficients are two-fold. First, the $\mathrm{R}_{\mathrm{rms}}$ to $\mathrm{C}_{\mathrm{rms}}$ signal to noise ratio provides a good estimate of the background activity remaining in the EER. Second, the assumption of an additive signal to noise is further supported. One argument which might be presented against the validity of the predicted coefficients is that the assumptions of $\rho_{\mathrm{n}}=0$ and $\rho_{\mathrm{s}}=1.0$ are not realistic. However, the average correlation coefficients calculated across segments of the control waveforms were very near zero. This would partially reject the argument, particularly in the case of the average predicted correlation coefficient.

Arother method to investigate the additivity of the signal and noise is to observe the growth of the signal to noise ratio with the number of averages. For example, if a near deterministic signal and random background noise are additive and independent, then the waveform of the average signal will remain essentially constant and the variance of the average noise wili decrease directly with the number of sumnations in the average. If we assume that the noise is stationary, then the true noise mean is a constant, which in the 
present study is preset to zero. The rms value of the average noise will approach this zero value as the number of summations, $N$, becomes very large (noting that the variance of this average about the zero mean decreases by a factor of $\frac{1}{N}$ ). The rms value of the average signal should be relatively invariant with the number $N$. Therefore the signal to noise ratio, $\mathrm{S} / \mathrm{N}$, should be expected to increase as the number of summations in the average increases.

The minimum rate of the $S / N$ ratio increase can be specified in terms of the true ensemble variance, $\sigma_{f}^{2}(t)$, and a probability determined from the Tchebycheff inequality (see Equation 4.3). Thus, we should expect the estimated $S / N$ ratio to reflect a random increasing trend with the number of summations in the average. However, how can this increase be examined? The technique chosen to observe this growth was to calculate the ms value (in the 60 to $300 \mathrm{millisecond}$ region) of the EER at every even number of summations and to compare this value to the rms value of the \pm average of the same data. After a number of summations, the \pm average of the data should provide an amplitude estimate for the background noise and the EER a signal amplitude estimate. The $S / N$ ratio can then be formed by dividing the EER by the \pm average at every even number of summations, $N$, within the average. This procedure was repeated for each set of data following the ten selected stimuli.

A plot of the $S / N$ ratio versus the number of summations $N$ for the EER's to the first five stimuli is provided in Figure 4.4. Although the $S / N$ ratio demonstrates an overall slight increase with $N$ in each 


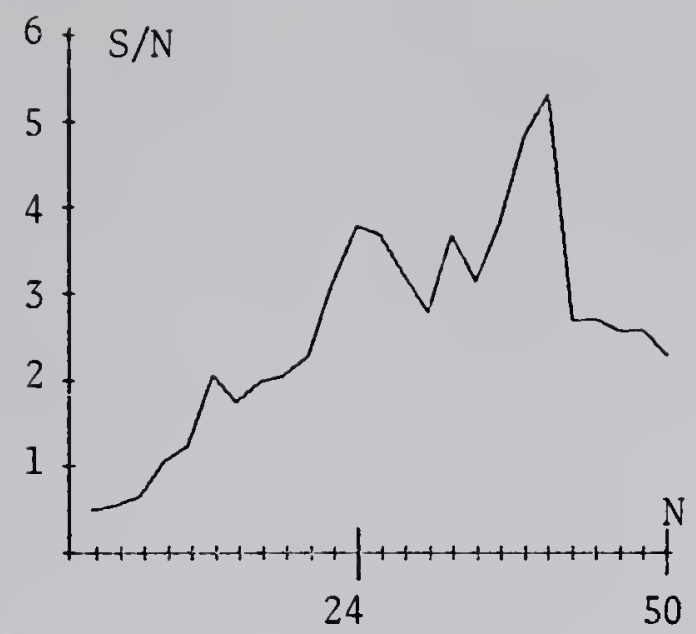

Response 1
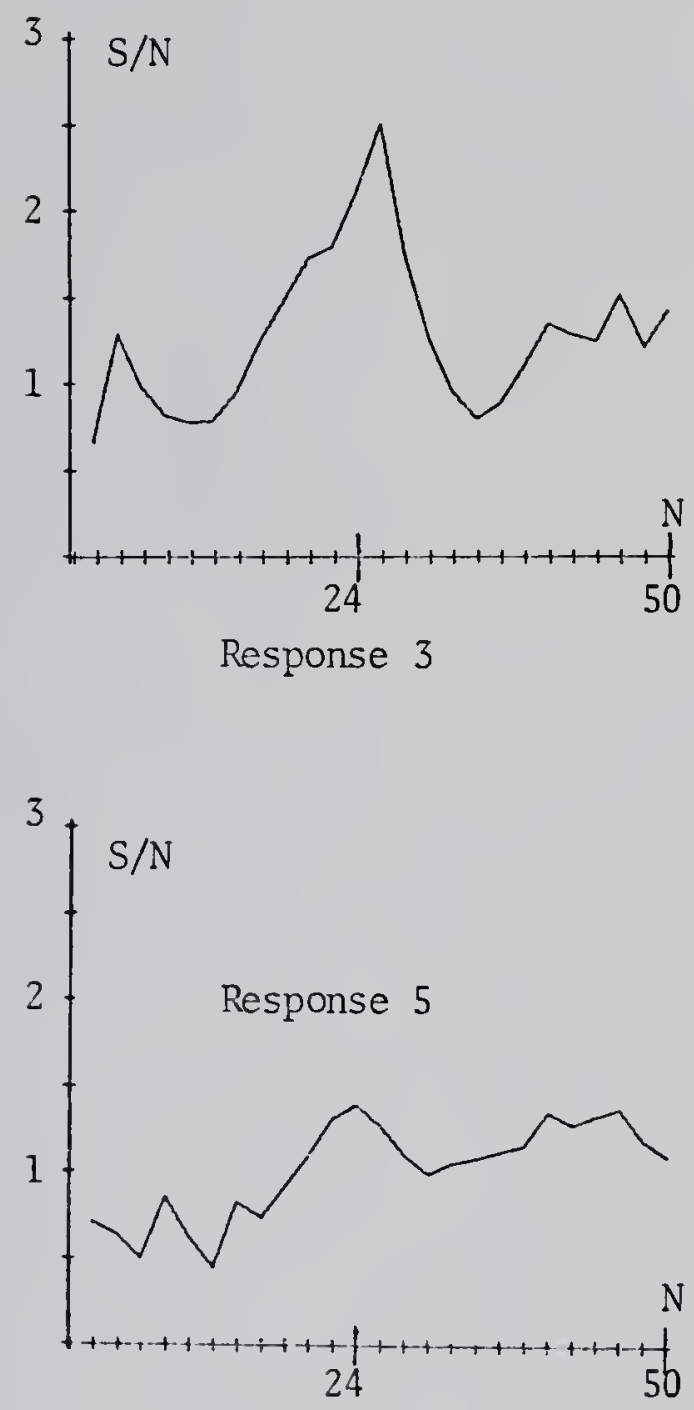

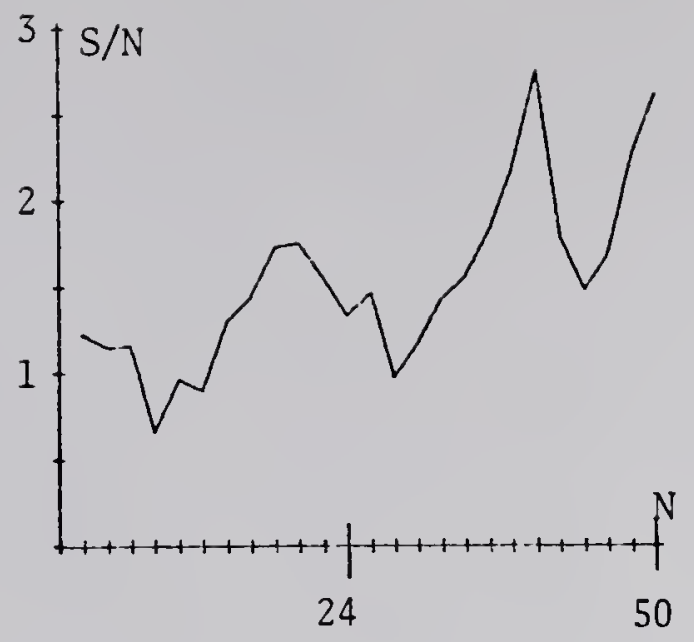

Response 2

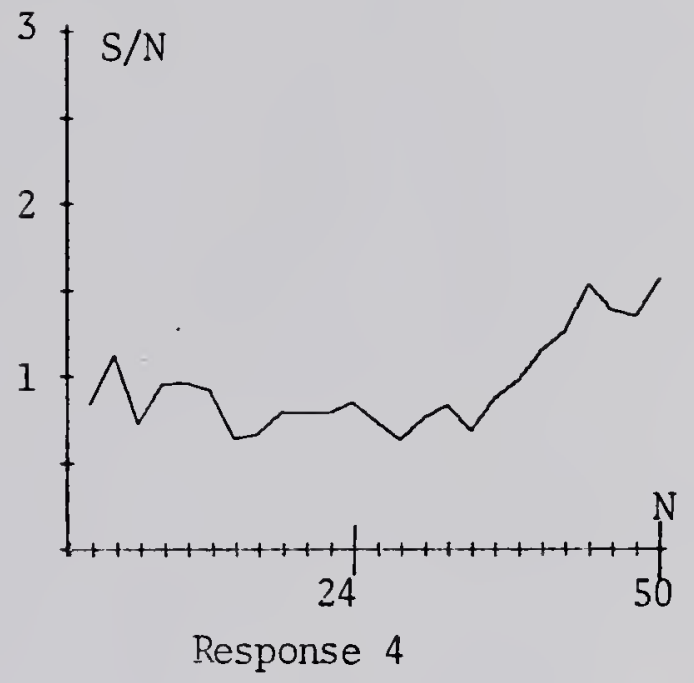

FIGURE 4.4

S/N Ratios Versus Number of

Summations

S/N Ratios for the EER are calculated at every even number of summations, $\mathrm{N}$. 
of the plots in this figure, the random fluctuations of $\mathrm{S} / \mathrm{N}$ overshadow this growth. In addition, it should be noted that the $\mathrm{S} / \mathrm{N}$ ratio fluctuations of the first response are nearly twice those of the remaining responses. In a similar plot of the $\mathrm{S} / \mathrm{N}$ ratio for EER's to later stimuli (see Figure B.9), an overall decrease in this ratio with $\mathrm{N}$ can be noted. These plots appear to discredit the signal plus noise assumption. However, this conclusion contradicts the previous results in this chapter and questions the results achieved by the established technique of response averaging. Therefore, the adequacy of the above test becomes highly suspect.

The inadequacy of this test appears to be the result of: (1) the large variations in amplitude of individual EEG's which appear to introduce abrupt changes in the noise estimates; and (2) the $\mathrm{S} / \mathrm{N}$ ratio estimate being probably inaccurate except for a large number of sums (i.e., EER is more noise than signal). The first shortcoming might be eliminated by performing the same test on a single subject (i.e., using sequential as opposed to ensemble averaging); the latter shortcoming could be reduced by beginning with forty to fifty sums and increasing the number of summations. Still another technique would be to utilize Equation 4.24 and discuss the improvement in $\mathrm{S} / \mathrm{N}$ by the degree of correlation of the average after $\mathrm{N}$ sumuations with a preset response template or norm. This correlation technique would be more sensitive to the waveshape of the response, which would help to separate signal and noise energy in the case of fewer summations. 


\subsection{Summary}

The separation of the foregoing examinations into three groups is somewhat artificial because of the inter-dependence of the results obtained. Therefore, many of the results obtained actually provide tests for more than a single assumption. The above examinations are grouped into three areas to help organize the test results and produce a more readable text.

The results of these tests strongly imply that the mean of the background noise is time invariant and that its amplitude is relatively independent of the signal activity. The higher order statistics (i.e., standard deviation, autocovariance and pdf) of the noise appear to be non-stationary. However, the time fluctuations of the estimates of these higher order statistics are dependent on the variance of the estimation process, which was not investigated. The average and peak-to-peak fluctuations of the ensemble standard deviations under stimulus and non-stimulus conditions were very similar, which further indicates that the signal is independent of the noise. The average correlation coefficients of a theoretical signal plus noise were nearly identical to those calculated from the actual averaged responses. Thus, the assumption of signal plus noise was partially supported. However, a subsequent test of the theoretical increase in the $\mathrm{S} / \mathrm{N}$ ratio by averaging signal plus noise data provided inconcIusive results. 


\section{CHAPTER 5}

\section{SUMMARY}

To date, most investigations of evoked potentials monitored at the human scalp have utilized the average of a series of responses recorded from a single subject to define components of the evoked response. This technique has provided a fairly consistent estimate of an individual's average ER. However, with this method it is not uncormon tc obtain quite dissimilar averages from different subjects under identical experimental conditions. This contrast in the ER estimates across a population has hindered the interpretation of the effects of stimulus parameters on the ER and frustrated the attempt to use this average for clinical diagnosis. It may be possible that the character of an ER is, like a signature, unique for each subject. If this analogy is valid, utilization of the ER as a research or diagnostic tool might be limited. However, if one could establish a particular feature of ER estimates common to a considered normal subject population, then this template or norm might identify that quality associated with a population of responses evoked by a specific stimulus. This would be analogous to identifying the characteristics attributed to a specific letter of the alphabet in a set of signatures. The influence of variations in stimulus parameters and physiological abnomalities might then be recognizable in the degree of deviation of a subject's ER estinate about this norm. 
The present study investigated one method of estimating such a response norm. The estimation technique utilized was an average across an ensemble of subject EEG's containing evoked activity. The advantages of this method were two-fold. First, it permitted estimation of conmon inter-subject response activity evoked by a single stimulus. Second, the collection of subject EEG's strongly resembled an ensemble of independent sample functions from a stochastic process. This second advantage permitted an investigation of several prevalent assurmptions about the statistical nature of the evoked signal and background noise.

The ensemble average revealed a fairly consistent, recurring component complex following each of a series of photic stimuli, i.e., tein stimuli chosen between the first and fiftieth stimuli in a periodic stimulus train. This averaged complex or primary response mainly consisted of a positive to negative to positive potential, near sinusoidal in form, which began approximately 60 milliseconds after the onset of each stimulus. Significant correlation coefficients were found between all pairings of the ten ensemble averages in this primary region. Outside of this region most of the paired correlations between waveforms were insignificant. The average of these paired correlations provided a reliable measure of the conformity across segments of the ten responses. Control estimates of the amount of background noise contained in the primary response region preaicted that this complex could contain from 10 to 50 per cent noise, with an expected amount of 28 per cent. 
Under the assumption that the evoked signal and background noise are additive, this prediction was further substantiated by the range of the correlation coefficients between paired response estimates. Therefore, these results implied that the control estimate provided a good evaluation of the amount of distorting noise remaining in the ensemble evoked response, EER. Since the primary response was detectable in each EER, these results also imply that even with a signal to noise ratio of unity the average response is observable.

The ensemble average technique also provided an estimate of the changes in the evoked response with the nunber of stimulus repetitions. That is, each EER was the average of the activity attending a single stimulus. An examination of the changes in the response estimates following each stimuli disclosed moderate component fluctuations in amplitude and latency of the primary response. Since these fluctuations did not exhibit any specific trends or transient variations with the number of stimulus repetitions, they appeared to be the result of background noise disturtion rather than response changes. The one noted exception, the uniqueness of the EER to the first flash, is hypothesized to be the result of intersubject response variability or some initialization of the visual system. This first response estimate appeared to contain a low frequency component which was not evident in the remaining estimates. In addition, the time fluctuations of the ensemble standard deviation about the ensenile average resembled the waveshape of this average for the first 450 milliseconds following the first stimulus. This 
was a characteristic relatively unique to the standard deviation attending the first stimulus and resulted in a decrease in the confidence of the first EER. Another singular property of the potentials attending the first stimulus was demonstrated in plots of the EER signal to noise ratio as a function of the number of subjects in the average. The magnitude of the variations of the $\mathrm{S} / \mathrm{N}$ ratio in the plot for the first response was nearly twice that found in the remaining graphs. Thus, these graphs reflected the greater variability of the initial potentials. Because of these unique properties of the first response estimate it may be advisable to delete this response when comparing VER's across subjects.

The average of the ten EER's waveform appeared to provide a reliable, relatively noise-free response template. In this template the primary sinusoidal complex was enhanced and the amplitudes of the remaining components were reduced. The character of these reduced components strongly resembled that of a similar single control template. Thus, this response template further supported the hypothesj.s that the comnon inter-subject response activity generally is restricted to the primary response region. This average response template also implied that investigations which compare the sequential average obtained from different subjects should possibly restrict this comparison to the 60 to 300 milliseocnd region after stimulus onset. However, since the above template would not extract intersubject signal components whose latency varies from one subject to the next, this restriction might be inappropriate for some studies. 
Three conmon assumptions about the statistical character of the signal and noise necessary to theoretically justify response averaging were examined using the ensemble of subject EEG's. The results of these examinations generally supported the assumptions that: (1) the noise mean is stationary; (2) the signal and noise are independent; and (3) scalp potentials consist of signal plus noise. The first assumption was investigated by testing two amplitude estimates of the average background activity against a hypothesized stationary mean. Of the twenty times tested, the stationary hypothesis was rejected only twice. The conclusiveness of these tests was dependent on the second assumption, i.e., the independence of signal and noise. The combination of signal and noise activity is demonstrated to be generally non-stationary. If the signal and noise are dependent, the hypothesis tests on the \pm average should demonstrate this dependence by rejecting the stationary hypothesis. Since this was not the case, the results of the hypothesis tests appear to definitely support the assumption of a stationary noise mean. An investigation of the stationarity of the higher noise moments was generally unsuccessful. Since the variance of the estimate of these statistics was unknown, the time dependence of these functions could not be evaluated. However, the relative degree of the time fluctuations of the variance, autocovariance, and pdf functions implied that the higher moments of the noise might be non-stationary.

An investigation of the second assumption, the independence of signal and noise, was accomplished by comparing the ensemble \pm average 
and standard deviation under stimulus and non-stimulus conditions. The \pm average provided an estimate of the noise amplitude with and without the evoked activity. The range of amplitude fluctuations of this average was nearly equal under both conditions. The average and peak-to-peak fluctuations of the ensemble standard deviation under stimulus and non-stimulus conditions were very similar. Therefore, both the noise average and the deviation about this average appear to be independent of the signal activity.

An examination of the third assumption, additivity of signal and noise, was generally hindered by the inability to separate these two components. This shortcoming thwarted an attempt to empirically demonstrate the theoretical improvement of the signal to noise ratio by averaging the assumed signal plus noise potentials. However, the relative stability of the average estimates and the previous prediction of the signal-noise ratio by the correlation coefficient between waveforms supported this signal plus noise assumption.

The results of this investigation of an ensemble of subject EEG's which contained evoked responses have provided an estimate of the common inter-subject response. The estimated response template found in this study was limited to responses evoked by visual stimuli, specifically a monocular, periodic flash of light. The applicability of this template to other studies of responses in this modality is at present uncertain. However, the method utilized in this investigation has provided at least one technique for obtaining an inter-subject response norm to a specific stimulus. It is impractical 
to utilize this ensemble technique in most experinental or clinical investigations because of the large subject population required. Therefore, the applicability of the technique is restricted to isolated studies. The results achieved in such studies, however, should be useful in many other investigations which compare the intrasubject response average across a subject population.

The ensemble of subject EEG's appears to provide a worthwile tool by which to evaluate theoretical assumptions about the statistics of the EEG. However, the degree of sample variation noted in this study seems to indicate that a greater number of sample functions would provide a more reliable evaluation. This is particularly true in the case of the estimation of the higher ensemble moments and the ensemble probability density function. In order to obtain a significant improvement, a fair amount of increase in the number of sample functions would be necessary. The advantages of such an increase would probably be overshadowed by the difficulties presented in acquisition of this larger ensemble. 
APPENDIX A

EXPERIMENTAL PROCEDURE

\section{A.1 Data Acquisition}

The data used in this experiment was collected from fifty subjects ( 52 males, 18 fernales). The right eye acuities of all subjects were normal or correctable to normal as measured on a Snellen chart. Subjects were asked their hand preference and given a far vision sighting test for eye dominance. One pair of bipolar electrodes (Lexington C-100) were placed $5 \mathrm{~cm}$ apart on the scalp, located vertically on the occiput midline with the lower electrode over the inion. This configuration was chosen becuase it has been noted to produce more reliable VER's across subject populations. Each subject was seated upright in an electrically shielded Faraday cage and asked to concentrate on the light seen through the right eye-piece of a modified troposcope. During a stimulus (signal) trial the light consisted of a xenon $\mathrm{flash}$ of intensity $3.6 \mathrm{log} \mathrm{ft}$-lamberts subtending $2^{\circ} 15^{\prime}$ in an invariant incandescent surround of intensity $1.310 \mathrm{ft}$-lamberts subtending $18^{\circ} 24^{\prime}$. The flash was presented periodically (approximately $0.9 \mathrm{~Hz}$ ) in two trials and aperiodically (average rate of approximately $0.4 \mathrm{~Hz}$ ) in a third. Each signal trial was followed by a control (noise) trial identical to the preceding stimulus trial with the xenon flash occluded. After each trial the subject was permitted a rest period of two to three minutes; total time required of each 
subject was usually less than forty-five minutes.

The potential difference monitored between the scalp electrodes was fed simultaneously through two Grass P5111 differential amplifiers (channels 1 and 2) with a gain of $100 \mathrm{~K}$ and bandpass of 0.3 to $50 \mathrm{~Hz}$. The bandpass of the signal in channel 2 was further restricted by a Khron-Hite $330 \mathrm{~B}$ electronic filter from 0.3 to $15 \mathrm{~Hz}$. These amplified signals were then fed into a Sanborn 2000 FM magnetic tape recorder and a Mnemotron $400 \mathrm{C}$ computer of average transients (CAT). Also recorded on magnetic tape was the output of a Grass S-8 stimulator which provided an external trigger for the CAT ard a Grass PS-2 photostimulator which powered the xenon flash. In order to detect changes in equipment gain from one subject to the next a calibrate signal from a Medistor C-1A Calibrator was processed in a manner similar to EEG data prior to the experimental run for each subject.

\section{A.2 Data Processing}

In each trial the CAT sequentially summed each one second epoch of the bioelectric potentials following each stimulus trigger. In this manner VER and control waveforms were provided for each subject. These waveforms were permanently registered on a Sanborn 701A Strip Chart Recorder. From these records the "best" signal and noise trials (under periodic stimulus conditions) were chosen. Judgment of "best" was obtained strictly on an amplitude basis, disregarding the waveshape of the sunmed response to avoid biased results. That is, the larger sequential averaged signal and smaller sequential averaged noise amplitudes were judged to be the best. 
The unprocessed analog signals from these better trials were then digitized and stored on a memory disc of an IBM 1800 computer. Since the disc could only accomodate a total of approximately 4.4 $\left(10^{5}\right)$ data words, it became necessary to limit the amount of analog data stored. Therefore, the storage of analog potentials (digitized at a rate of 200 samples per second) was restricted to ten near one second epochs of data for each subject and each channel. The ten epochs chosen followed the first through fifth, tenth, twentieth, thirtieth, fortieth and fiftieth stimuli in the periodic stimulus train. These ten epochs were chosen to provide a measure of both the initial (first few seconds) and prolonged (forty to fifty seconds) changes in the evoked activity with repetitions of the stimulus. Each epoch consisted of the first 190 digitized points following each of the ten stimulus triggers; thus a single epoch is 950 miliiseconds in duration.

In order to eliminate any near $\mathrm{DC}$ potentials introduced by equipment base line shifts, the mean was removed from each 190 point epoch. Equipment gain fluctuations across the subject population were also eliminated by multiplying the digitized potentials by a constant inversely proportional to the magnitude of the recorded calibrate signal. Computations on this adjusted array of data provided most of the results contained in this study. The programs utilized to obtain the data were fairly simple in nature and are therefore omitted from the text. The numerical results of these computations were converted to graphical displays by a Conputer Industries 135 On-Line Incremental Plotter. 
APPENDIX B

SUPPLEMIENTARY FIGURES AND TABLES 


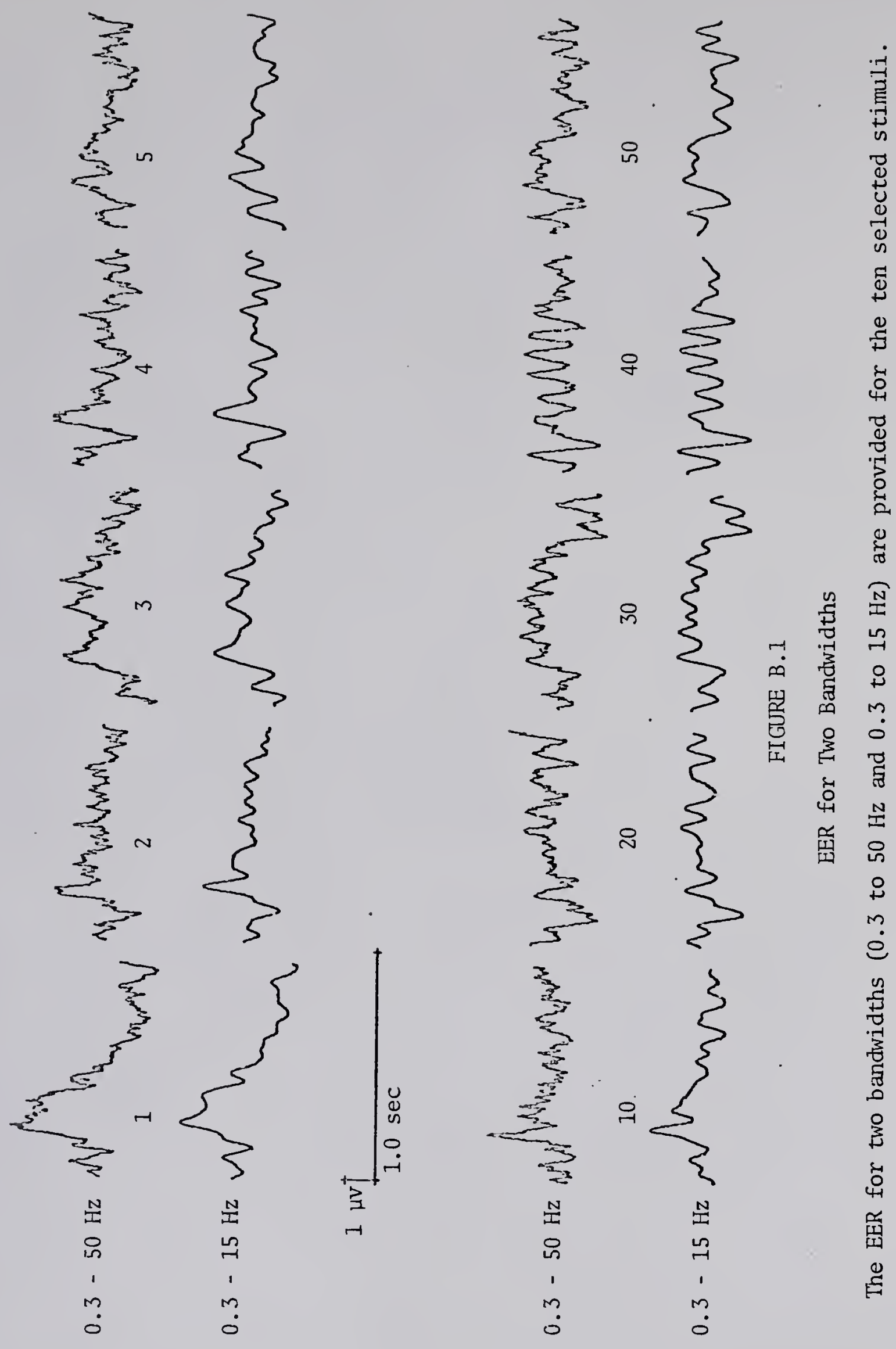



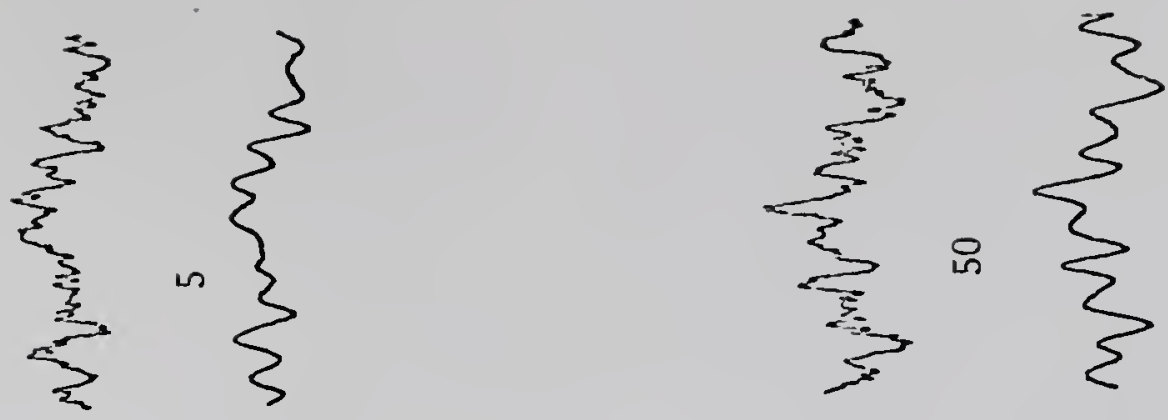

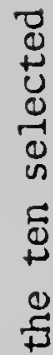
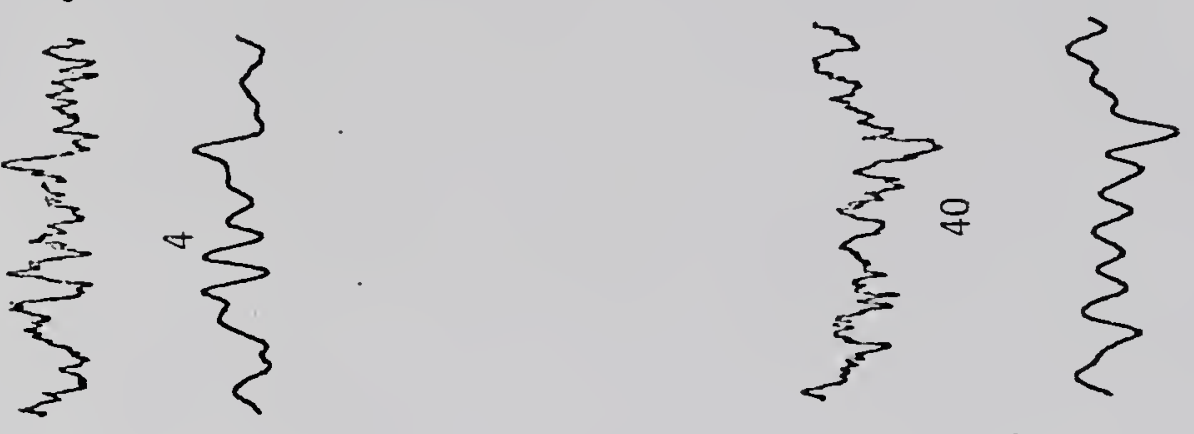

总
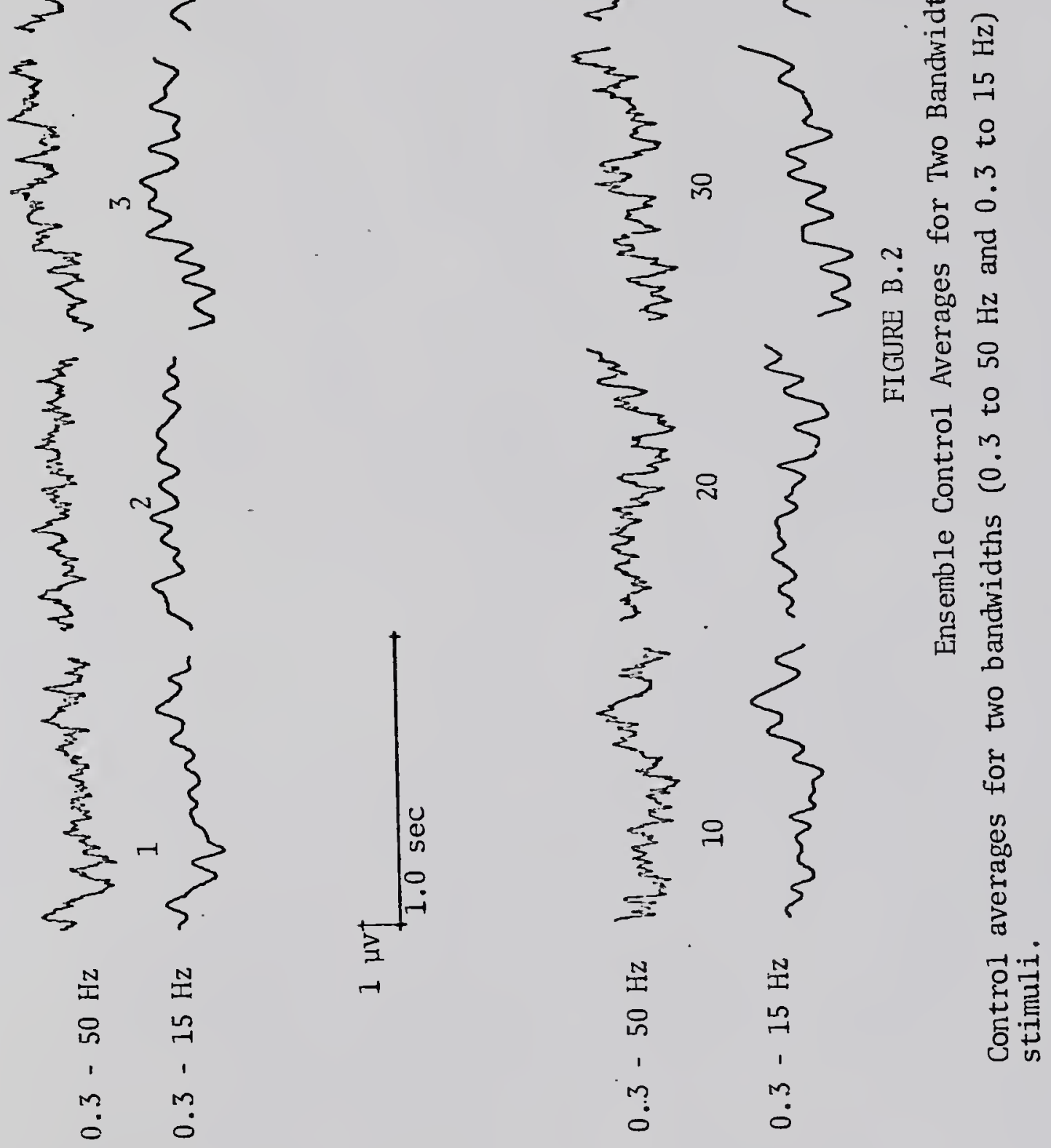

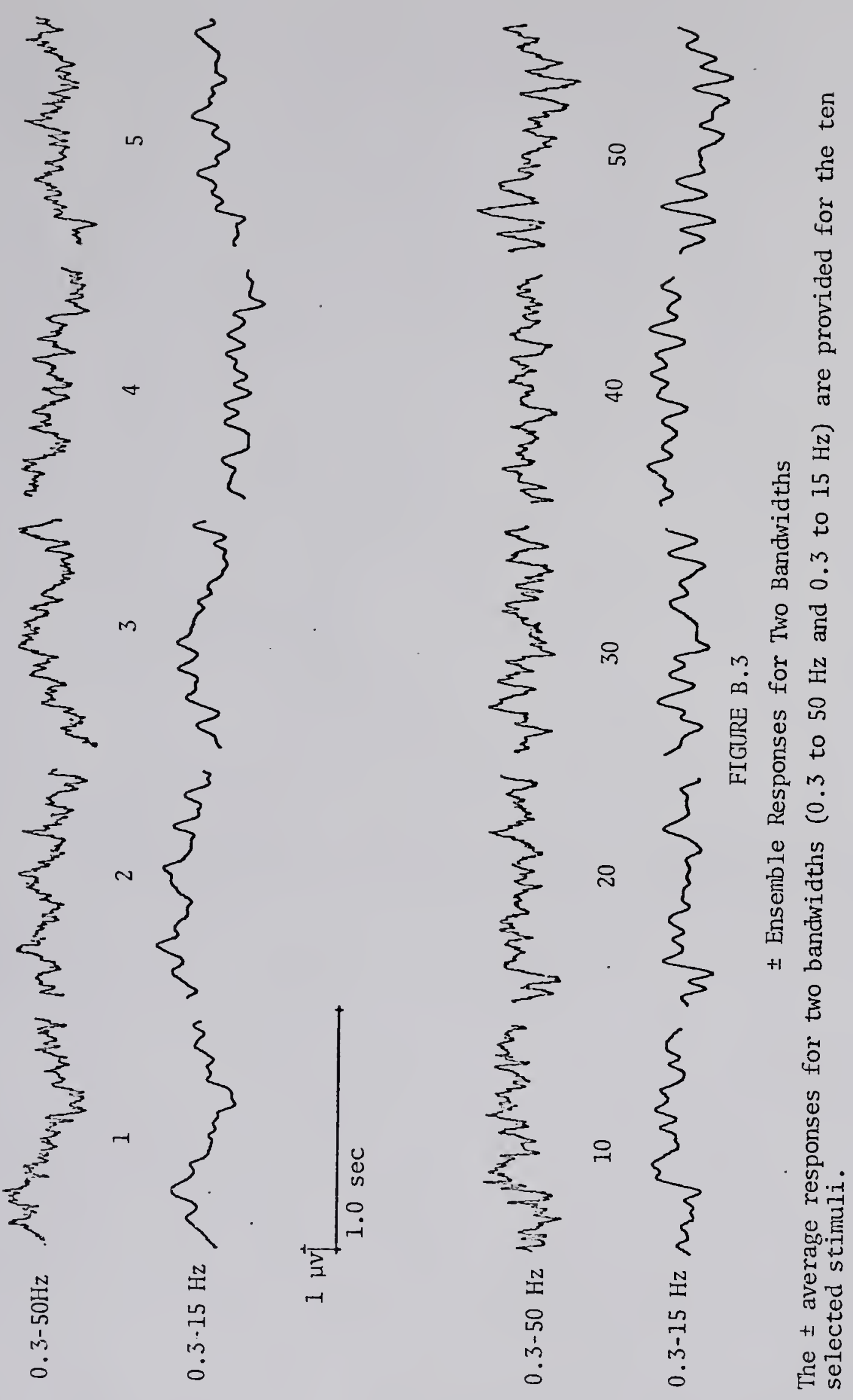

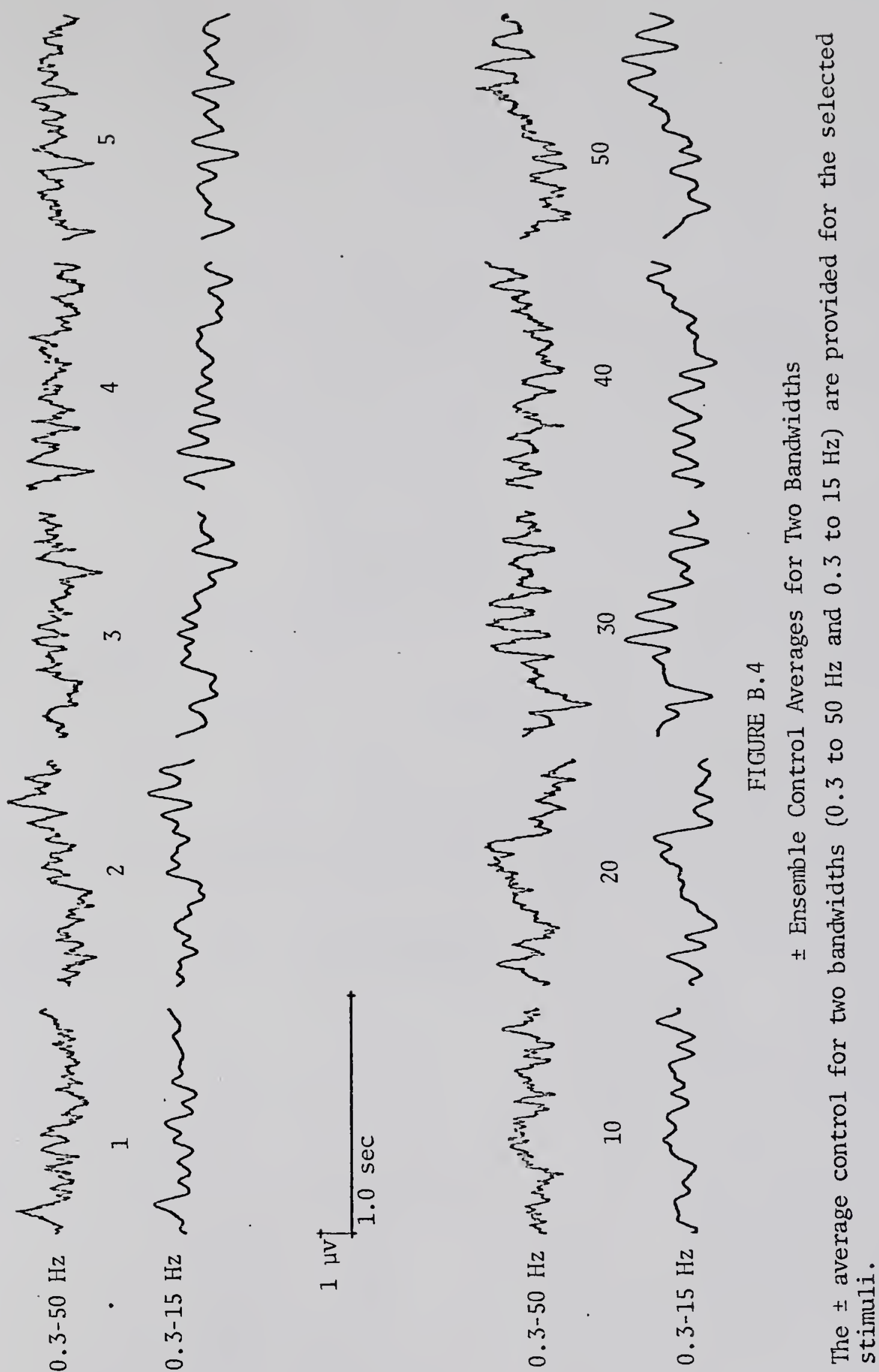


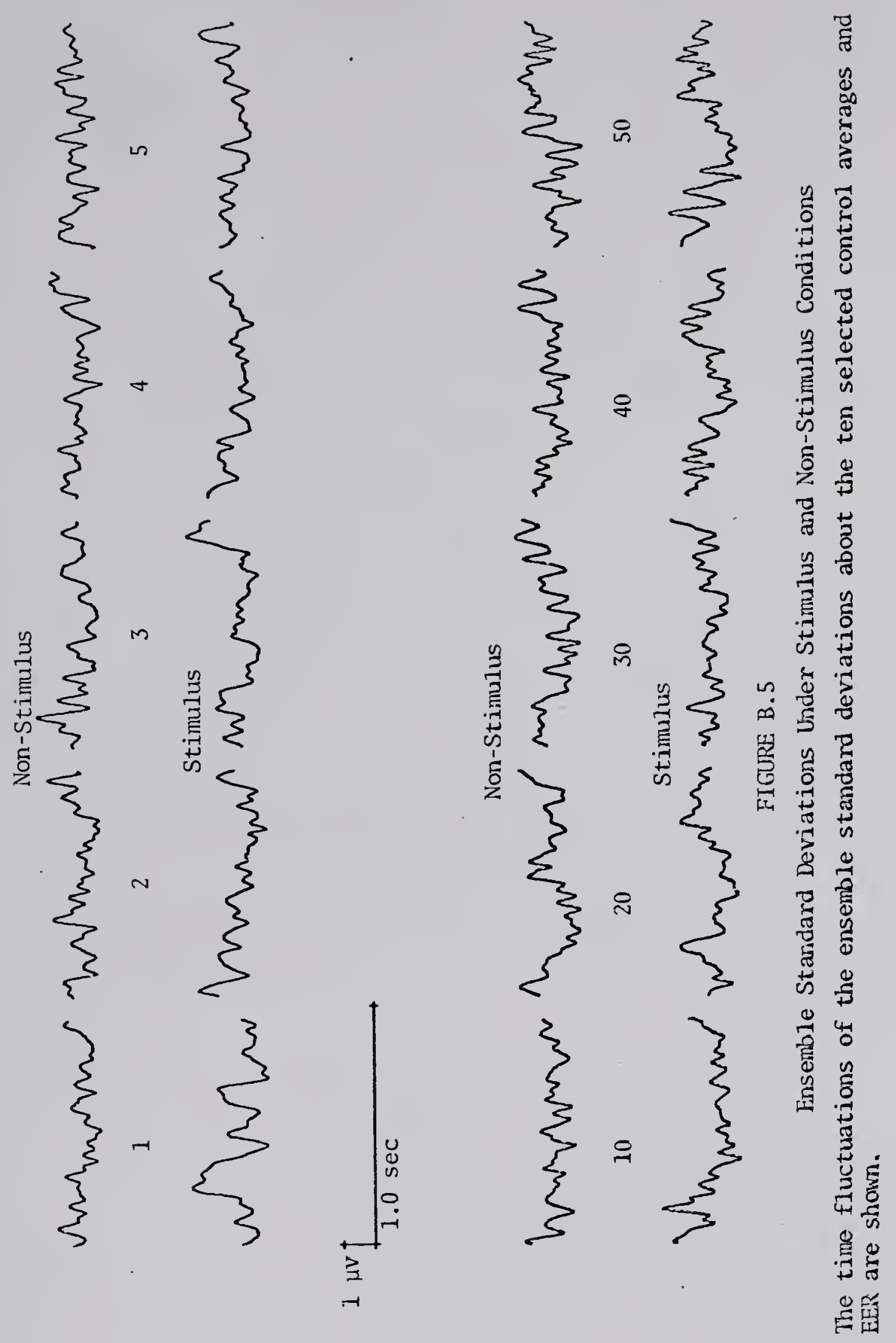




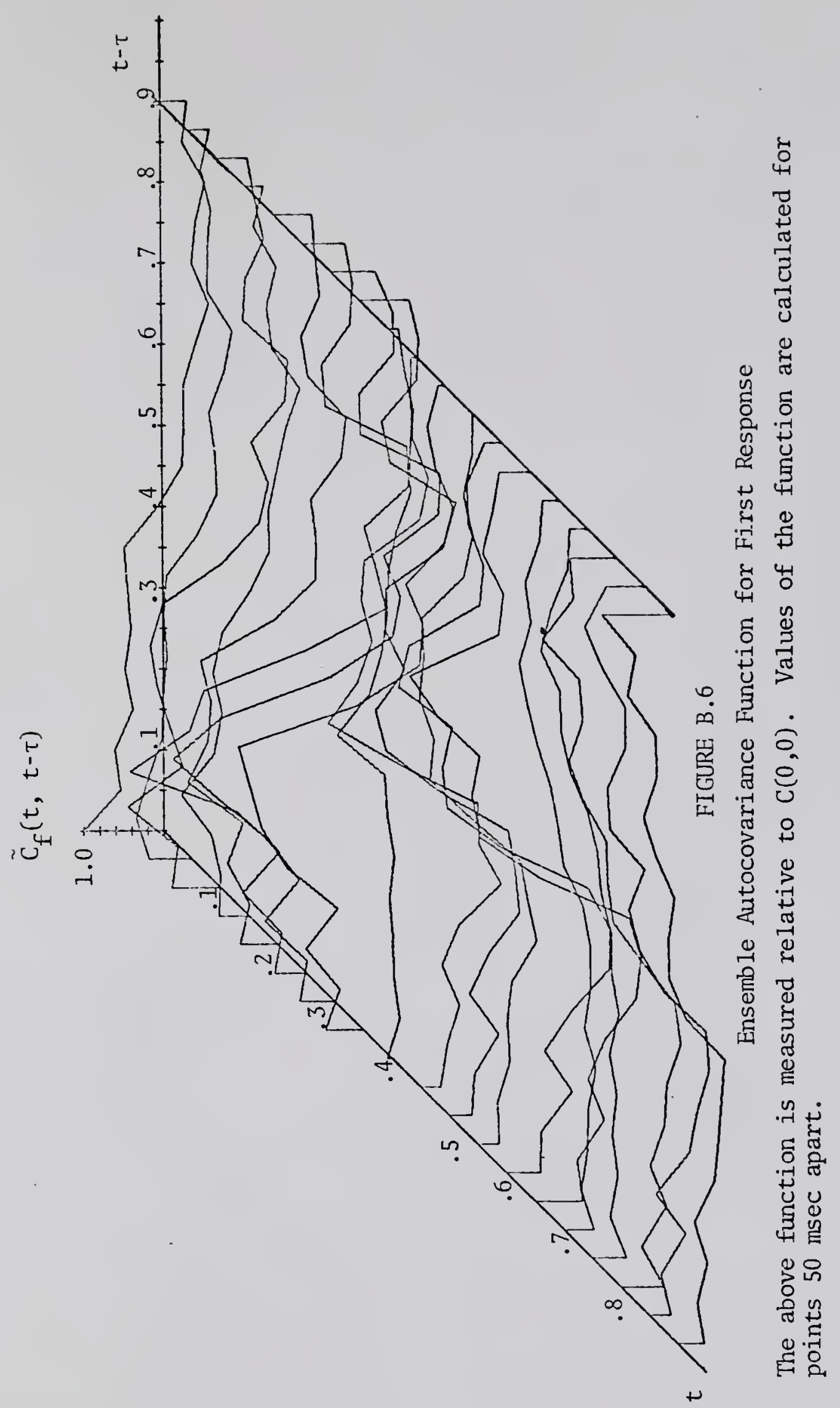




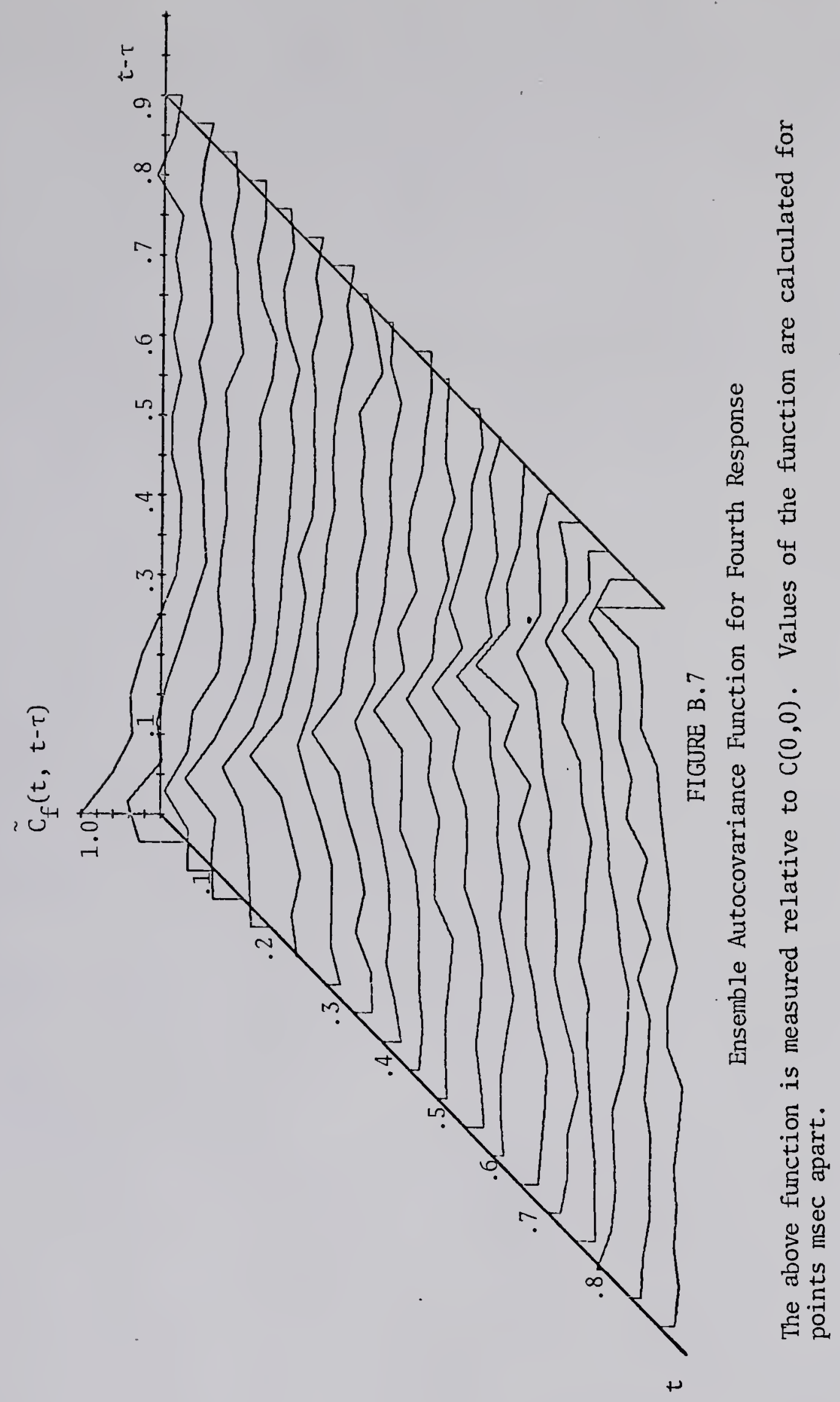




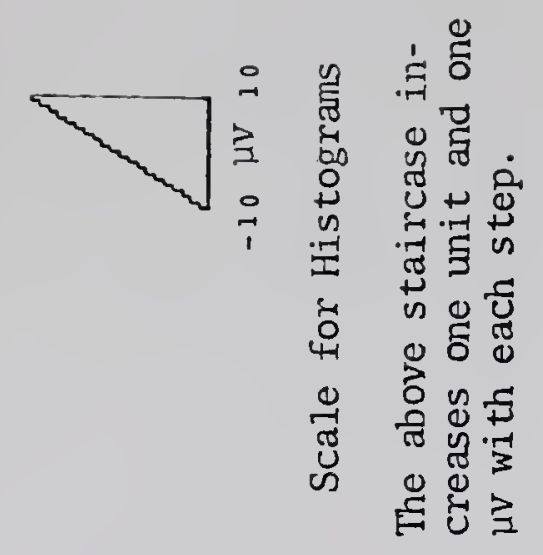

हु-

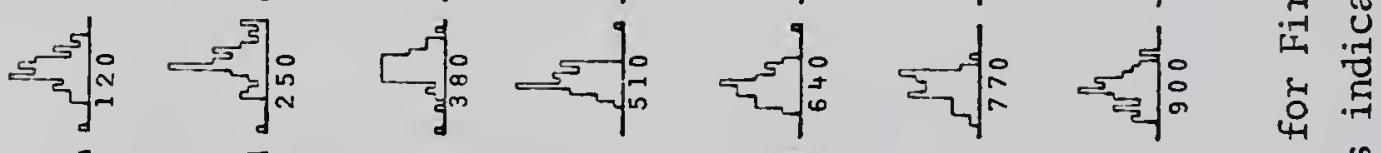

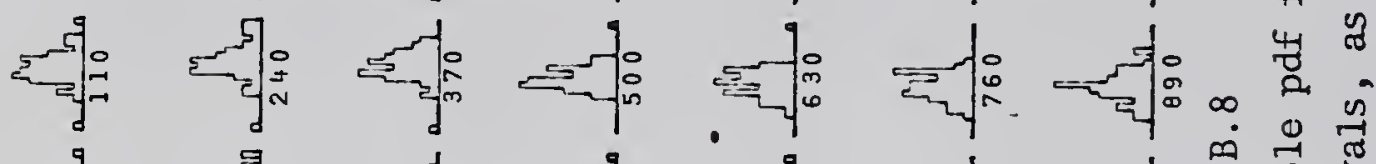

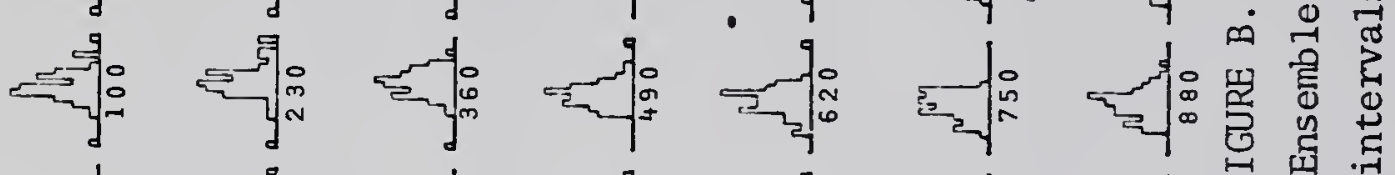

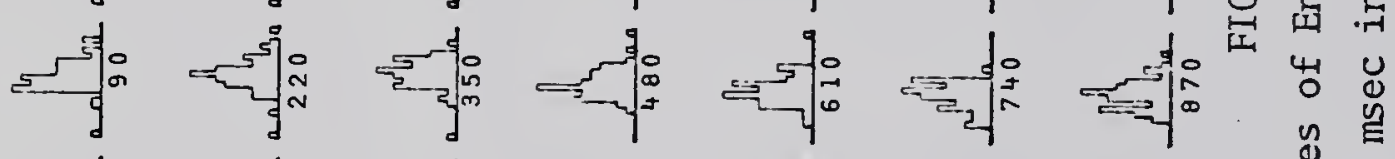

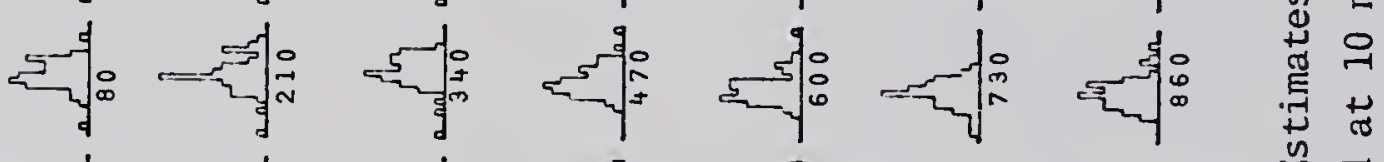

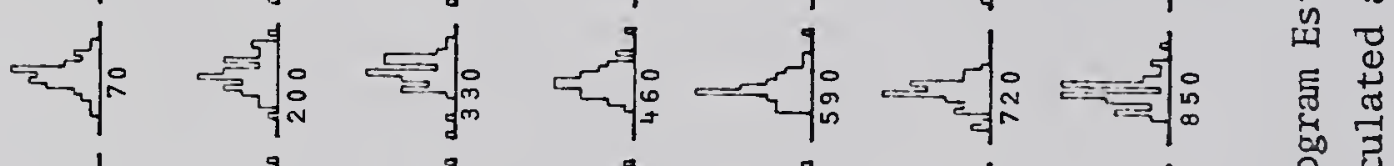

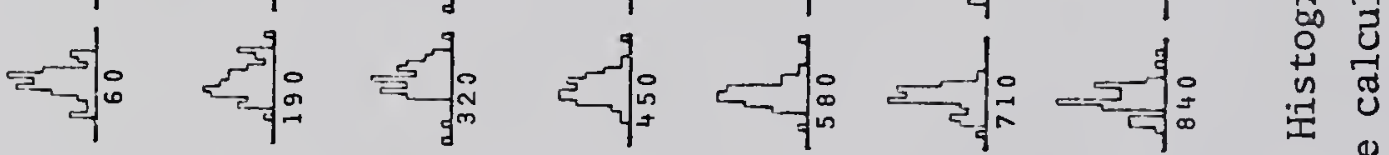
to $)_{0}$

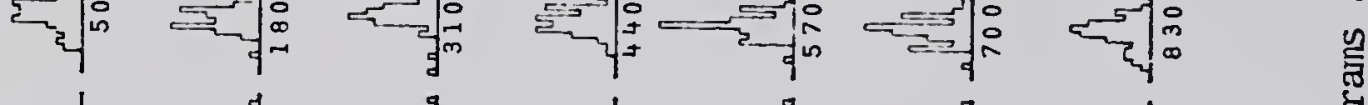

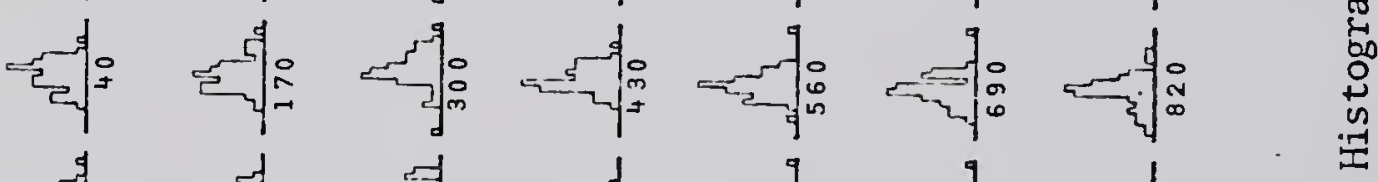

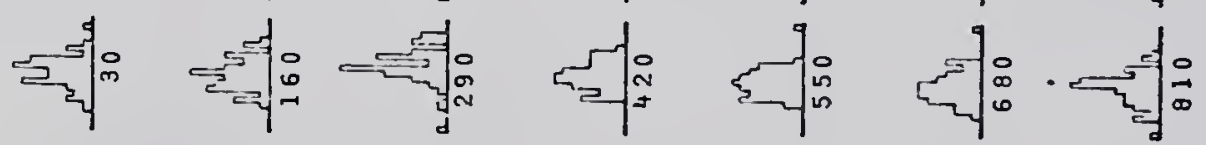

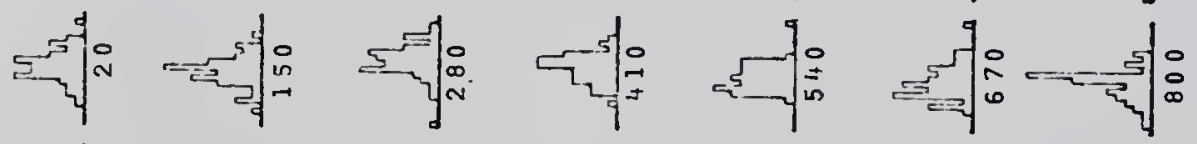

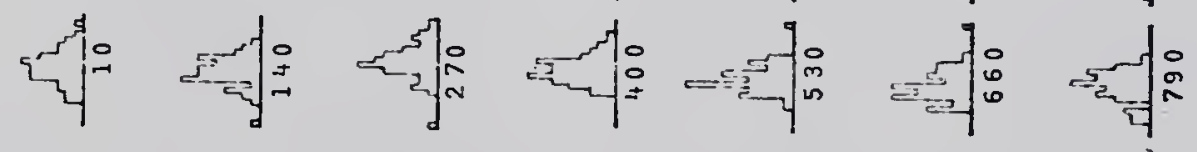



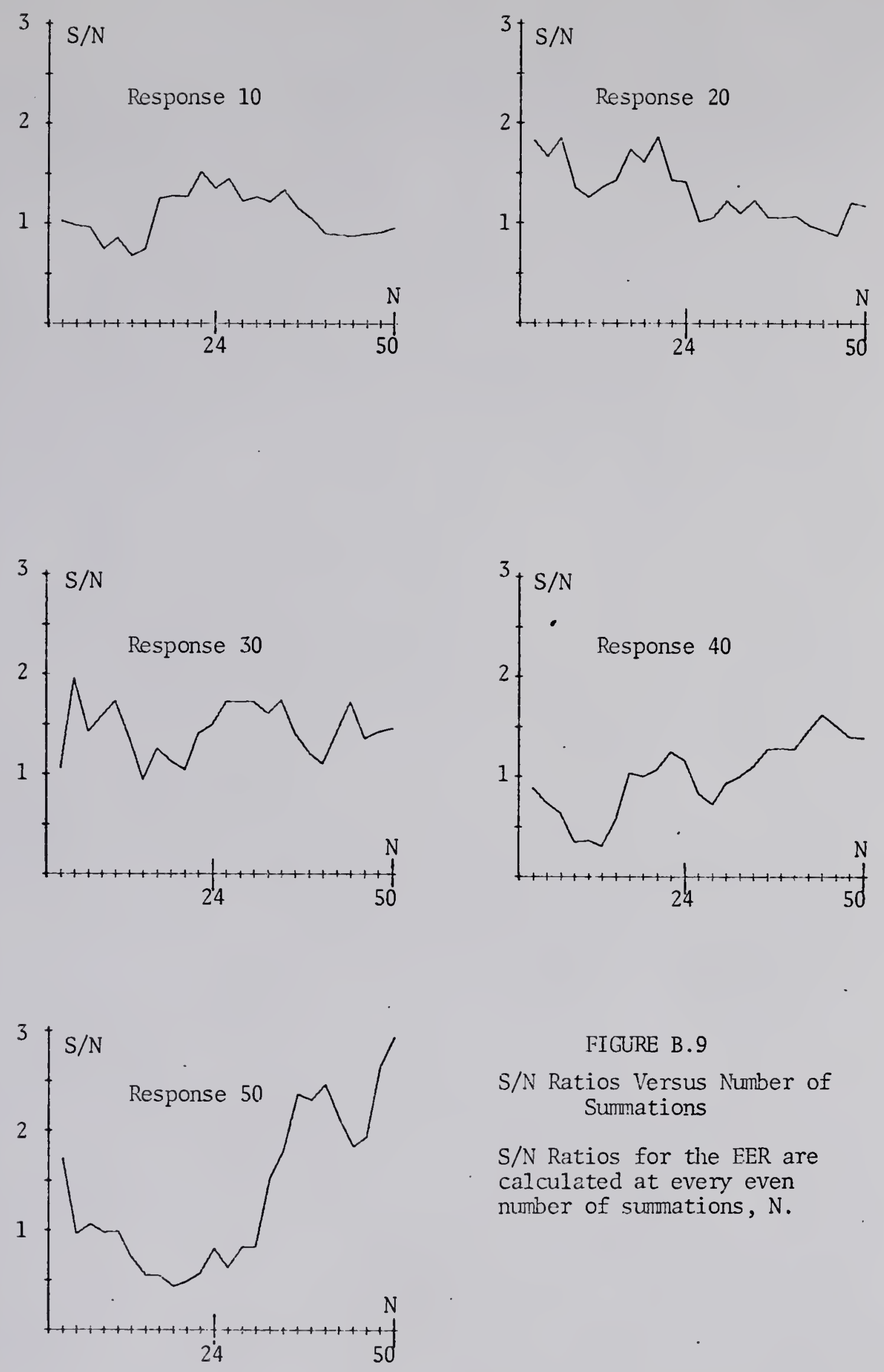

FIGURE B.9

S/N Ratios Versus Number of Summations

S/N Ratios for the EER are calculated at every even number of summations, $\mathrm{N}$. 


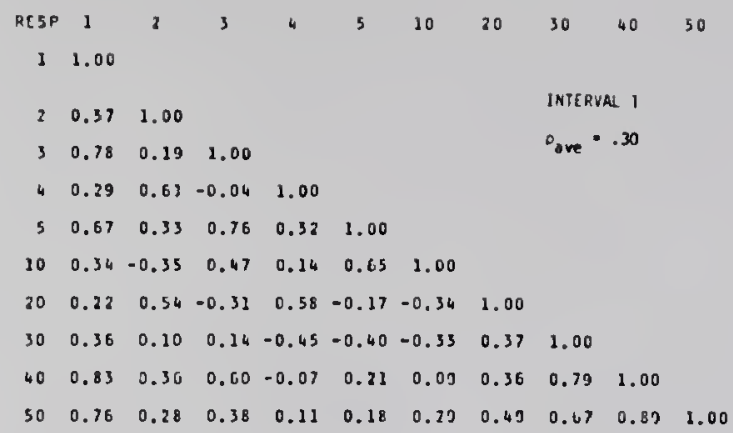

\begin{tabular}{|c|c|c|c|c|c|c|c|c|c|c|}
\hline QESP & 1 & 2 & 3 & 4 & 5 & 10 & 20 & 30 & 40 & 50 \\
\hline 1 & 1.03 & & & & & & & & & \\
\hline 2 & -0.10 & 1.00 & & & & & & INTERVAL & L. 3 & \\
\hline 3 & 0.45 & -0.55 & 1.00 & & & & & Dave" & .12 & \\
\hline 4 & 0.88 & 0.25 & 0.38 & 1.00 & & & & & & \\
\hline 5 & -0.49 & 0.74 & -0.71 & -0.25 & 1.00 & & & & & \\
\hline 10 & -0.15 & 0.53 & -0.21 & -0.05 & 0.68 & 1.00 & & & & \\
\hline 20 & 0.12 & 0.53 & -0.40 & 0.30 & 0.19 & -0.35 & 1.07 & & & - \\
\hline 30 & 0.01 & 0.74 & -0.47 & 0.29 & 0.78 & 0.48 & 0.50 & 1.00 & & \\
\hline 40 & 0.53 & 0.60 & -0.00 & 0.73 & 0.33 & 0.23 & 0.58 & 0.91 & 1.00 & \\
\hline so & -0.48 & 0.11 & -0.48 & -0.54 & 0.02 & -0.03 & 0.05 & -3.44 & -0.62 & \\
\hline
\end{tabular}

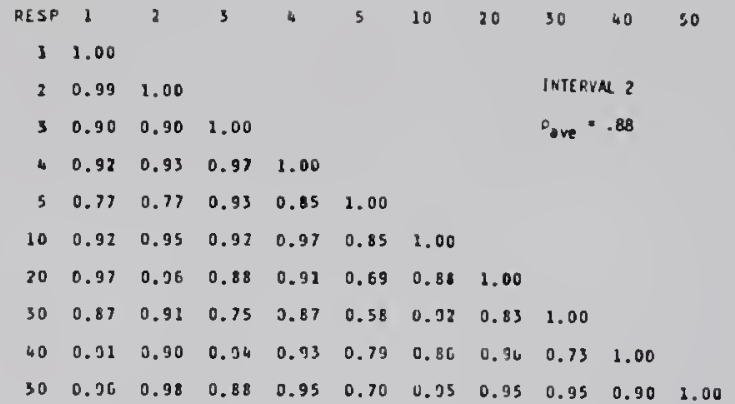

\begin{tabular}{|c|c|c|c|c|c|c|c|c|c|}
\hline RE5P & 1 & 2 & 3 & 4 & 5 & 10 & 10 & 30 & 40 \\
\hline J & 1.00 & & & & & & & INTE P & \\
\hline 2 & 0.20 & 1.00 & & & & & & rave & \\
\hline 3 & 0.75 & -0.45 & 1.00 & & & & & & \\
\hline 4 & 0.01 & -0.32 & 0.34 & 1.00 & & & & & \\
\hline 5 & 0.34 & 0.35 & 0.58 & -0.13 & 1.00 & & & & \\
\hline 10 & $0.8 \mathrm{~B}$ & 0.54 & 0.36 & -0.23 & 0.33 & 1.00 & & & \\
\hline 20 & 0.39 & 0.76 & -0.20 & -0.51 & 0.60 & 0.70 & 1.00 & & \\
\hline 30. & $=0.48$ & -0.52 & 0.02 & 0.25 & -0.58 & $8-0.70$ & -0.87 & 1.00 & \\
\hline 40 & 0.38 & 0.83 & -0.27 & -0.40 & 0.63 & 0.71 & 0.93 & -2.71 & 1.10 \\
\hline
\end{tabular}

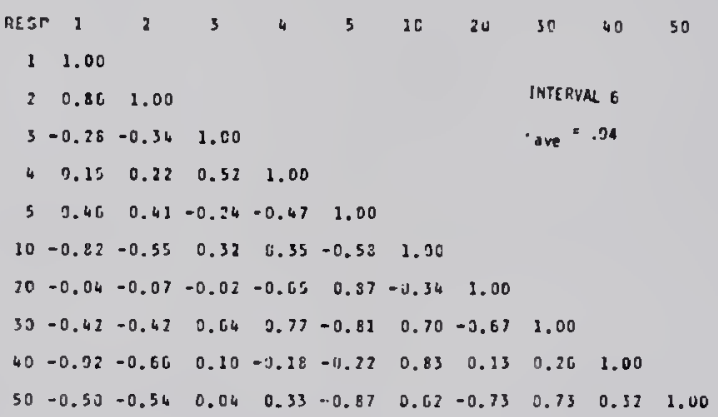

TABLES B. 1 through B.7

Response Correlation Coefficients The above tables provide the correlation coefficients between the ten response waveforms in Figure 3.2 for each $135 \mathrm{msec}$ interval. 

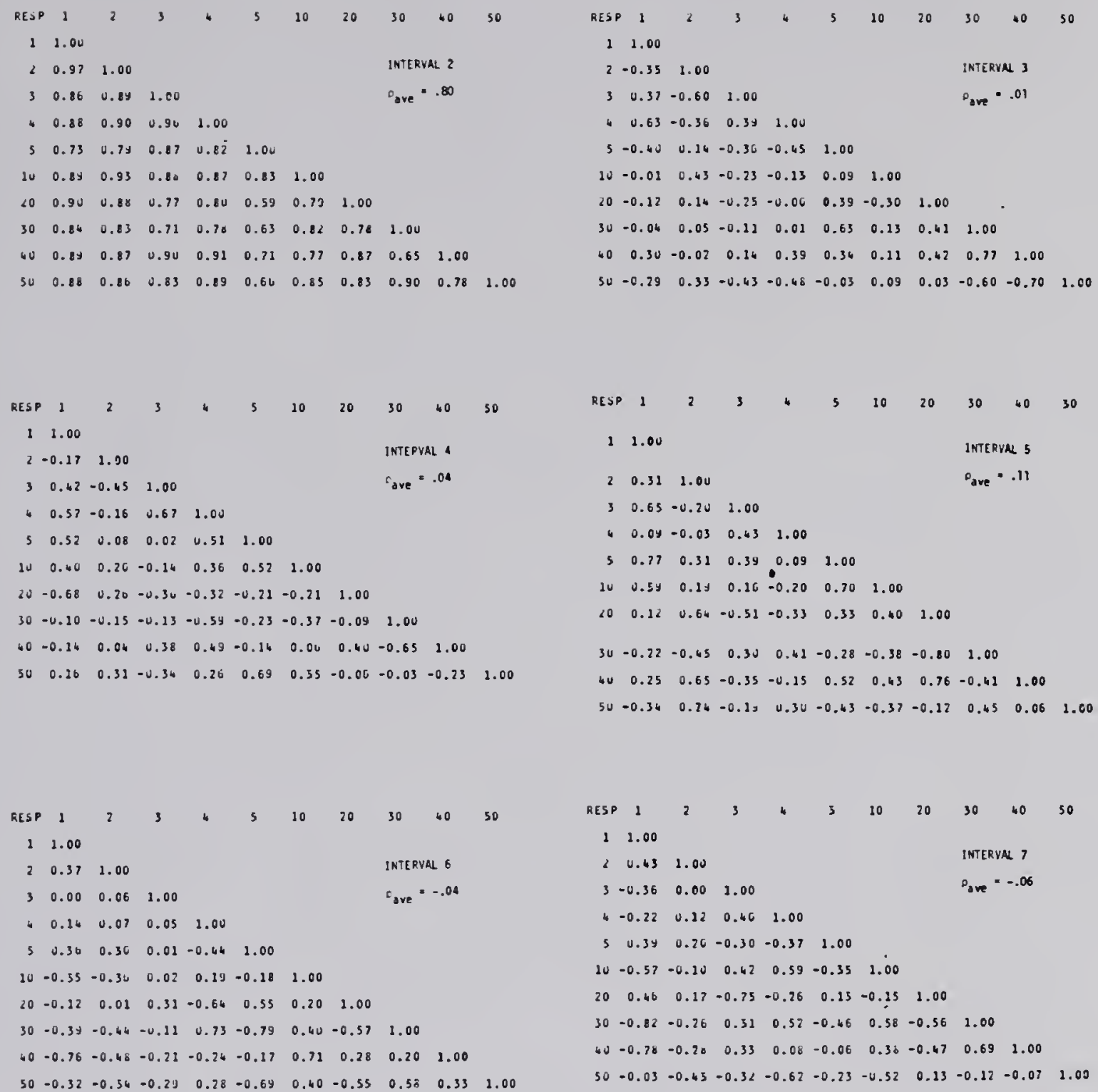

TABLES B. 8 through B. 13

EER Correlation Coefficients for the 0.3 to $50 \mathrm{~Hz}$ Bandwidth

The above tables provide the correlation coefficients between the ten wide band response waveforms in Figure B.1. These response waveforms were shifted to compensate for phase differences introduced by the filtering process. The above intervals therefore correspond to those segmented to narrow-band EER's. 


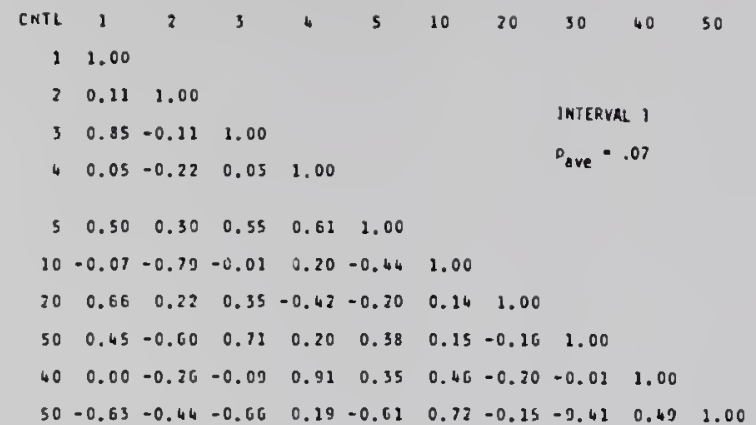

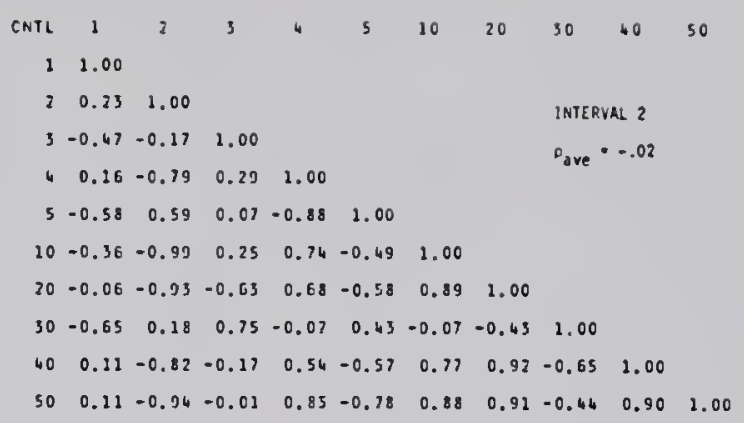

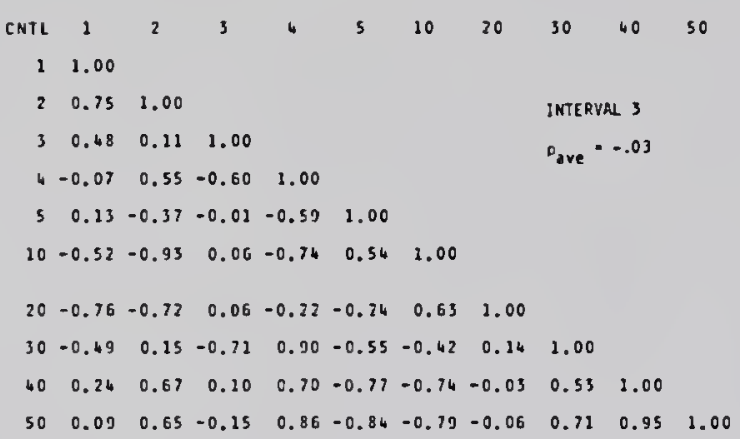

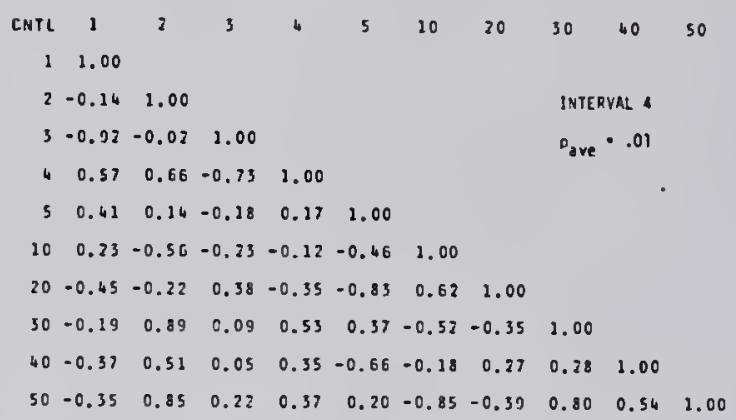

\section{Dave $\cdot 01$}

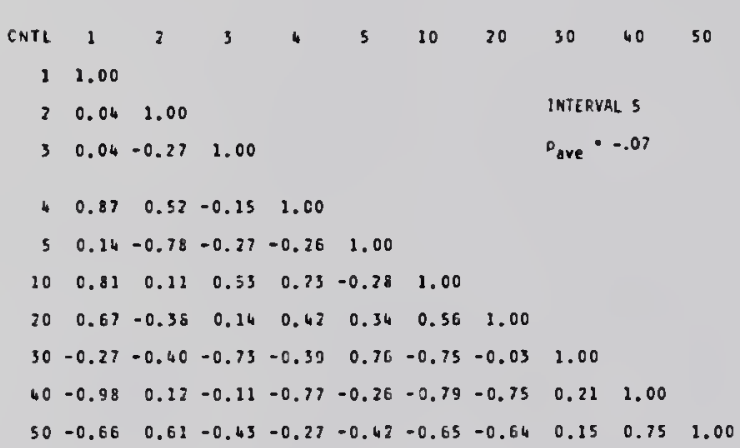

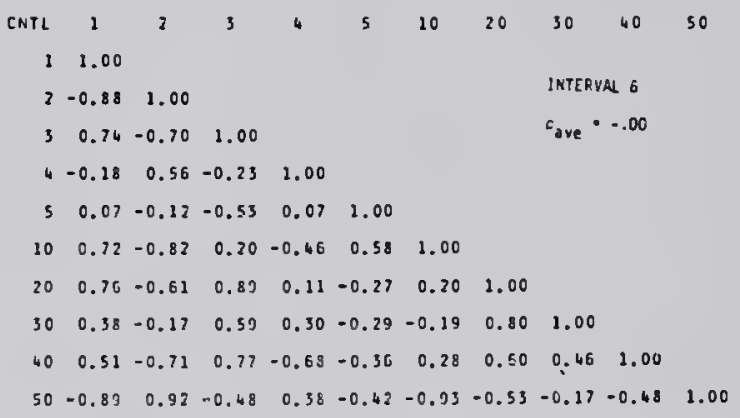

TABLES B. 14 through B. 20 Control Correlation Coefficients The above tables provide the correlation coefficients between the ten control waveforms in Figure 3.4 for each $135 \mathrm{msec}$ interval. 
$\tau$

\begin{tabular}{|c|c|c|c|c|c|c|c|c|c|c|}
\hline & .00 & .05 & .10 & .15 & .20 & .25 & .30 & .35 & .40 & .45 \\
\hline .00 & 1.00 & & & & & & & & & \\
\hline .05 & 0.90 & 0.41 & & & & & & & & \\
\hline .10 & 0.88 & 0.53 & 0.52 & & & & & & & \\
\hline .15 & 0.79 & 0.30 & 0.28 & 0.22 & & & & & & \\
\hline .20 & 0.71 & 0.10 & 0.35 & 0.27 & 0.28 & & & & & \\
\hline .25 & 0.86 & -0.03 & 0.17 & 0.03 & 0.09 & 0.11 & & & & \\
\hline .30 & 1.07 & 0.04 & 0.38 & -0.07 & -0.06 & -0.09 & 0.00 & & & \\
\hline .35 & 0.58 & 0.10 & 0.34 & -0.06 & -0.07 & -0.06 & -0.09 & 0.00 & & \\
\hline .40 & 0.63 & 0.07 & 0.21 & -0.17 & 0.10 & -0.12 & -0.04 & -0.08 & 0.16 & \\
\hline .45 & 0.57 & 0.20 & 0.06 & 0.00 & 0.06 & -0.01 & -0.08 & 0.01 & -0.13 & 0.15 \\
\hline .50 & 0.51 & 0.08 & -0.01 & 0.01 & -0.21 & 0.12 & -0.10 & 0.00 & -0.19 & -0.05 \\
\hline .55 & 0.62 & 0.13 & 0.22 & -0.02 & 0.06 & -0.16 & -0.00 & -0.24 & -0.27 & -0.23 \\
\hline .60 & 0.89 & 0.20 & 0.32 & -0.09 & 0.05 & -0.02 & -0.23 & -0.28 & -0.28 & -0.35 \\
\hline .65 & 0.53 & 0.27 & 0.12 & -0.04 & -0.12 & -0.08 & 0.05 & -0.14 & -0.17 & -0.24 \\
\hline .70 & 0.73 & 0.25 & 0.18 & 0.05 & -0.02 & -0.16 & -0.09 & -0.09 & -0.29 & -0.32 \\
\hline .75 & 0.77 & 0.30 & 0.13 & -0.01 & 0.02 & -0.13 & -0.16 & -0.14 & -0.15 & -0.30 \\
\hline .80 & 0.59 & 0.26 & 0.28 & 0.12 & 0.03 & -0.02 & -0.05 & -0.21 & -0.14 & -0.16 \\
\hline .85 & 0.57 & 0.20 & 0.18 & 0.18 & 0.02 & -0.07 & -0.10 & -0.19 & -0.12 & -0.12 \\
\hline .90 & 0.79 & 0.36 & 0.14 & -0.01 & 0.08 & -0.01 & -0.01 & -0.09 & -0.03 & -0.13 \\
\hline
\end{tabular}

TABLE B. 21

Values for $\tilde{C}_{f}(t, t-\tau)$ in Figure 4.1

The following values are measured relative to $\tilde{\mathrm{C}}_{f}(0,0)$ and the values of $t$ and $\tau$ are given in seconds. 


\section{REFERENCES}

Mmassian, V.E.,; Waller, H.J., and Macy, J., Jr.: Neural mechanisms of the primary somatosensory evoked potential. Ann $N$ Acad Sci, $112: 5-32,1964$.

Andersen, P., and Andersson, S.: Physiological Basis of Alpha Rhythm. New York, Appleton-Century-Crofts, 1968, $263 \mathrm{pp}$.

Barlow, J.S.: An electronic method for detecting evoked responses of the brain and for reproducing their average waveforms. Electroenceph C1in Neurophysiol, 9:340-343, 1957.

Barlow, J.S.: Autocorrelation and crosscorrelation analysis in electroencephalography. IRE Trans Med Electron, ME-6:179-183, 1959.

Barlow, J.S.: Rhythmic activity induced by photic stimulation in relation to intrinsic alpha activity of the brain in man. Electroenceph Clin Neurophysiol, 12:317-326, 1960.

Barlow, J.S.: Electronic simulation as an aid in evaluating conputeranalyzed EEG data. Electroenceph Clin Neurophysiol, 22:381-386, 1967.

Bendat, J.S.: Mathematical analysis of average response values for nonstationary data. IEEE Trans Biomed Engng, BME-11:72-81, 1964.

Bendat, J.S., and Piersol, A.G.: Measurement and Analysis of Random Data. New York, Wiley, $1966,3 \overline{90} \mathrm{pp}$.

Bergamini, L., and Berganasco, B.: Cortical Evoked Potentials in Man. Springfield, Thomas, $1967,116 \mathrm{pp}$.

Berkhout, J., and Walter, D.0.: Temporal stability and individual differences in the human EEG: an analysis of variance of spectral values. IEEE Trans Biomed Engng, BNE-15:165-168, 1968.

Bishop, G.H., ard Clare, M.H.: Relations between specifically evoked and "spontaneous" activity of the optic cortex. Electroenceph Clin Neurophysiol, 5:321-330, 1953.

Bogacz, J.; Varzulli, A.; Handler, P., and Garcia-Austt, E.: Evoked responses in main. II. Habituation of visual evoked response. Acta Neurol Lat Amer, 6:353-362, 1960. 
Brazier, M.A.B.; Cobb, W.A.; Fischgold, H.; Gastaut, H. ; Gloor, P.; Hess, R.; Jasper, H.; Leob, C. ; Magnus, O.; Dampiglione, G.; Remond, A.; Stom van Leeuwen, W., and Walter, W. Grey: PreIiminary proposal for an EEG teminology by the teminology committee of the International Federation for Electroencephalography and Clinical Neurophysiology. Electroenceph Clin Neurophysiol, 13:646-650, 1961 .

Brazier, M.A.B.: Varieties of computer analysis of electrophysiological potentials. In Cobb, W., and Norocutti, C. (Eds.): The Evoked Potentials. (Electroenceph Clin Neurophysio1, supp1. 26.) Amsterdam, Elsevier, 1967, pp. 1-8.

Campbe11, J.; Bower, E.; Dwyer, S.J., and Lago, E.G.: On the sufficiency of autocorrelation functions as EEG descriptors. IEEE Trans Biomed Engng, BME-14:49-52, 1967.

Childers, D.G.; Perry, N.W. , Jr., and Dawson, W.W.: Frequency filtering and computer summation analysis of visual evoked retinal and cortical potentials in noise. In Enslein, K. (Ed.) : Data Acquisition and Processing in Biology and Medicine. New York, Pergamon, 1968, $\overline{\text { vol. }}$ 5, pp. $39 \overline{-61 .}$

Childers, D.G., and Perry, N.W., Jr.: Alpha-like activity in vision. submitted paper, 1969.

Cobb, W.A.: The normal adult EEG. In Hill, D., and Parr, G. (Eds.) : Electroencephalography. A Symposium on its Various Aspects. London, llacdonald, 1963, pp. 232-249.

Cooper, R.; Winter, A.L.; Crow, H.J., and Walter, W.G.: Comparison of subcortical, cortical and scalp activity using chronically indwelling electrodes in man. Electroenceph Clin Neurophysiol, $18: 217-218,1965$.

Creutzfeldt, O.D.; Watanabe, S., and Lux, H.D.: Relations between EEG phenomena and potentials of single cortical cells. I. Evoked responses after thalmic and epicortical stimulation. II. Spontaneous and convulsoid activity. Electroenceph Clin Neurophysiol, $20: 1-37,1966$.

Creutzfeldt, O.D., and kuhnt, U.: The visual evoked potential: physiological, developmental, and clinical aspects. In Cobb, W., and Morocutti, C. (Eds.): The Evoked Potentials. (Electroenceph Clin Neuropiysiol, suppl. 26.) Amsterdam, E1sevier, 1967, pp. 29 41.

Davenport, Wilbur B., Jr., and Root, William L. : An Introduction to the Theory of Random Signals and Noise. New York, NcGraw-Hil1, $195 \overline{8}$ $393 \mathrm{pp}$. 
Dawson, G.D.: A sumnation technique for the detection of small evoked potentials. Electroenceph Clin Neurophysiol, 6:65-84, 1954.

Dawson, W.W. ; Stewart, H.L. ; Perry, N.W., Jr., and Childers, D.G.: Pooling of human visual evoked signals: a demonstration of components above $100 \mathrm{~Hz}$. Nature, 220:980-984, 1968.

De Lucchi, M.R.; Garoutte, B., and Aird, R.B.: The scalp as an electroencephalographic averager. Electroenceph Clin Neurophysiol, $14: 191-196,1962$.

Donchin, E.: A multivariate approach to the analysis of average evoked potentials. IEEE Trans Biomed Engng, BME-13:131-139, 1966.

Dustman, R.E., and Beck, E.C.: The visually evoked potentials in twins. Electroenceph Clin Neurophysiol, 19:570-575, 1965.

Eccles, J.C.: Cerebral synaptic mechanisms. In Eccles, J.C. (Ed.): Brain and Concious Experience. New York, Springer-Verlag, 1966 pp. 24-58.

Elul, R.: Statistical mechanisms in generation of the EEG. In Fogel, L.F., and George, F.W. (Eds.): Progress in Biomedical Engineering (San Diego Symposium). New York, Spartan, 1967, vol. 1, pp. 131150 .

Elul, R.: Brain waves: intracellular recording and statistical analysis help clarify their physiological significance. In Enslein, K. (Ed.): Data Acquisition and Processing in Biology and Medicine. New York, Pergamon, 1968, vol. 5, pp. 93-117.

Galambos, R., and Davis, H.: The response of sing1e auditory-nerve fibers to acoustic stimulation. J Neurophysiol, 15:281-286, 1943.

Garcia-Austt, E.; Vanzulli, A.; Bogacz, J., and Rodriguez-Barrios, R.: Influence of the ocular muscles upon photic habituation in man. Electroenceph Clin Neurophysiol, 15:281-286, 1963.

Goldstein, M.H., and Weiss, T.F.: Mathematical Statistics. (Appendix B) In Rosenblith, W.A. (Ed.): Processing Neuroelectric Data. Cambridge, M.I.T., 1962, pp. 85-97.

Haider, M.: Spong, P., and Lindsley, D.B.: Attention, vigilance, and cortical evoked potentials in humans. Science, 145:180-182, 1964.

Harris, E.K., and Woody, C.D.: Use of an adaptive filter to characterize signal-noise relationships. Computer and Biomed Res, $2: 242-273,1969$. 
Humphrey, Donald R.: Re-analysis of the antidromic cortical response. II. On the contribution of cell discharges and psps to the evoked potentials. Electroenceph Clin Neurophysiol, 25:421-442, 1968.

John, E.R.; Ruchkin, D.S., and Villegas, J.: Experimental background: signal analysis and behavioral correlates of evoked potential configurations in cats. Ann $\underline{N Y}$ Acad Sci, 112:362-420, 1964.

Johnson, L.C., and Ulett, G.A.: Quantitative study of pattern and stability of resting electroencephalographic activity in a young adult group. Electroenceph Clin Neurophysiol, 11:233$249,1959$.

Kitasato, H., and Hatsuda, T.: The degree of contamination of averaged response with alpha waves and alpha-like waves evoked by photic stimulation. Jap J Physiol, 15:492-504, 1965.

Kitasato, H.: The relation between the photic driving of EEG and the response evoked by photic stimulation in man. Jap J Physiol, $16: 238-253,1966$.

Kooi, K.A., and Bagchi, B.K.: Visual evoked responses in man: normative data. Ann NY Acad Sci, 112:254-269, 1964.

Lilliefors, H.W.: On the Kolmogorov-Smirnov test for nomality with mean and variance unknown. J A S A, 62:389-402, 1967.

Miller, Inwin, and Freund, John E.: Probability and Statistics for Engineers. Englewood Cliffs, Prentice-Ha11, $1965,432 \mathrm{pp}$.

Negin, M.: Detection of Aperiodic Activity in the Electroencephalogram. doctoral dissertation, U. of Fla., 1968.

Perry, N.W., Jr., and Copenhaver, R.S.: Differential cortical habituation with stimulation of central and peripheral retina. Percept Motor Ski11s, 20:1209-1213, 1965.

Perry, N.W., Jr.: Signal versus noise in the evoked potential. Science, 153:1022, 1966.

Perry, N.W., Jr.: Childers, D.G., and McCoy, J.G.: Binocular addition of the visual evoked response at different cortical locations. Vision Res, 8:567-573, 1968.

Perry, N.W., Jr., and Childers, D.G.: The Human Visual Evoked Response. Springfield, Thomas, 1969, 134 pp.

Pegan, D.: An effect of stimulus colour on average steady-state potentials evoked in man. Nature (London), 210:1056-1057, 1966a. 
Regan, D.: Some characteristics of average steady-state and transient responses evoked by modulated light. Electroenceph Clin Neurophysiol, 20:258-248, 1966b.

Rosenblith, W.A.: Some quantifiable aspects of the electrical activity of the nervous system (with emphasis upon responses to sensory stimuli). In Oncley, J.L. (Ed.): Biophysical Science - A Study Program. New York, Wiley, 1959, pp. 532-545.

Ruchkin, D.S.: An analysis of average response computations based upon aperiodic stimuli. IEEE Trans Biomed Engng, BM:E-12:87-94, 1965.

Ruchkin, D.S.: Analysis of nonhomogeneous sequences of evoked potentials. Exp Neuro1, 20:275-284, 1969.

Saunders, M.G.: Amplitude probability density studies on alpha and alpha-like patterns. Electroenceph C1in Neurophysiol, 15:761-767, 1963.

Schimel, H.: The \pm reference: accuracy of estimated mean component in average response studies. Science 157:92-94, 1967.

Underwood, Benton J.; Duncan, Carl P.; Taylor, Janet A., and Cotton, John W.: Elementary Statistics. New York, Appleton-Century-Crofts, 1954, 239 pp.

Walter, D.O.; Kado, R.T.; Rhodes, J.M., and Adey, W.R.: Electroencephalographic baselines in astronaut candidates estimated by computation and pattem recognition techniques. Ae rospace Med, 38:371-379, 1967.

Werre, P.F., and Smith, C.J.: Variability of responses evoked by flashes in man. Electroenceph Clin Neurophysiol, 17:644-652, 1964.

Woody, C.D.: Characterization of an adaptive filter for the analysis of variable latency neuroelectric signals. Med Biol Engng, 5:539$553,1967$.

Zerlin, S., and Davis, H.: The variability of single evoked vertex potentials in man. Electroenceph Clin Neurophysiol, 23:468-472, 1967. 


\section{BIOGRAPHICAL SKETCH}

Timothy C. Doyle was born April 29, 1943, at Ponca City, Oklahoma. In June, 1961, he was graduated from Coral Gables High School. In December, 1965, he received the degree of Bachelor of Science in Electrical Engineering from the University of Florida. Following his graduation, he worked as a graduate assistant in the Department of Electrical Engineering until August, 1966. From September, 1966, until the present time he has worked as a research assistant to the Visual Sciences Laboratory at the University of Florida. He received his Master of Science in Electrical Engineering in April, 1967, and since that time has pursued his work toward the Doctor of Philosophy. He was employed as a teaching associate in the summer of 1969 .

Timothy C. Doyle is married to the former Susan Foxworth. $\mathrm{He}$ is a menber of Eta Kappa $\mathrm{Nu}$ and an associate member of Sigma Xi. 
This dissertation was prepared under the direction of the chairman of the candidate's supervisory committee and has been approved by all members of that comnittee. It was submitted to the Dean of the College of Engineering and to the Graduate Council, and was approved as partial fulfillment of the requirements for the degree of Doctor of Philosophy.

August, 1969

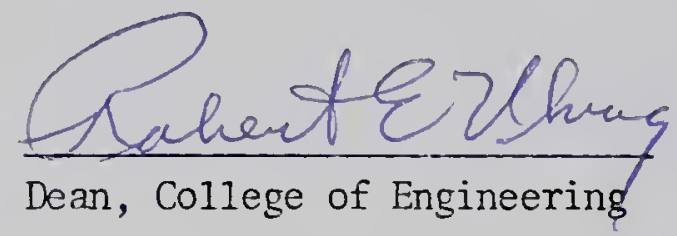

Dean, Graduate School

Supervisory Conmittee:

\section{Q.7. Ghelders}

Chairnan

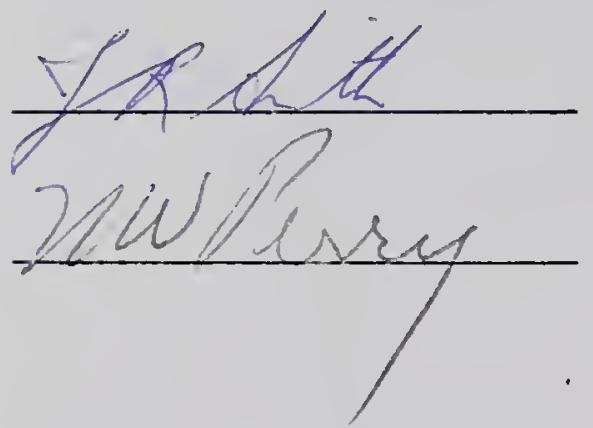


